$L \Lambda--10170-P R$

DE85 004488

\title{
Safeguards and Security Progress Report January-December 1983
}

Compiled by

Darryl B. Smith

\author{
DISCLAIMER
}

This report was prepared as an account of work sponsored by an agency of the United States Government. Neither the United States Government nor any agency thereof, nor any of their employees, makes any warranty, express or implied, or assumes any legal liability or responsibility for the accuracy, completeness, or usefulness of any information, apparatus, product, or process disclosed, or represents that its use would not infringe privately ownot rights. Reference herein to any specific commercial product, process, or service by trade name, trademark, manufacturer, or otherwise does not necessarily constitute or imply its endorsement, recommendation, or favoring by the United States Government or any agency thereof. The views and opinions of authors expressed herein do not necessarily state or reflect those of the United States Government or any agency thereof. 


\section{CONTENTS}

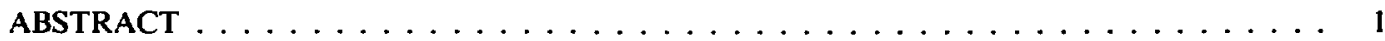

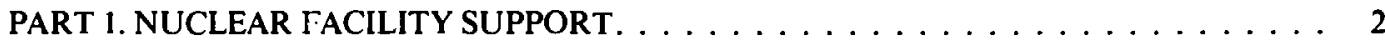

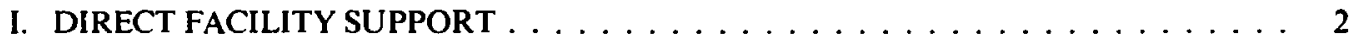

A. Albuquerque/Los Alamos National Laboratory . . . . . . . . . . . . . . . . 2

B. Idaho/Idaho National Engincering Laboratory (INEL) . . . . . . . . . . . . . . . 4

C. Idaho/Three-Mile Island (TMI) Nuclear Power Plant Unit 2 (TMI-2) . . . . . . . . . . 6

D. Nevada/Nevada Test Site (NTS) . . . . . . . . . . . . . . . . . . . . 6

E. Oak Ridge/Y-12 Facility-Automated NDA Instruments for In-Line High-Enriched Uranium (HEU) Accounting. . . . . . . . . . . . . . . . 8

F. Oak Ridge/Censolidated Fuel Reprocessing Program (CFRP). . . . . . . . . . . 12

G. Oak Ridge/Portsmouth Gaseous Diffusion Plant (GDP) . . . . . . . . . . . . . 13

H. Oak Ridge/Portsmouth Gas Centrifuge Enrichment Plant (GCEP) . . . . . . . . . . 13

I. Richland/Fuels and Materials Examination Facility (FMEF) . . . . . . . . . . . 14

J. Richland/Hanford PUREX: Screw Calciner Holdup Monitor . . . . . . . . . . . . . 16

K. Savannah River/Naval Fuel Materials Facility (FMF) . . . . . . . . . . . . . . . 17

L. Savannah River/New Special Recovery (NSR) Facility . . . . . . . . . . . . . 18

M. Savannah River/FB Line . . . . . . . . . . . . . . . . . . . 20

N. Ad Hoc Field Assistance/Savannah River Laboratory (SRL) . . . . . . . . . . . . 21

II. SAFEGUARDS TECHNOLOGY TRAINING $\ldots \ldots \ldots \ldots \ldots \ldots \ldots$

PART 2. SECURITY DEVELOPMENT AND SUPPORT . . . . . . . . . . . . 25

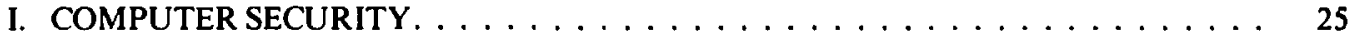

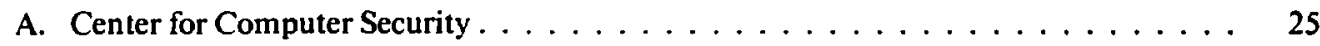

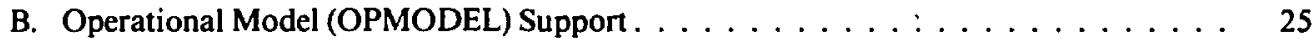

C. Secure Computer Systems . . . . . . . . . . . . . . . . . . . 26

D. Computer and Network Access Controls. . . . . . . . . . . . . . . 26

E. Management Support Tools . . . . . . . . . . . . . . . . . . . 27

II. OPERATIONAL SECURITY (OPSEC) AND VULNERABILITY

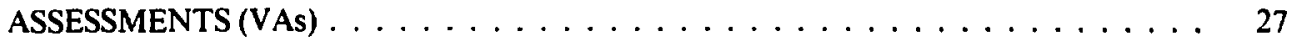

PART 3. SAFEGUARDS TECHNOLOGY DEVELOPMENT. . . . . . . . . . . . . 29

I. EQUIPMENT DEVELOPMENT AND ENGINEERING . . . . . . . . . . . . . 29

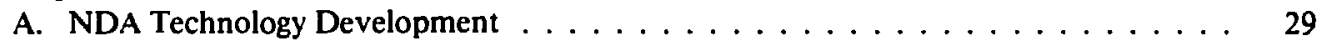

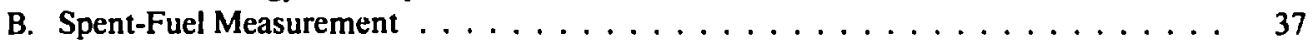

C. Containment and Surveillance Technology Development . . . . . . . . . . . 39

D. Chemical and Isotopic Analysis . . . . . . . . . . . . . . . . . 42

E. Standards Development . . . . . . . . . . . . . . . . . . . . 45 
II. MATERIALS CONTROL AND ACCOUNTING (MC\&A) TECHNOLOGY AND APPLICATIONS DEVELOPMENT . . . . . . . . . . . . . . . 46

A. Design and Evaluation Methods for an integrated Safeguards System . . . . . . . . 46

B. MC\&A Technology Development . . . . . . . . . . . . . . 48

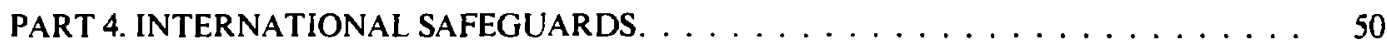

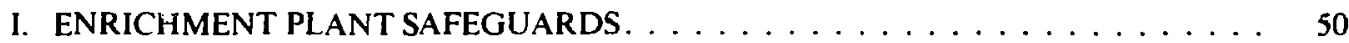

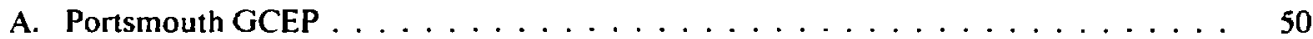

B. Compact ${ }^{252} \mathrm{Cf}$ Shuffler for $\mathrm{UF}_{6}$ Measurements $\ldots \ldots \ldots \ldots \ldots \ldots$

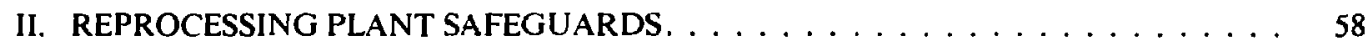

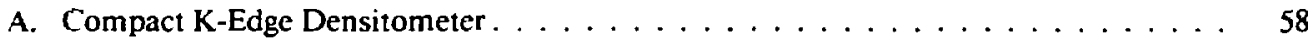

B. Dounreay Shuffer . . . . . . . . . . . . . . . . . . . . . . . 59

III. CONTAINMENT AND SUR VEILLANCE FOR INTERNATIONAL SAFEGUARDS . . 60

A. The Interface Between Materials Accounting and Containment/Surveillance . . . . . 60

B. Optimizing the Design of International Safeguards Inspection Systems. . . . . . . . . 60

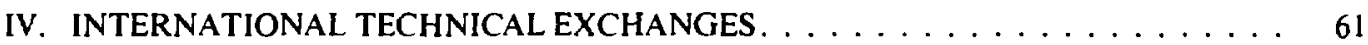
A. Program of Technical Assistance to IAEA Safeguards (POTAS) . . . . . . . . . . . 61
B. Technical Support to the Power Reactor and Nuclear Fuel Development Corporation (PNC) TASTEX Instruments . . . . . . . . . . . . . . . . 62
C. Joint Ispra $/$ Los Alamos Program . . . . . . . . . . . . . . . . . . 62

V. NUCLEAR NONPROLIFERATION ACT (NNPA) COURSE IN NUCLEAR

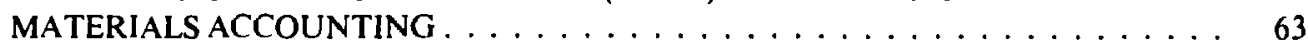

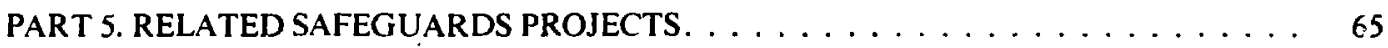

1. ESTIMATION METHODS FOR THE PROCESS HOLDUP OF SPECIAL NUCLEAR MATERIALS . . . . . . . . . . . . . . . 65

II. HANDBOOK OF NUCLEAR SAFEGUARDS MEASUREMENT METHODS . . . . . 65

III. SECONDARY REFERENCE MATERIALS FOR THE NRC. . . . . . . . . 65

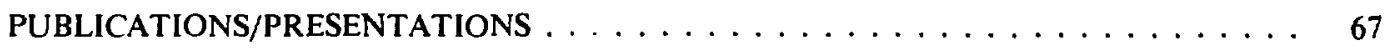

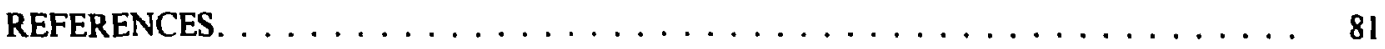




\title{
SAFEGUARDS AND SECURITY PROGRESS REPORT
}

\author{
January—December 1983 \\ Compiled by \\ Darryi B. Smith
}

\begin{abstract}
From January to December 1983, the Los Alamos Safeguards and Security Program was involved in the activities described in the first four parts of th : report: Nuclear Facility Support, Security Development and Support, Safeguards Technology Development, and International Safeguards. Part 1 covers efforts of direct assistance to the Department of Energy (DOE) and Nuclear Regulatory Commission (NRC) licensee facilities. This assistance includes consultation on materials accounting problems, development of specialized techniques and instruments, and comprehensive participation in the design and implementation of advanced safeguards systems. In addition, a series of training courses in various aspects of safeguards makes the technology more accessible to those who must apply it. Part 2 treats activities aimed at the security of information and computer systems. Our focus this period was on continuing the activities of the Computer Security Center, which provides the basis for encouraging and disseminating this emerging technology, and on the development and demonstration of secure computer systems. Fart 3 describes the broad development efforts essential to continuing improvements in the practice of safeguards. Although these projects are properly classified as developmental, they address recognized problems that commonly occur in operating facilities. Finally, Part 4 covers international safeguards activities, including both support to the International Atomic Energy Agency and bilateral exchanges. Enrichment plant safeguards, especially those concerning the Gas Centrifuge Enrichment Plant, required a significant portion of our resources. These efforts are beginning to provide substantial returns on our investment in technology transfer, not only in raising the level of safeguards effectiveness but also in our benefiting from field experiences in operating environments.

While most projects described in this report were sponsored by the DOE, Office of Safeguards and Security, safeguards-related activities that have other sponsors are reported in Part 5. The final part of this report gives titles and abstracts of Los Alamos safeguards research and development reports, technical journal articies, and conference papers that were published in 198 ?
\end{abstract}




\section{PART 1: NUCLEAR FACILITY SLPPORT}

\section{DIRECT FACILITY SUPPORT}

\section{A. Albuquerque/Los Alamos National Laboratory}

1. Nuclear Materials Storage Facility (C. Coulter, J. Markin, and $W$. Whitty, $Q-4)$. A new centralized storage facility (CSF) is to be built at the Los Alamos Plutonium Processing Facility to provide long-term storage for nuclear materials and to serve as a central shippingreceiving point for these materials. Development of the CSF is in an early design phase, and many of the physical and operational features of the building are still fluid or undetermined. This provides an excellent opportunity to examine and, if necessary, modify the proposed safeguards system while a certain amount of design flexibility still exists. In a previous study (see Part 3, Sec. II.A), we developed a methodology for the design and evaluation of integrated nuclear safeguards systems and are applying this methodology to that part of the safeguards design for the CSF that has already been defined to evaluate its probable performance with respect to the current Department of Energy/Office of Safeguards and Security (DOE/OSS) threat guidance.

Subjects to which we have given particular attention include the relationship of the CSF to the protected area in which it will be located, protective fences and exterior intrusion detection systems for the building, potential personnel concealment areas in the interior of the vault building, operational efficiency of the interior design, use of security inspectors in the building, locations of portal monitors for nuc!ear materials, interior intrusion-detection systems, protection of the heating, ventilating, and air conditioning (HVAC) system, materials measurement and accounting procedures, locations of offices for personnel, duress alarm systems, and emergency response systems.

Based on the results of the study, we prepared a draft report offering a number of comments and suggestions concerning the CSF design. We concurred with the proposal to use a drive-through truck bay for the building and suggested a possible vault-building physical design with a truck bay of this type. This design provides an efficient traffic-flow pattern for materials transfers, provides the vault supervisor with a clear view of most interior areas of the building, and places all but two offices on an exterior wall a maximum distance from radioactive materials. We also made recommendations concerning the choice of interior intrusion-detection devices and the placement of duress alarms, the use of security inspectors in the building, and the use of portal radiation monitors. We suggested an alternative location for the materials measurement area, and did shielding calculations to verify the practicality of the alternative site. Recommendations were made about safeguards procedures related to the vault door locks, use of the elevator for materials movement, and entry of materials transactions on the materials accounting computer terminal. Emergency response measures for forcible attack were suggested. We concurred with plans for future automation of a portion of the vault and pointed out additional safeguards benefits to be derived from such an automated system. Finally. we recummended methods for protecting the HVAC ductwork.

It is important that the CSF safeguards design be monitored throughout the remainder of the design and implementation process and updated as required. In addition, an in-depth evaluation of the computerized materials accounting system should be performed.

2. Measurement Control Program (MCP) for Balances (A. Goldman, Q-4). The DOE Order 5630.2 for Materials Control and Accounting (MC\&A) requires, where practical, an MCP to ensure that "all scales and balances are maintained in good working condition and calibrated pursuant to an established control program." We have completed a siudy that surveys the current MCP for balances at the Los Alamos Plutonium Processing Facility and that recommends a revised MCP. The study was based on 2 years of accumulated data from daily weighings of 1 - and 4-kg standards on 25 commercial electronic balances ( $5-\mathrm{kg}$ capacity and $0.1-\mathrm{g}$ read-out). The following are principal findings and recommendations.

- Individual instrument biases range between $-0.15 \mathrm{~g}$ and $+0.10 \mathrm{~g}$ with most negative biases attributable to a truncation of readout digits to tenths of a gram. We recommended that bias be computed as the average difference between the measured and reference values of standard weights.

- Standard deviations calculated for individual balances for the $1-\mathrm{kg}$ weights $(0.04 \mathrm{~g}$ to $0.09 \mathrm{~g})$ and the $4-\mathrm{kg}$ weights $(0.07 \mathrm{~g}$ to $0.13 \mathrm{~g})$ differ significantly from the common value $(0.15 \mathrm{~g})$ currently used in the accuracy test for all balances. We recommended an accuracy test be performed for each instrument by computing $Z$, the difference between the observed and standard weight divided by $\hat{\text {, }}$ 
where ô is the average standard deviation computed over previous recalibrations. if $|Z|$ is less than or equal to 1.96 , the accuracy test is passed. The standard is remeasured if $|\mathrm{Z}|$ is greater than 1.96 or less than 2.98 , and the balance is recalibrated if $|Z|$ is greater than or equal to 2.98 .

- Five repeated weighings in a short period of time are not a useful check on precision and frequently resulted in standard deviations equal to zero; that is, all five readings were identical. We recommended a precision test be performed daily for each instrument by computing $U$, the ratio of the variance of the five most recent standard measurements divided by $\hat{o}^{2}$. The test is passed if $U$ is less than or equal to 2.37 , is repeated if $U$ is between 2.37 and 3.32, and is failed if $U$ is greater than or equal to 3.32. The instrument is recalibrated if it fails the test.

- The only current check for randomness involves a subjective review of monthly control charts. Although this review is useful, an objective test that uses data from overlapping months is better. We recommended a randomness test that uses the mean square successive difference test be performed for each instrument using data from 20 successive day-to-day standard measurements.

- Defective balances might have been detected earlier if more attention had been paid to the number of recalibrations and the number of days between recalibrations. For example, one balance was removed only after it had been recalibrated 21 times in 201 working days. We recommend a balance be considered for removal if the average number of days between recalibrations is fewer than 20 . This test detects any unusual oscillations, and the balance is considered for repair if it fails the test.

\section{Improved Attribute Sampling Plans for Materials} Accounting (A. Goldman, Q-4). The DOE Order 5630.2 requires periodic inventories of special nuclear materials (SNM). Implementing this order takes a substantial investment of personnel resources at the Plutonium Processing Facility, which lias a storage vault containing approximately seven thousand items. We are investigating v*ays to reduce the personnel time and radiation exposure for item inventories through improved sampling plans that maintain the effectiveness of current inventory procedures while requiring smaller sample sizes. Although this study addresses improved inventory procedures for Los Alamos, the innovations would be applicable to any DOE facility that stores large numbers of SNM items.
The current single-sampling plan is defined by an acceptance number $\mathrm{c}$, sample size $\mathrm{n}$, and lot size $\mathbf{N}$. A sample consisting of $\mathbf{n}$ items is taken from $\mathbf{N}$ items and the number of defects $d$ is noted. If $d$ is less than or equal to $c$, the lot of size $\mathrm{N}$ is accepted; otherwise, it is rejected. Appropriate values of $\mathrm{c}$ and $\mathrm{n}$ are selected to satisfy risk combination (AQL, $\alpha, \mathrm{RQL}, \boldsymbol{\beta}$ ), where

AQ:- is the acceptable quality level, the maximum acceptable fraction of defectives in an individual lot;

$\alpha$ is the probability of rejecting a lot of AQL quality, that is, a lot with $A Q L \times N$ defective items, and often is called the false-alarm probability;

$\mathrm{RQL}$ is the rejectable quality level, the minimum per cent defective in an individual lot that can be considered unacceptable, ani

$\beta \quad$ is the probability of accepting a lot of RQL quality, that is, a lot with $R Q L \times N$ defective items. Beta is also called the nondetection probability.

The proposed double-sampling plan has four possibilities for acceptance or rejection of a lot: (1) accoptance after the first sample, (2) rejection after the first sample, (3) acceptance after the second sample, and (4) rejection after the second sample. The need for a second sample arises when results on the first sample do not permit a decision that the lot is either acceptable or should be rejected.

A double-sampling plan is defined by acceptance numbers $c_{1}, c_{2}$, and $c_{3}$; sample sizes $n_{1}$ and $n_{2}$; and lot size $N$. A sample of $n_{1}$ items is taken from $N$ items, and the number $d_{1}$ of defects is noted. If $d_{1}$ is less than or equal to $c_{1}$, the lot is accepted; if $d_{1}$ is greater than or equal to $c_{2}$, the lot is rejected. If $d_{1}$ is between $c_{1}$ and $c_{2}$, a second sample of $n_{2}$ items is taken from $N-n_{1}$ items, and the number $d_{2}$ of defects is noted. If $d_{1}+d_{2}$ is less than or equal to $c_{3}$, the lot is accepted; if $d_{1}+d_{2}$ is greater than $c_{3}$, the lot is rejected.

There is a unique sampling plan and at least one double-sampling plan that will satisfy the conditions of a specified risk set. The double-sampling plan usually will require a smaller sample size. The decision as to which double-sampling plan is selected depends upon the desired criteria. Two reasonable criteria are to (1) select $n_{1}$ s: : lll relative to $n_{2}$ or (2) choose a minimal average sample size $n_{1}+n_{2}$. These two criteria ordinarily cannot be satisfied simultaneously.

As a result of this study, the plutonium facility is in the process of replacing single-sampling plans with 
double plans. We expect to publish tables of doublesampling plans and will investigate multiple and Bayesian sampling plans to further reduce sample sizes.

4. Evaluation of In-Line Nondestructive Assay (NDA) Systems (D. F. Bowersox, MST-11; W. E. Haag, C. L. Heinherg, H. C. Muffly, and J. D. Torres, MST-12; E. L. Adams, N. Ensslin, S. T. Huse, S. S. Johnson, J. L. Parker, and T. E. Sampson, Q-1; F. Hsue, OS-2). The Los Alamos Plutonium Processing Facility usually relies on the destructive assay of a sample to determine the quantity of plutonium in incoming material. Each container must be opened and sampled and the sample sent elsewhere for isotopic and chemical assay. The elapsed time between receipt of material and reporting of results is a major handicap in this method. In addition, there is no proof that the sample is representative and that adsorption of moisture in the sample has not altered the results. Because NDA techniques are rapid, relatively inexpensive, and eliminate potential sampling problems, we have, for several years, carried on a highly successful joint project between safeguards research and development (R\&D) personnel and plutonium-processing personnel to design, construct, evaluate, and implement NDA systems for characterizing solids and liquids containing plutonium. The effectiveness of the NDA approach is validated by conventional chemical methods.

We have found that the nondestructive assay of solids containing plutonium by gamma spectrometry plus calorimetry is a cost- and time-effective method for determining plutonium. ${ }^{1-4}$ The multiple detecto tor the plutonium isotopics (MUDPI) assay instrument has been thoroughly evaluated and is now in routine operation.

An L $\mathrm{x}$-ray instrument, the low-plutonium solution assay instrument (LOSAI), ${ }^{5}$ designed for determining plutonium in solutions containing less than $200-\mathrm{mg}$ $\mathrm{Pu} / \mathrm{\ell}$, consists of a sample chamber in the glove box and a Si(Li) detector, shielding, and associated electronics outside the glovebox. The americium is removed from solutions with expected high americium/plutonium ratios (greater than 3 ), and the plutonium-rich phase is counted. The instrument was placed in-line and evaluated with plutonium solutions. Measurement control, separation procedures, and the precision and accuracy of the results have been tested, and the instrument is now used routinely to determine whether solutions from ion-exchange and precipitation processes are recycled or discarded.

A solution neutron coincidence counter ${ }^{6}$ for the assay of plutonium solutions containing 2 to $20 \mathrm{~g} \mathrm{Pu} / \mathrm{l}$ is being evaluated. The stability and precision of the counter is better than one per cent, and results were reproducible. We are now determining the effect of fluoride concentration on the neutron count.

\section{B. Idaho/Idaho National Engineering Laboratory (INEL)}

1. Installation of the Fuel Storage (FAST) Facility Delayed-Neutron Interrogator (G. W. Eccleston, H. $\mathbf{O}$. Menlove, D. C. Garcia, G. Ortiz, and T. Van Lyssel, Q-1). We recently completed installation of a large, dual-assay shuffler in the new Fluorinel Dissolution and FAST facility at INEL. This facility will receive, store, and dissolve zirconium-clad fuels from government research reactors and from the US Navy's nuclear ship propulsion program. ${ }^{7}$ The shuffler will measure the ${ }^{235} \mathrm{U}$ content of irradiated, highly enriched uranium fuels and of waste solids resulting from fuel dissolution. ${ }^{8-10}$

Assay performance was tested before installation in the FAST facility. " Four waste canister standards were fabricated by Los Alamos using uranium supplied by the EXXON Nuclear Idaho Company (ENICO). These standards contain varying amounts of ${ }^{235} \mathrm{U}$ in an appropriate matrix. One canister was only partially filled to determine the effects of fill height on waste assays. The calibration obtained from the waste standards is shown in Fig. 1.

The shuffler includes electronic and mechanical components located throughout the facility (Fig. 2). The Delayed Neutron Interrogator (Fig. 3) is located in a shielded cubicle at the side of the fuel dissolver cell. It is

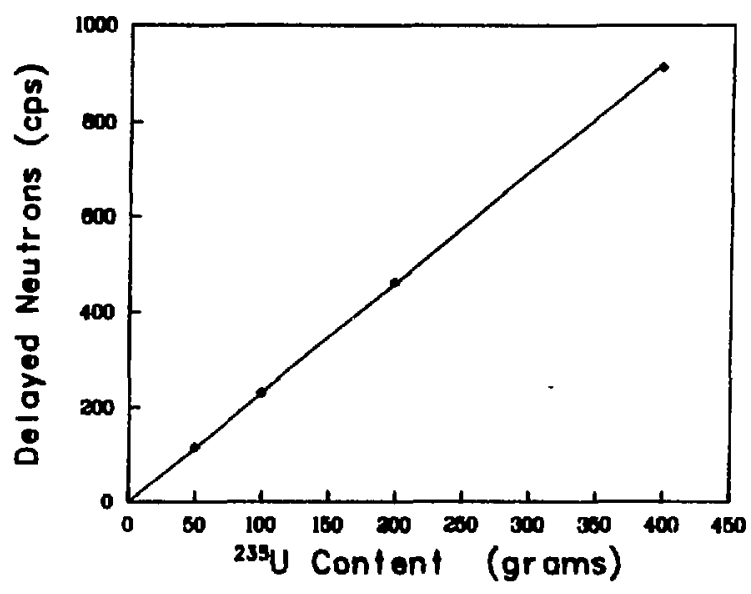

Fig. 1. Delayed-neutron detector response vs ${ }^{235} U$ mass for waste standards measured in the small-sample assay tube. 


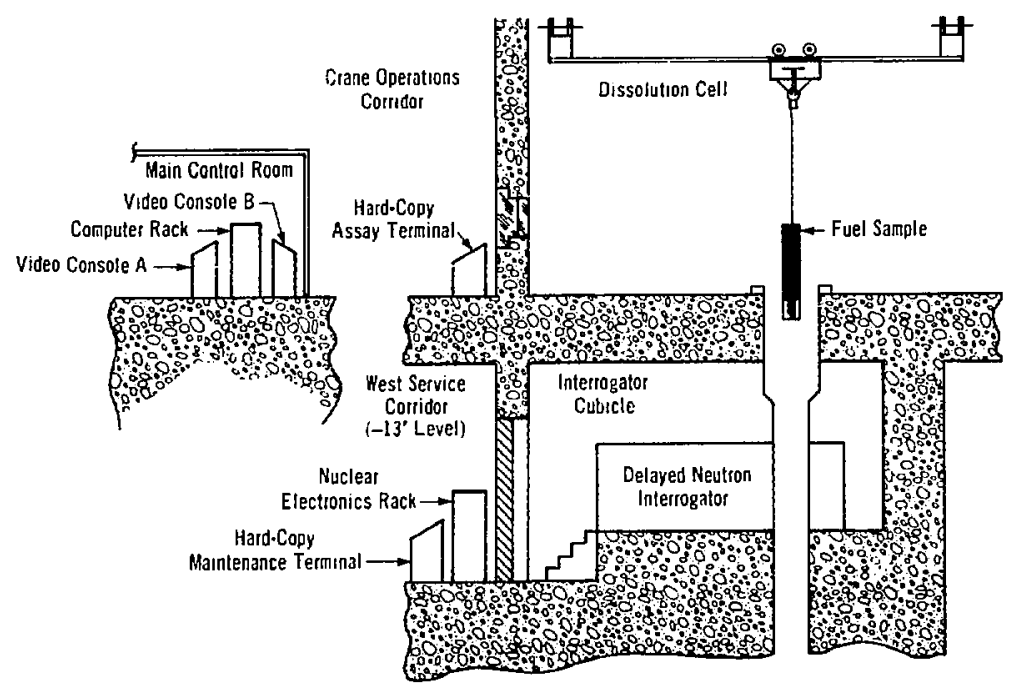

Fig. 2. System component locations in the FAST facility.

connected by cables to nuclear electronics mounted outside the cubicle. A computer in the operations room controls the instrument and receives measurement data from the nuclear electronics. The instrument control terminal is located adjacent to a hot-cell window, directly above the instrument cubicle. This location allows the operator to control the instrument while positioning samples visually in the assay tubes. A sample is measured while the dissolver-cell crane scans it through the appropriate measurement tube. The vertical position of the sample is determined by the crane con-

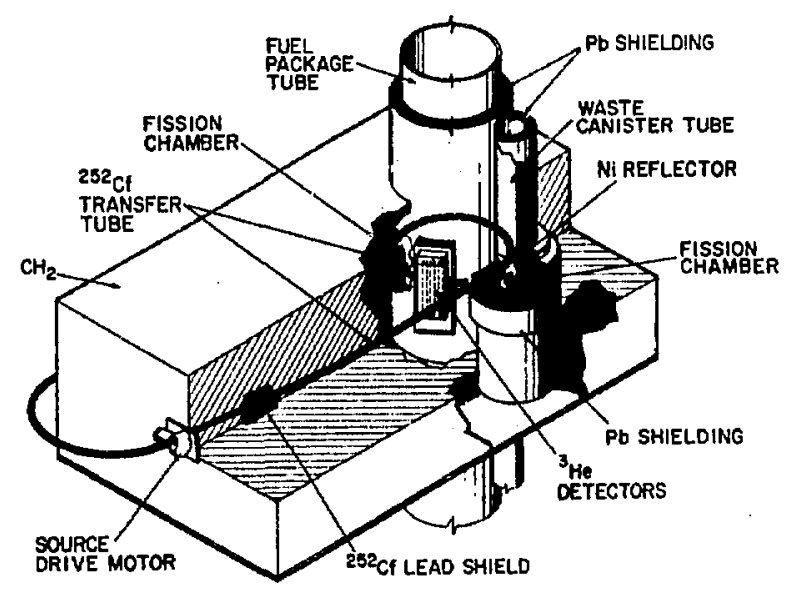

Fig. 3. Delayed Neutron Interrogator (length $6 \mathrm{~m}$ ) recently installed at the FAST facility. troller and the shuffler scan. Graphical displays of assay data are provided by color terminals located in the facility control room. These terminals also allow measurement data to be recalled for review from computer disk storage. The shuffler computer contains a communications link with the main facility computer.

Installation of the shuffler at the FAST facility was coordinated through the construction manager, Catalytic, Inc., and completed in 1983. All instrument parts are constructed and labeled to permit easy removal for maintenance. The modular design allowed the mechanical system to be installed in the facility in only a few weeks. The installation of the electronic control system was completed several months after the mechanical assembly when the required power, conduit, and cable runs were in place. Operational testing of the instrument followed installation, and software programs demonstrated the performance of the source transfer control system and other operating features of the instrument. The communications link between the shuffler computer and main facility computer was tested and functioned according to specifications. The crane's vertical position read-out is being installed by the vendor and will be tested when ready. In late 1984 a ${ }^{252} \mathrm{Cf}$ source will be installed in the Delayed Neutron Interrogator to demonstrate assay capability at Idaho.

ENICO is currently testing the shuffler's electrical system. The fuel storage area is scheduled to begin operation in late spring 1984, and the fuel dissolution area should begin operations in March 1985. The existing waste standards will be used in testing the performance of the shuffler in late 1984. Standards for fuel 
measurements will be developed concurrently with the first dissolution operations.

2. Idaho Fuel Processing Restoration Project (J. Barnes, R. Gutmacher, and D. Stirpe, Q-4). We reviewed the draft report $\mathrm{DOE} / \mathrm{ID} / 12235-7$, "Special $\mathrm{Nu}$ clear Materials Control and Accountability Study for Fuel Processing Restoration Project," prepared by GA Technologies, San Diego, California, under subcontract to the Ralph M. Parsons Company for the DOE Idaho Operations Office (July 1983). Our detailed comments addressed three major topics: (1) sampling and calibration of tanks; (2) materials balance areas, key measurement points, and applicable measurement methods; and (3) error propagation and materials balance calculations. Our response was sent to the Idaho Operations Office in August 1983.

C. Idaho/Three-Mile Island (TMI) Nuclear Power Plant Unit 2 (TMI-2) (J. K. Halbig, G. E. Bosler, J. R. Phillips, and P. M. Rinard, Q-1)

Los Alamos was asked by DOE/TMI to help measure the nuclear fuel material held up in some of the equipment contaminated during the TMI-2 reactor accident. We measured two demineralizer tanks that were on-line at various times during the incident. These tanks are in the cooling water make-up and purification system; they are $1.2 \mathrm{~m}$ in diameter and $2.1 \mathrm{~m}$ high. It was thought they contained up to $50,000 \mathrm{Ci}$ of radioactive material, although filters upstream were perforated, and it was uncertain how much fuel material had been deposited in the demineralizer tanks. The quantity of contained nuclear material had to be measured before the tanks could be emptied to avoid a possible criticality accident during cleanout.

The demineralizers were located in $4-$ by $4.3-\mathrm{m}$ cubicles that were not accessible to workers (dose rate approximately $3000 \mathrm{R} / \mathrm{h}$ ). Although the gamma-ray activity from fission products in the demineralizer was large, the neutron signal from the $\mathrm{e}$ spected fuel material was not measurable above backfiound.

The Hanford Engineering Development Laboratory (HEDL) prepared solid-state track recorders and a Compton recoil spectrometer to measure neutron and gamma-ray activity. To supplement their measurements, we used instrumentation developed for the spent-fuel measurement program: an ion chamber to measure total gamma-ray activity ( $10 \mathrm{~s} /$ data point) to infer the source distribution in the demineralizer and a
$\mathrm{Be}(\gamma, \mathrm{n})$ detector to measure high-energy gamma rays ( $900 \mathrm{~s} /$ data point) specific to a fission product, ${ }^{144} \mathrm{Pr}$, that could be directly linked to the fuel content. We calibrated the $\operatorname{Be}(\gamma, \mathrm{n})$ detector at Los Alamos using an 8Ci calibration source. Monte Carlo calculations helped determine the source profile and the source-detector coupling.

The measurements were made in October 1982, and the calibration and modeling done in 1983 resulted in an estimate of 2 to $7 \mathrm{~kg}$ of fuel in the demineralizer. This value is comparable with the HEDL results. These estimates are significantly below the criticality limit (about $80 \mathrm{~kg}$ ). Attempts have been made to obtain samples of the demineralizer material for analysis at Oak Ridge, and it will be very interesting to compare the destructive analysis with the nondestructive measurements.

The unique advantage of the Los Alamr . measurement nethod is the data collection in real time with small, battery-powered portable instruments. Now that the $\operatorname{Be}(\gamma, \mathrm{n})$ detector is calibrated, future data analysis should take only a few days.

We aiso have consulted with EG\&G on methods to measure the amount of fuel removed from the reactor core. We propose active and passive neutron measurements; however, the use of poisons for criticality control will complicate the nondestructive measurement of the removed materials. Further measurements await direction from EG\&G.

D. Nevada/Nevada Test Site (NTS) (P. E. Fehlau, C. E. Moss, D. C. Amsden, J. Martinez, and K. P. Dutcher, Q-2)

1. SNM Vehicle Portal Monitor. At the NTS, vehicles need to be monitored for the presence of SNM wherever nuclear devices are assembled, stored, and tested. Because access to SNM may be possible for extended time periods at the storage and assembly areas, installation of fixed SNM vehicle monitors can provide quick, uniform, and effective monitoring to enhance diversion safeguards. These monitors need only detect SNM subcomponents of test devices, a task that is relatively easy to accomplish.

At the request of DOE Nevada Operations Office (DOE/NV), we have developed a vehicle SNM portal monitor (Fig. 4) consisting of a gamma-ray detection system operated under microprocessor control to detect transient increases in gamma-ray intensity in the space betv'een the detector pillars. Intrusion sensors inform 


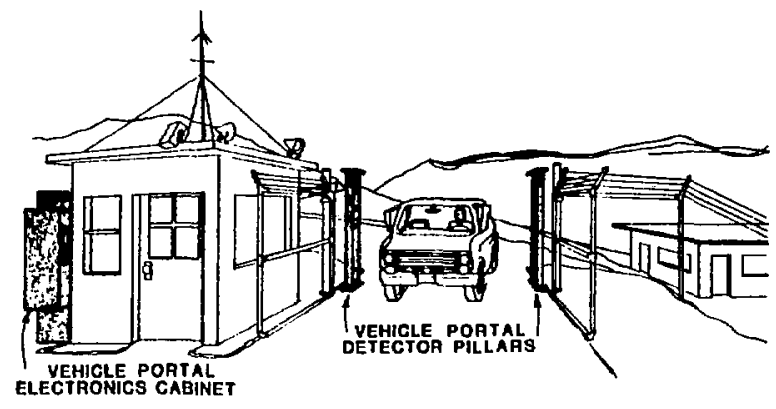

Fig. 4. The vehicle SNM portal monitnr comprises an array of gamma-ray detectors in pillars beside the path of exiting vehicles and an electronics cabinent containing the signal-processing and control electronics located near the guard station.

the controller when to monitor a vehicle. At other times, the monitor tracks the ambient gamma-radiation intensity. As a vehicle passes between the pillars, the system controller compares the detector response to the expected response derived from the most recent background intensity. Significant increases in response produce an alarm that indicates the presence of SNM. The details of the monitoring physics and detection procedures that apply to this monitor, and vchicle monitoring in general, are reviewed in Ref. 12.

Installation of the NTS monitor awaits completion of other new security measures at the NTS assembly area. In the meantime, a prototype was constructed and tested at Los Alamos, and a similar monitor developed for the Defense Nuclear Agency (DNA) has been installed and is operating at a US naval weapons storage facility. The DNA monitor (Fig. 5) is the original vehicle portal from which the NTS monitor was adapted. Both monitors are self-contained systems engineered to operate outdoors in a hot climate with intense solar illumination.

The DNA monitor has chilled water cooling for the detectors and a conventional sliding interval detection logic program. ${ }^{13}$ The NTS monitor incorporates thermoelectric detector cooling as well as a new transient signal detection method-the sequential probability ratio test (SPRT). ${ }^{3}$ The new techniques applied in the NTS monitor will be compared with the more conventional ones in the DNA monitor. The convenience of thermoelectric cooling is already obvious both in minimizing installation effort and in providing quiet operation.

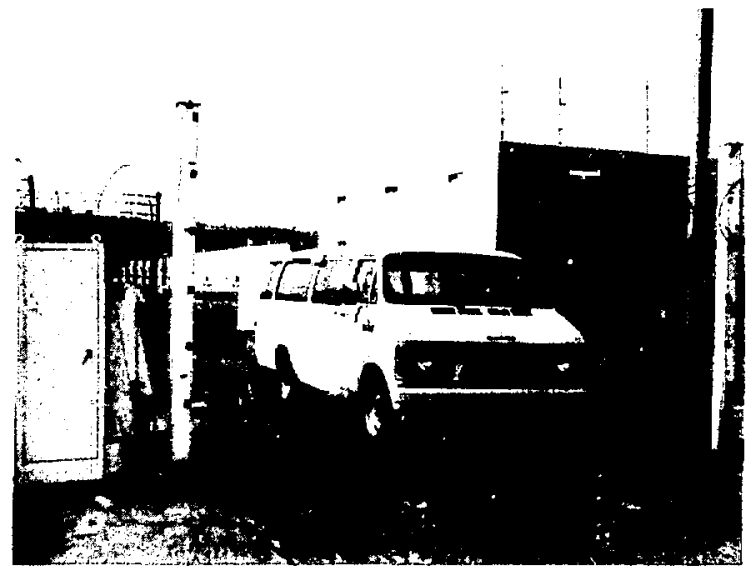

Fig. 5. A prototype vehicle SNM portal monitor for the DNA is operating at a Naval weapons storage facility. The monitor serves to detect inadvertent removal of nuclear weapons. Operational evaluation of this monitor is providing valuable information for further development of the NTS monitor.

2. SNM Monitoring at Ground Zero. The relatively short period of time that a test device resides at a ground-zero location before a shot does not justify installation of fixed monitors. These locations will be hand monitored with existing monitors. We are providing a hand-held-monitor user's manual to support training and supervision of hand-held monitor users.

\section{Device SNM Verification. The DOE/NV becomes} the final custodian of SNM in nuclear devices tested at the NTS. Because this office has no part in the construction and assembly of the devices, it must depend on others for quantitative verification of the amount of contained SNM. Independent assay of the test device SNM requires a technique that is reasonably accurate, rapid, and simple enough that data can be acquired without additional staff.

Our investigation of verification procedures applied during manufacture and assembly of a nuclear test device provided a bench mark goal for final device verification methods. We investigated several potential NDA methods that could be applied to assembled devices and estimated the verification accuracy in each case. A candidate technique worthy of further investigation is based on using an electron linear accelerator to interrogate each test device with neutrons or gamma 
rays. The interrogation produces a quantity of fission neutrons by neutron fission or photofission that is related to the amount of SNM. This technique can be rapid, accurate, and operated by personnel now stationed at the assembly area. The accelerator would not only provide verification data but would also be suitable for rapid radiographic analysis, thereby reducing the time now required for radiography with isotopic sources.

E. Oak Ridge/Y-12 Facility-Automated NDA Instruments for In-Line High-Enriched Uranium (HEU) Accounting (P. A. Russo, R. B. Strittmatter, E. L. Sandford, M. M. Stephens, J. K. Sprinkle, and R. W. Slice, Q-1)

1. Introduction. We are participating in a cooperative NDA instrumentation program at the enriched uranium recovery facility of the $\mathrm{Y}-12$ plant operated by the Union Carbide Corporation/Nuclear Division (UCC/ND) in Oak Ridge, Tennessee. This program, which began in 1981, has involved instrumentation to address three nuclear materials accounting needs identified by facility personnel.

- Measurement of the HEU inventory in the 1300 secondary solvent-extraction system

- Measurement of the HEU concentration in the secondary intermediate evaporator that precedes the 1300 solvent-extraction system

- In-line measurement of uranium concentration in solutions

Operational evaluation of instruments that address the first two specific needs has recently been completed, and an instrument that addresses the third, generic need has been installed. We designed all three instruments to measure uranium concentration in-line and to deduce HEU inventories automatically or provide process control information. ${ }^{14,15^{*}}$ Although function and design differ, the instruments share a common method of radiation detection, uranium concentration assay, and measurement control. Each instrument uses a computerautomated data acquisition system to obtain and analyze $\mathrm{NaI}(\mathrm{Tl})$ gamma-ray spectra characteristic of ${ }^{235} \mathrm{U}$, the primary radiation source in these solutions.

2. The EUREKA Instrument. The 1300 secondary solvent-extraction system inventory is measured by the EUREKA (Enriched URanium Extraction Kolumn Assay) instrument. EUREKA combines measured

\footnotetext{
*Reference 15 is available on microfiche from AIChE.
}

uranium concentrations in the solvent-extraction columns with a library of process design information to determine the HEU in entories in the external plumbing and in the columns. Concentration measurements are made during pulsed operation before shutdown for inventory and in the static mode after shutdown.

EUREKA comprises six shielded NaI(Tl) detectors mounted along the solution-bearing length $(9 \mathrm{~m})$ of any one of the three columns of the solvent-extraction system. The six detectors mount on brackets clamped to (or adjacent to) each column. The detectors are moved from column to column to perform the assays in count times of $600 \mathrm{~s}$. Column inventories are determined from the measured vertical concentration profile. The column inventory is the numerical integral of the product of column concentration and volume element. Figure 6 shows the EUREKA electronics installed in a remote, environmentally controlled area at $Y-12$. Figure 7 is a photograph taken in the $\mathrm{Y}-12$ solutiou recovery area showing a shielded detector mounted on a stainless steel section of a solvent extraction column. Details of the mechanical and electronic designs, the calibration of the concentration assay, the measurement control program for operation in the plant, and the operational evaluation procedures are described elsewhere. ${ }^{14-16}$

EUREKA was installed in December 1982 and evaluated throughout 1983. Evaluation of the inventory measurement involved carrying out the pulsed- and static-assay sequences and then dumping the solution components of the entire solvent-extraction system. The dumped components were analyzed externally to

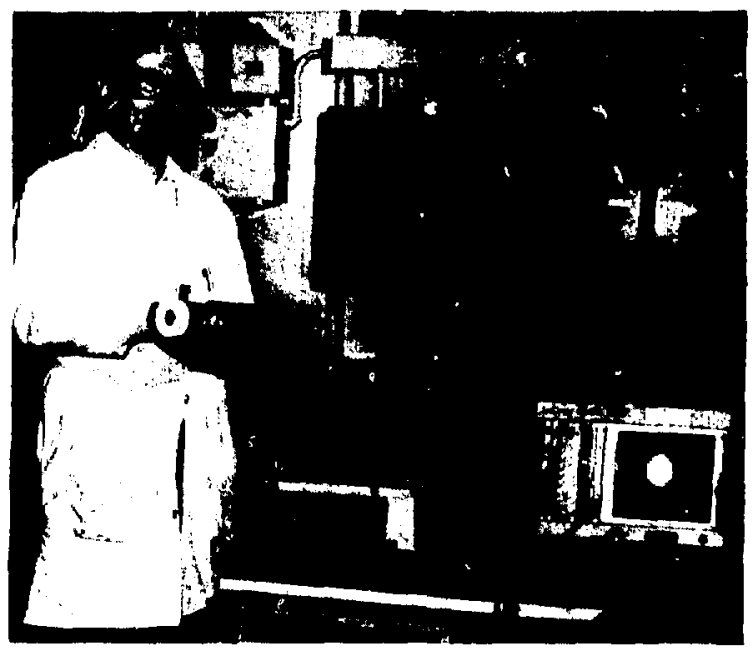

Fig. 6. EUREKA electronics installed at $Y-12$. A Y-12 employee holds one of the shielded EUREKA detectors. 


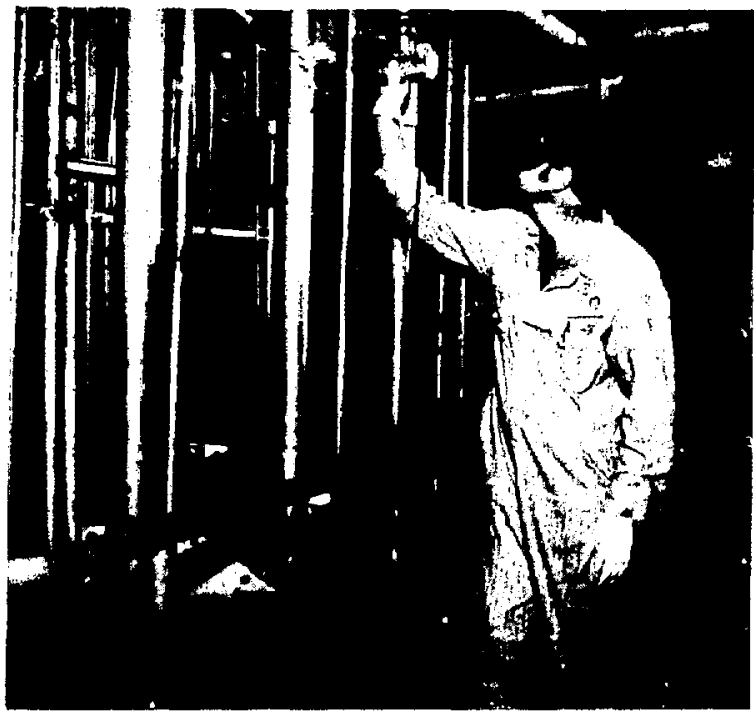

Fig. 7. Operator at the $Y-12$ HEU recovery facility shown positioning a EUREKA detector on the extraction column of the secondary solvent-extraction system.

determine solution volumes and uranium concentrations, to obtain reference values for comparison with the EUREKA inventory assays. The evaluation included five comparison dumps.

Comparison of the EUREKA inventory results with direct measurements indicates an overall average bias of $1.6 \%$ in the EUREKA column inventories (Table I). The largest individual average bias $(3.7 \%)$ was observed for the extraction column. The HEU inventories deduced by EUREKA for the plumbing external to the solvent extraction columns are larger than the dump results by $13 \%$. However, because the plumbing inventory is about
$20 \%$ of the total inventory, the additional bias introduced in the total EUREKA inventory is only about $2 \%$. Some of the bias must be attributed to partial draining of the plumbing lines (assumed to be full) that occurs following system shutdown.

Routine use of the EUREKA instrument with simplitied operating procedures started in January 1984.

3. Evaporator Monitor. An assay of uranium concentration in the secondary intermediate evaporator is desired to measure and minimize the HEU holdup caused by overconcentra is $\mathrm{n}$ and precipitation of solids. This monitoring also helps assure the desired uranium concentration for the solvent-extraction feed. We installed a shielded $\mathrm{NaI}(\mathrm{Tl})$ detector on the return loop of the recirculating evaporator and an automated instrumert to provide a near-real-time read-out of uranium concentration. The electronics cabinet and the shielded $\mathrm{NaI}(\mathrm{Tl})$ detector for the evaporator monitor are shown, installed at $Y-12$, in Figs. 8 and 9. The electronics equipment includes a digital read-out of uranium concentration that is used by the operator for evaporator control. Read-out updates occur, typically, at 30-s intervals, and more timely operator control of feed and product flow rates is possible with the information provided.

This instrument was installed in December 1982 and evaluated during 1983. Sampling difficulties related to the rapid circulation of hot solutions make a rigorous evaluation of assay performance difficult; however, in spite of these difficulties, the evaluation showed that the monitor performance was well within the required $10 \%$ accuracy. During several operating periods, samples were withdrawn from the return loop of the evaporator, and the results of the laboratory analysis of the uranium

\begin{tabular}{|c|c|c|c|c|c|c|}
\hline \multirow{2}{*}{$\begin{array}{l}\begin{array}{l}\text { Column Type } \\
\text { (No. Averaged) }\end{array} \\
\text { Extraction (5) }\end{array}$} & \multicolumn{2}{|c|}{$\begin{array}{c}\text { Column } \\
\text { Ave. Ratio } \sigma^{*}\end{array}$} & \multicolumn{2}{|c|}{$\begin{array}{c}\text { Plumbing } \\
\text { Ave. Ratio } \sigma^{*}\end{array}$} & \multicolumn{2}{|c|}{$\begin{array}{c}\text { Total } \\
\text { Ave. Ratio } \sigma^{a}\end{array}$} \\
\hline & 1.037 & 0.084 & 1.053 & 0.322 & 1.049 & 0.148 \\
\hline Strip (5) & 1.010 & 0.026 & 1.310 & 0.166 & 1.049 & 0.019 \\
\hline Backwash (5) & 1.060 & 0.031 & 1.018 & 0.360 & 1.000 & 0.046 \\
\hline All Columns (15) & 1.016 & 0.052 & 1.127 & 0.305 & 1.032 & 0.087 \\
\hline
\end{tabular}




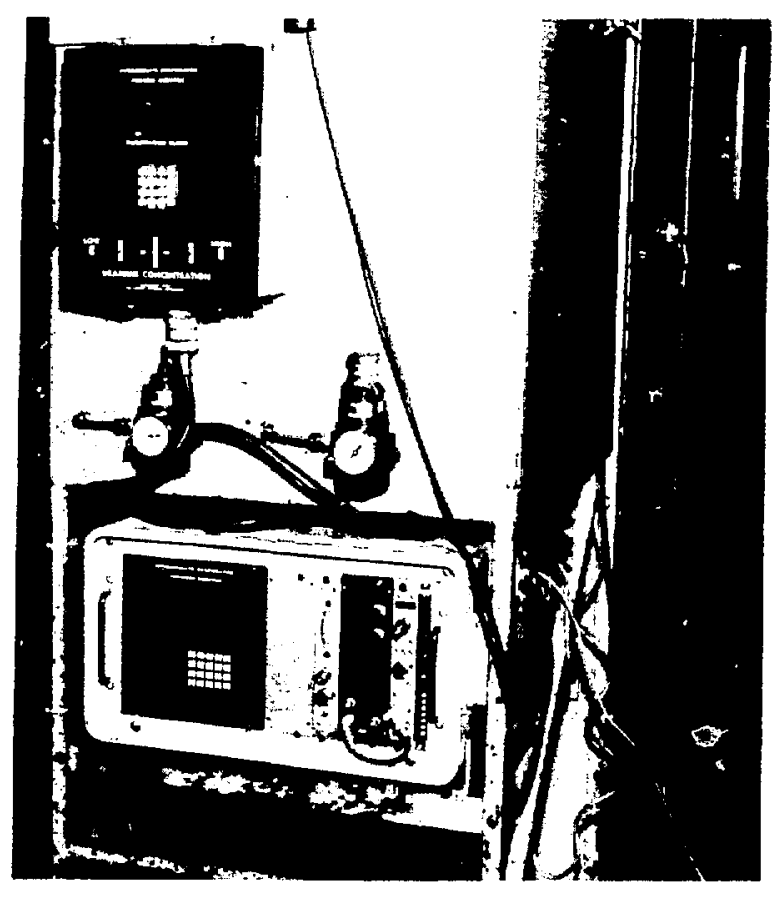

Fig. 8. Electronics for the uranium concentration monitor for the secondary intermediate evaporator installed at $\mathrm{Y}-12$. The LED concentration readout is at the upper left.

concentration of these samples by nondestructive highresolution gamma-ray' assay are shown in Fig. 10. These data indicate an average bias in the monitor assay of approximately $1 \%$ with a sample relative standard deviation (RSD) of $2 \%$.

This instrument has performed accurately and reliably since its installation and is in routine use by the operator. Because long-term performance criteria have been satisfied, automatic control of the evaporator operation can now be considered.

4. In-Line Monitors of Uranium Concentration. In September 1983, we installed two monitors to measure uranium concentration during pulsed operation of the 2300 secondary solvent-extraction system. The detectors, hardware, and electronic components for these monitors are the same as those used in the uranium concentration monitor for the $\mathrm{Y}-12$ secondary intermediate evaporator; ${ }^{17}$ however, in this application, the data acquisition system simultaneously acquires and analyzes two spectra. For the purpose of process monitoring, concentration results are provided in near-real time to two enlarged LED displays. The assay results also are transmitted to a PDP 11/23 computer, which is

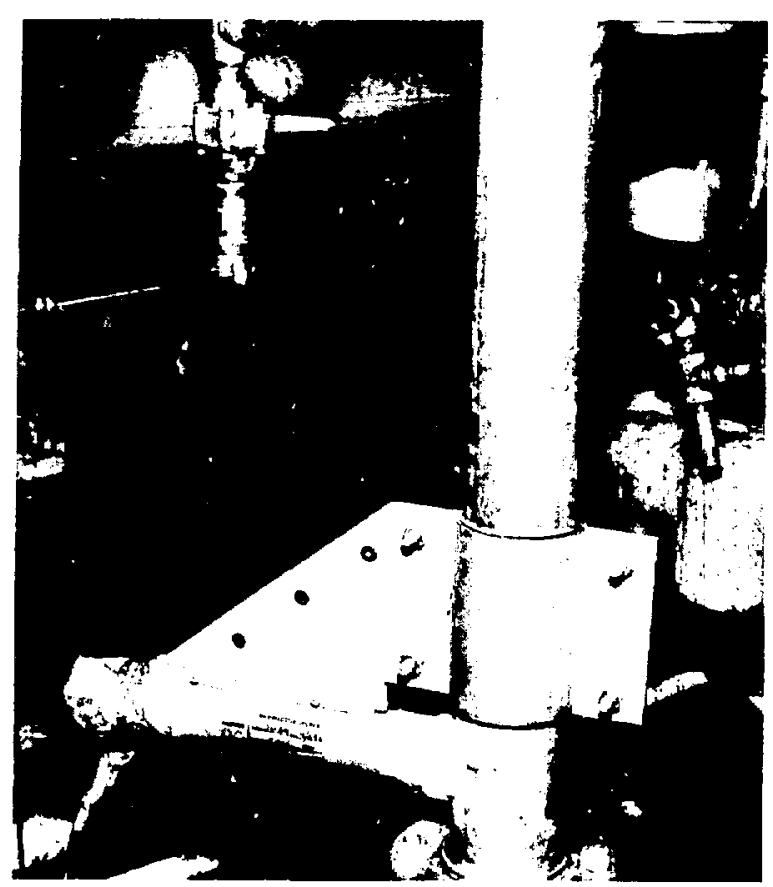

Fig. 9. The shielded NaI(TI) detector mounted on the ret*-a loop of the secondary intermediate evaporator.

programmed to automate control of the solvent-extraction system. This control capability is a demonstration effort carried out by the Y-12 Development Division, which also is comparing the monitor performance to the photometric uranium monitor currently being tested for process control applications under the Enriched Uranium Recovery Improvements Project.

The monitor calibrations were verified in December 1983 using a uranium reference solution in the standard cell built by Los Alamos. The two monitors are operating in the continuous assay mode (120 s/cycle) on the organic product of the extraction column and on the aqueous product of the solvent-extraction system. The latter monitor will be moved temporarily to an active location on the extraction column, where the concentration is substantially more sensitive to column operation parameters, and may prove to be more appropriate for process control. However, evaluation of the monitor performance will take place with the detectors in the original positions because sampling is less complicated for single-phase solutions. 


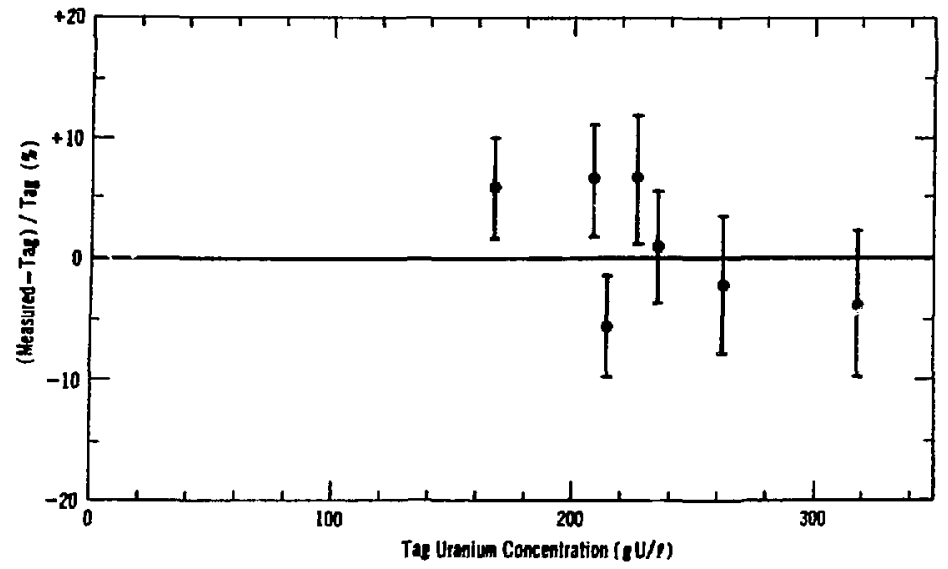

Fig. 10. Percentage deviation between 30-s evaporator monitor "measurement" and external sample assay ("tag") vs external sample assay. The error bars ( $1 \sigma)$ are calculated from counting statistics.

Evaluation of the monitors for the in-line assay of uranium concentration is being carried out by the Y-12 Nuclear Materials Control and Accounting Department to assess applications to possible (future) near-real-time accounting. During the 3-month period following monitor installation, samples were withdrawn from the aqueous product stream phase separator (approximately 1.5 min downstream from the monitor detector location) and assayed externally for comparison with the monitor results. The monitor and sample assays are shown for comparison in Fig. 11. The disírepancy between the monitor and sample assays on September 23
Fig. 11. Uranium concentration vs time for 100-s monitor assays of the 2300 system aqueous product (solid points) and external assays of samples withdrawn from the aqueous product stream (open points). The error bars in the monitor results represent the random uncertainty $( \pm l \sigma)$ computed from counting statistics. The error bars in the external assay give the estimated overall random uncertainty $( \pm l \sigma)$ in this result.

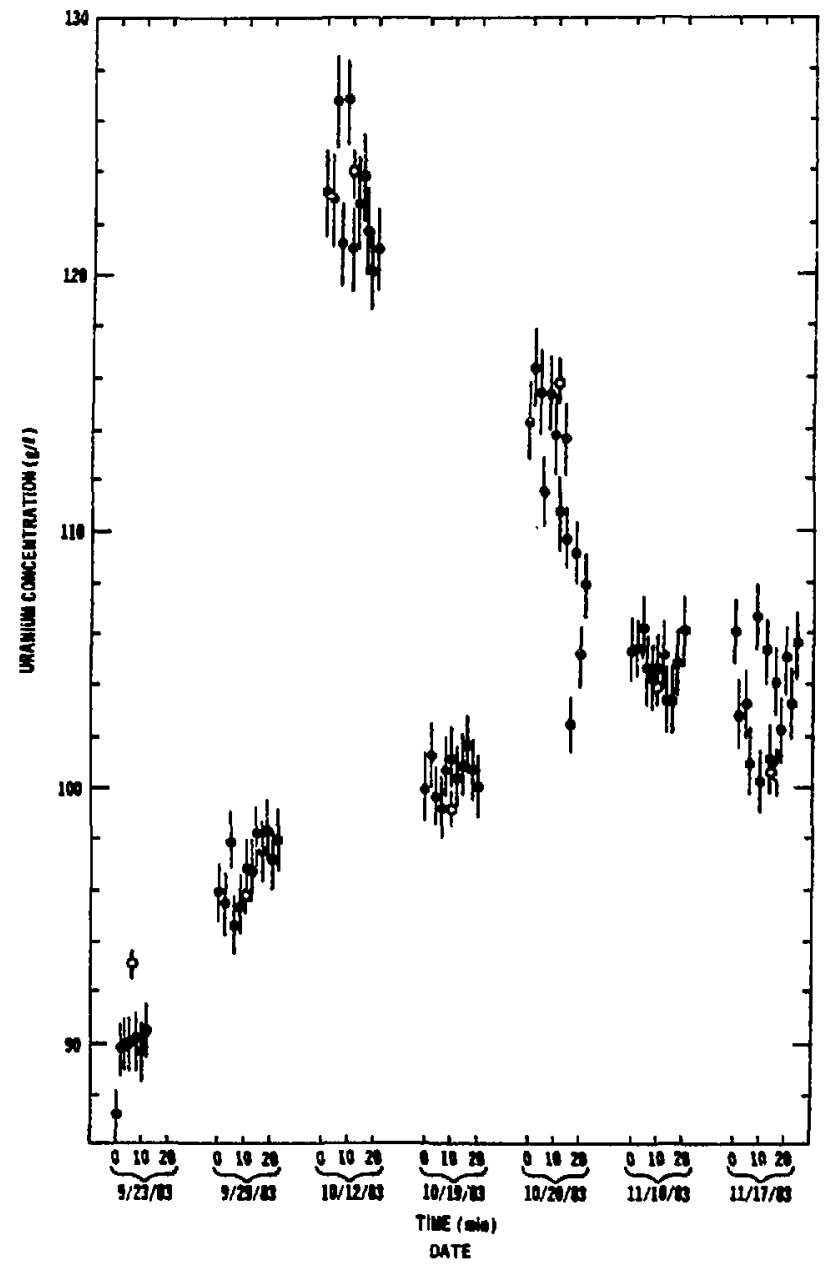


mav be due to the presence of saturated organic solution $(120 \mathrm{~g} \mathrm{U} / \mathrm{l})$ in the phase separator at the time of sample withdrawal. Evaluation of both monitors will continue during 1984.

\section{F. Oak Ridge/Consolidated Fuel Reprocessing Pro- gram (CFRP)}

1. Integrated Equipment Test (IET) (J. Barnes and C. Coulter, Q-4). We are assisting CFRP personnel in defining experiments to enhance MC\&A system performance. These tests will be part of the IET program at Oak Ridge, which will test and demonstrate, in a nonradioactive environment, new technologies applicable to fuels reprocessing. Proposed MC\&A technoiogies can be tested for extended periods using both state-of-the-art and advanced measurements and data processing systems.

\section{Breeder Reprocessing Experimental Test (BRET)} Program. The BRET Facility is planned as part of the Fuel Manufacturing and Examination Facility (FMEF) under construction by the Westinghouse Hanford Company near Richland, Washington (Fig. 12). When finished, these facilities (including the Fast Flux Test Facility, FFTF) will represent a complete breeder reactor fuel-cycle demonstration.

a. MC\&A Design (J. W. Barnes and C. A. Coulter, $\boldsymbol{Q}$-4). We are working with CFRP personnel to define optimum systems for MC\&A in a fast breeder fuel cycle. We have developed a conceptual design description for an MC\&A system (Fig. 13) that would be part of a 15 metric ton/yr fast breeder fuel-reprocessing facility. Performiance of this conceptual MC\&A system has been analyzed to determine its capabilities and define components that limit materials accounting sensitivity.

Los Alamos also is reviewing the MC\&A system design and operation for the BRET and is serving as a consultant to Westinghouse Hanford. We are examining the impact of equipment and process design on accounting sensitivity in support of plant design efforts. Materials transfer, sampling, and measurement alternatives are being evaluated.

b. Instrumentation (G. W. Eccleston, G. E. Bosler, and H. O. Menlove, Q-1). Los Alamos has been asked to provide consultation on instrumentation for the BRET facility to measure irradiated fast breeder reactor (FBR) fuel assemblies, leached hulls, and centrifuge bowls. Several assay systems are under consideration including passive neutron coincidence counting and active neutron systems using one of the following sources: a Triga reactor, a photoneutron source, a neutron generator, or a ${ }^{252} \mathrm{Cf}$ shuffler.

The hexagonal fue: assemblies contain between 5.1 and $8.1 \mathrm{~kg}$ of ${ }^{239} \mathrm{Pu}$ a. $\mathrm{nd}$ will have cooling times of I year or longer before measurement. The leached hulls are sheared pieces of stainless steel fuel cladding contained in a perforated metal basket; these are expected to contain $0.5 \mathrm{~g}$ of ${ }^{239} \mathrm{Pu}$ in $13 \ell$ of hulls. Assay precisions of

Fig. 12. Artist's rendering of the FMEF.

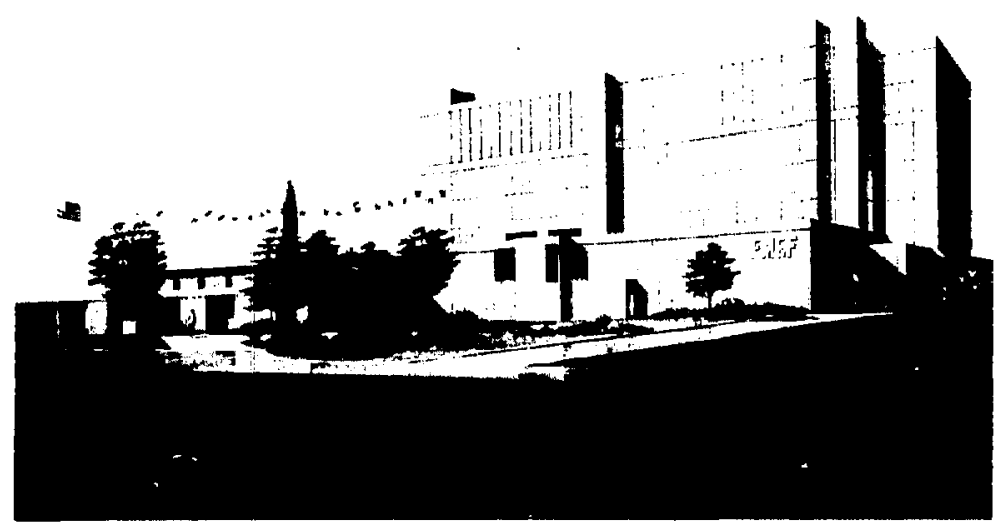




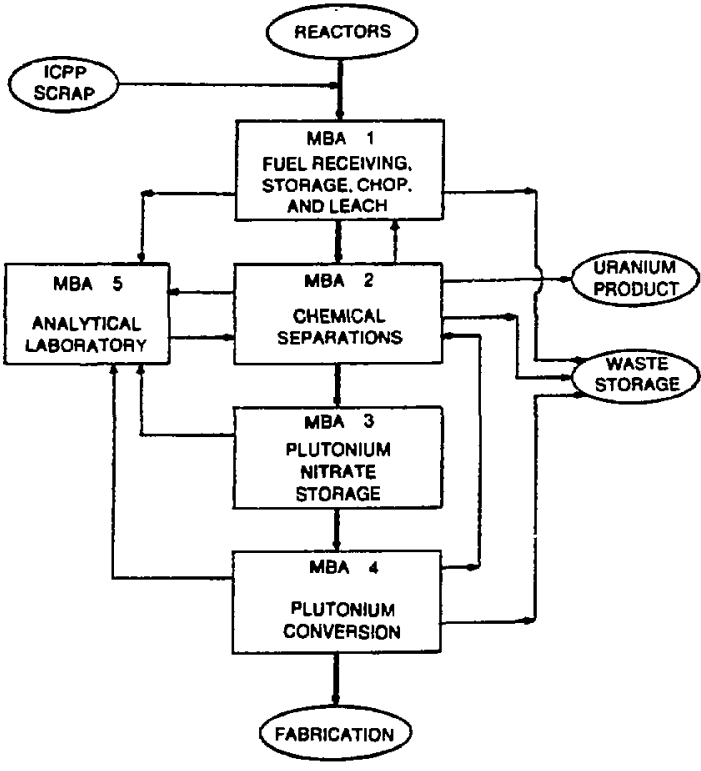

Fig. 13. Material balance areas.

$5 \%$ or better are required in measurement times between 0.5 and $2 \mathrm{~h}$.

We recommended a combination passive-active neutron neasurement system for assay of the fuel and leached-hull samples. This system would use passive neutron coincidence counting to measure plutonium in the leached hulls. The fuel-assembly measurements require an active neutron technique using high-energy neutrons for penetrability. The large plutonium loadings in these fuel assemblies will provide enough fissions from an active neutron source to give adequate sensitivity; however, the source must have sufficient intensity to override the neutron background in the fuel.

We participated in a meeting in May to discuss the various instrument options. Selection and development of an assay system requires further input from the facility designers and calculational and experimental design programs. We are continuing to consult with the BRET designers at Westinghouse to better define the future instrumentation needs of this unique and interesting facility.

\section{G. Oak Ridge/Portsmouth Gaseous Diffusion Plant (GDP)}

1. Cascade Holdup Measurements (R. H. Augustson, Q-1). Large amounts of uranium can be deposited in the operating equipment of a gaseous diffusion enrichment plant, especially in cases where leaks have occurred in the normally air-tight containment. We collaborated with the Goodyear Atomic Corporation in a series of gamma-ray and neutron measurements on cascade equipment located at the Portsmouth, Ohio, plant. The purpose of the measurements was to define techniques for localizing deposits and quantifying the amount of uranium held up in the equipment. We analyzed the data from these measurements and reported the results at the Institute for Nuclear Materials Management/American Nuclear Society (INMM/ANS) meeting held at Hilton Head Island, South Carolina. ${ }^{17}$ Goodyear has made additional neutron measurements that will be combined with the previous results. The ongoing effort includes improvements in the portable gamma-ray equipment and development of an overall strategy for minimizing the time and manpower needed to make holdup measurements.

2. Materials Accounting (R. Gutmacher and D. Stirpe, Q-4; R. Picard, S-1). With Brookhaven National Laboratory (BNL), we are evaluating the materials accounting system at the Portsmouth GDP. The primary objectives of this work are to quantify the magnitudes of certain measurement errors (necessary to formally assess the plant's monthly inventory differences) and to recommend improvements in current practices.

One area of major interest is the accounting for uranium in waste streams. Specific problems related to measurement of uranium in dilute solutions (discarded through the holding pond and drainage ditch), vent gas (the stack), contaminated oils (the oil plot), and trap material (alumina and sodium fluoride traps) have been identified and are being addressed. We also are continuing to develop error-propagation methods for the estimated losses of material.

H. Oak Ridge/Portsmouth Gas Centrifuge Enrichment Plant (GCEP) (J. T. Caldwell, H. F. Atwater, W. Bernard, S. W. France, R. D. Hastings, W. E. Kunz, and E. R. Shunk, Q-2)

We are developing an active-neutron interrogation system to verify and assay large clsssified-waste containers generated at the GCEP. Initial assay specifications for the Classified-Waste Assay Monitor (CWAM) included (1) assay sensitivity about $10 \mathrm{mg}^{235} \mathrm{U},(2)$ waste package dimensions-a cube 4.5 to $5.0 \mathrm{ft}$ on a side, (3) assay accuracy about $\pm 20 \%$ for the 4.5 - to 5.0 -ft-cube waste package, (4) system also capable of $\pm 10 \%{ }^{235} \mathrm{U}$ 
assay of 55-gal. drums of alumina trap waste, and (5) assay of ${ }^{238} U$ (accuracy not specified) in the 55-gal. alumina drums but not required in the $4.5-$ to $5.0-\mathrm{ft}$ cubes.

A quantitative evaluation of the available technology including all known passive gamma, passive neutron, and active-neutron techniques led us to conclude that the Los Alamos-developed differential dieaway (DDA) pulsed active-neutron technique is the appropriate choice for this large-package, high-sensitivity fissile assay problem. This basic technology was developed previously for the closely related problem of assay of fissile isotopes in $208-\ell$ drums and $1.2-$ by $1.2-$ by $2.1-\mathrm{m}$ packages of transuranic waste. ${ }^{18-20}$

We carried out experimental studies of the spatial assay response of ${ }^{235} U$ in a $208-\ell$ drum filled with alumina in an existing Los Alamos-designed and -constructed DDA assay system now in routine use at the Oak Ridge National Laboratory (ORNL). We obtained both vertical and radial responses by placing hollow aluminum tubes vertically in the drum at four different radial positions and performing standard DDA pulsedneutron assay measurements at the indicated locations with a 500-mg ${ }^{235} \mathrm{U}$ sample. Only two of the 24 measurements deviated more than $10 \%$ from the average, and both of these were in the mid-drum location at the two height extremes. Because these two measurements represent only about $1 \%$ of the total drum volume, the assay response was within $\pm 10 \%$ of a volume-weighted average for about $99 \%$ of the drum volume (alumina mass was about $200 \mathrm{~kg}$ ).

We also performed detailed assay measurements on two large wooden boxes filled with actual failed-rotor waste. (To avoid classification difficulties, certain specific details regarding these measurements are omitted.) We used an existing Los Alamos large DDA assay system, ${ }^{20}$ now in routine use at the Rocky Flats Plant for transuranic (TRU) waste assay, for these measurements.

In the DDA technique, the fissile mass is generally proportional to the ratio of the shielded detector response to the thermal-neutron-flux nonitor response. We measured this ratio for a standard ${ }^{235} U$ foil placed in several locations within the 1.2-m-cube box, which was filled with failed-rotor debris, and for three different positions of the neutron generator within the assay chamber. These measurements were analyzed to remove all background. The results are presented in Table II. The last measurement in the table was a 1.5 -m-cube box containing an unknown amount of ${ }^{235} U$ dispersed in failed-rotor debris.

Using the 1.2-m-cube box data as a calibration standard, we estimated the unknown ${ }^{235} \mathrm{U}$ mass in this
TABLE II. Ratio of Shielded Detector Response to Thermal Flux Monitor Response

\begin{tabular}{lccc}
\hline & \multicolumn{3}{c}{ Position } \\
\cline { 2 - 4 } Configuration & 1 & 2 & 3 \\
\hline
\end{tabular}

1.2-m-cube box, foil at box

$\begin{array}{lll}0.442 & 0.415 & 0.403\end{array}$ center, under $1 \mathrm{ft}$ of debris

1.2-m-cube box, foil in

$\begin{array}{lll}0.322 & 0.355 & 0.457\end{array}$ corner, half height

1.2-m-cube box, foil in $\begin{array}{lll}0.227 & 0.273 & 0.629\end{array}$ corner, top

1.2-m-cube box, foil in $\begin{array}{lll}0.200 & 0.242 & 0.338\end{array}$ corner, bottom

\begin{tabular}{llll} 
Average Calibration & 0.298 & 0.321 & 0.457 \\
1.5-m-cube box & 0.524 & 0.695 & 0.912 \\
\hline
\end{tabular}

container, and the assays performed with the three independent positions of the neutron generator agree reasonably well. These measurements show a more than satisfactory minimum assay sensitivity.

Based on these experimental studies and Monte Carlo calculations, we have developed a conceptual CWAM design for the GCEP based on the DDA technique and configured to provide quantitative ${ }^{235} U$ assays for the 1.2- to 1.5-m-cube boxes of failed-rotor debris and the 208- $\ell$ drums of contaminated alumina trap material (Fig. 14). The assay chamber, which comprises 12 modular side units for ease in construction and handling, approximates a circular cross section to optimize spatial flatness of the assay response. The relative positions of graphite, polyzthylene, and ${ }^{3} \mathrm{He}$ proportional counters are shown in Fig. 15, as well as the location of the movable neutron generator subsystem. In this conceptual design the 208- $\ell$ drums and the 1.2- to 1.5-m-cube boxes would be rotated during a measurement to further enhance geometric uniformity of response.

Final design is scheduled for mid FY 1984, and final construction is to be completed in mid FY 1985.

\section{Richland/Fuels and Materials Examination Facility (FMEF) (R. Gutmacher, E. Kern, J. Hafer, D. Martinez, M. Roybal, and S. Smith, Q-4; S. Hsue and T. Sampson, Q-1)}

We are assisting Westinghouse Hanford Company in the design of the safeguards system for the Secure Auto- 


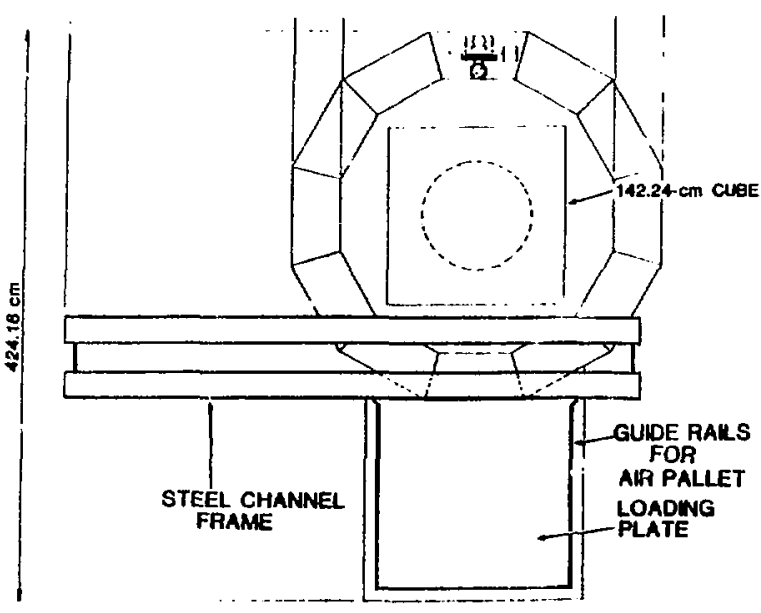

Fig. 14. Top view of the conceptual CWAM design, illustrating the relative sizes of the $1.2-$ to $1.5-\mathrm{m}$ large waste boxes and $208-\mathrm{R}$ drums within the assay system.

mated Fabrication (SAF) process line to be located in the FMEF at Richland, Washington. The SAF line will produce mixed uranium-plutonium oxide fuel beginning in 1987, with a design throughput of $6000 \mathrm{~kg} / \mathrm{yr}$. It is significant that, for the first time, integration of the process design and the safeguards system design have been achieved.

The safeguards system engineer must be knowledgeable of the process scenarios, operating schemes, and the process measurements and their associated errors when

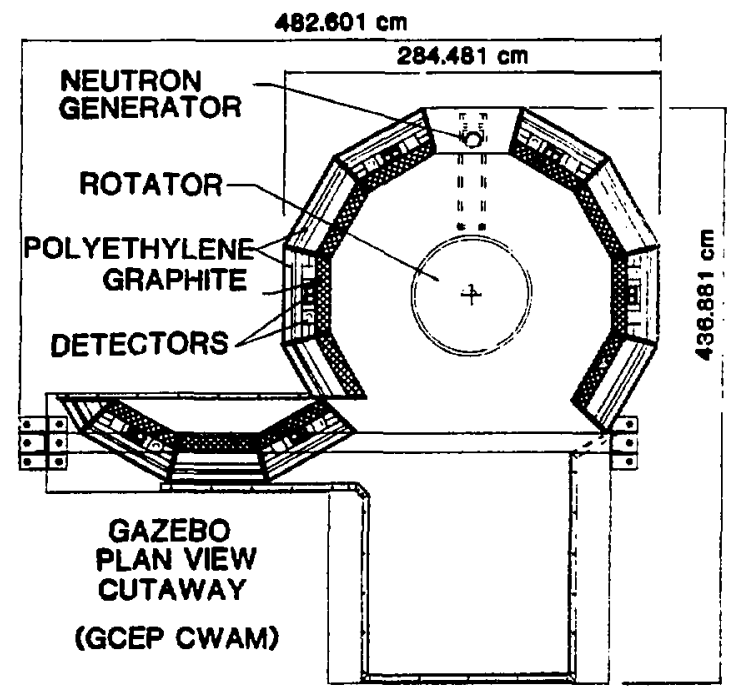

Fig. 15. Plan view of the conceptual CWAM design, illustrating the locations of all major system components. he disigns a safeguards system. It is desirable that the process operate smoohly, with minimum downtime and recycle streams, to simplify the materials control and accounting system. The process designer would like assurance that the process scenario and operating scheme will provide the required product output. In addition, the process designer would like to be aware of potential bottlenecks and know that adequate buffer storage capacity has been provided. We have used a computer-modeling and simulation approach that meets the needs of both the process and safeguards system designers.

We previously reported ${ }^{21}$ our results from modeling and simulation of two different process scenarios (Westinghouse's revisions 2 and 4 of the process flow diagrams); however, the process design has continued to evolve. We now have completed modeling revision 5 and are starting work on revision 6 of the powder and pellet operations.

We also completed a detailed study of the Boat Transport System, a vital link between several subsystems in the pellet operations part of the SAF line. A diagram of this system is shown in Fig. 16. The Debinding and Sintering Furnaces, Boat Unloading, Property Adjustment, and, of course, the Boat Transport System will operate $24 \mathrm{~h} /$ day, 7 days/wk. The Boat Inspection Station, which is manually operated, was scheduled to operate only 5 days/wk, one shift/day. We simulated three operating schemes for the Boat Inspection Station and found that a longer operating week and day will be required to provide the necessary supply of clean boats to Pressing and Boat Loading. We also established how full the two loop conveyors would be and how much of the time they would be in operation, compared with design criteria. We sent a draft report of our results to Westinghouse Hanford design engineers in August 1983. On the basis of our results, the engineers proposed revisions to the operating scheme in November. These revisions are now being studied at Los Alamos.

Until October 1983, our modeling effort concerned only the powder- and pellet-fabrication operations. At that time, sufficiently detailed and firm information on the fuel-pin operations became available, and we began work on a model. We expect to have initial results for Westinghouse design engineers early in 1984.

Process measurements data are being added to the powder-and-pellet operations model. We have assisted Westinghouse Hanford engineers in their selection of balances and load cells and in estimating the attainable measurement accuracy and precision. The combined process/measurements model will serve as the basis for design of the safeguards system in 1984. We will test various materials control and accounting schemes to 


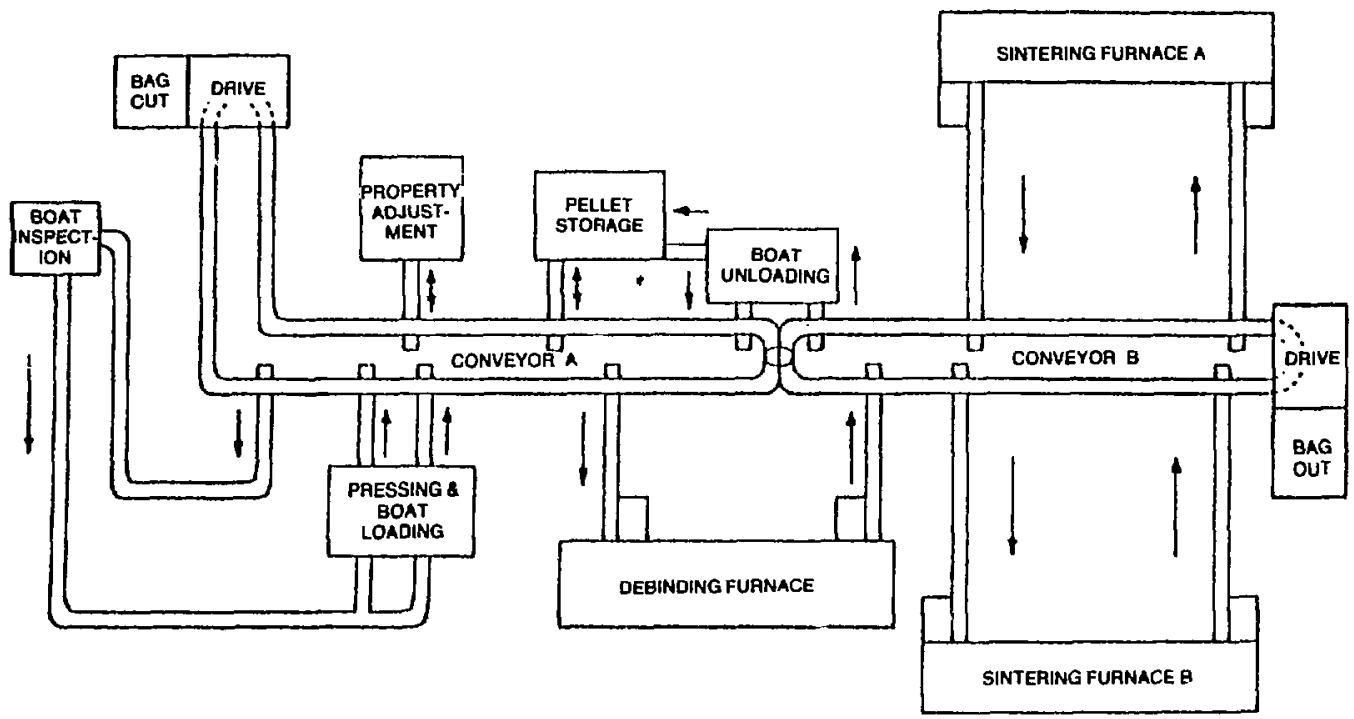

NOTE: ARROWS SHOW NORMAL

DIRECTION OF TAAVEL

ON INTERFACE CONVEYORS

Fig. 16. Boat transport system, Secure sutnnated Fabrication (SAF) line.

determine their sensitivity for materials loss detection. Because more than $2000 \mathrm{~kg}$ of plutonium oxide will be fabricated into fuel pellets annually, an effective safeguards system is of prime importance.

We have continued to discuss the need for NDA instrumentation for the SAF process with Westinghouse personnel. They supplied us with seven cans of mixedoxide powder that span a wide range of plutonium/uranium ratios. We analyzed the powders by high-resolution gamma-ray spectroscopy and submitted tniee samples from each can for detailed chemical and isotopic analysis. The results will show how accurately the plutonium/uranium ratio of blended mixed-oxide powder can be determined by NDA. If the results are favorable, we propose to construct and test a prototype instrument for use at the blender in the SAF line.

\section{J. Richland/Hanford PUREX: Screw Calciner Holdup Monitor (T. W. Crane, Q-1)}

The PUREX reprocessing plant operated by the Rockwell Hanford Company near Richland, Washington, will soon restart operation to extract plutonium from fuel irradiated in the Hanford production reactors. At the end of the extraction process, plutonium oxalate is dried to form $\mathrm{PuO}_{2}$ in a series of two continuous-feed rotary calciners.

To maintain materials accountability, it is necessary to measure the plutonium held up within the calciners. After considering both passive gamma-ray and neutron measurement techniques, we chose the latter as the most appropriate approach. While the primary goal of this instrument is the measurement of plutonium holdup at the end of a reprocessing campaign, daily process monitoring is possible and can provide useful information for process monitoring.

The passive-neutron technique selected uses detector banks placed on the outside of the glove box for a coincidence measurement. Three detector banks, extending beyond the length of the calciners, provide a uniform detection response. Two of the banks are shown in Fig. 17 before shipment to Hanford. Because of the variable and unknown moisture content of the plutonium oxalate, we designed a moisture measurement capability in to the instrument. As a process-monitoring feature, the instrument measures both plutonium holdup and moisture along the length of the calciner. The electronics associated with the detector include an HP-85 computer for data analysis.

The coriceptual design of the instrument was completed in 1982. The physical design, fabrication, and 


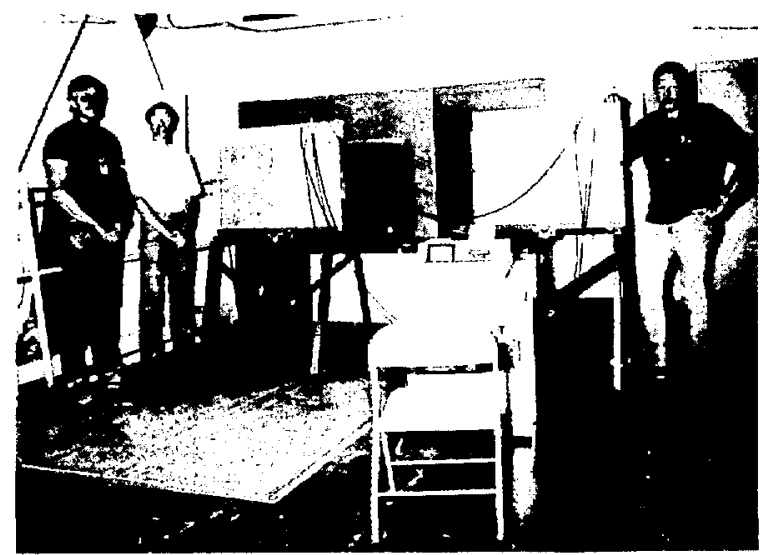

Fig. 17. Two of the neutron detector banks and associated electronics shown before shipment to PUREX.

testing were completed by the summer of 1983 . The instrument is now installed in the PUREX plant, and the initial calibration has been completed. Further calibration and evaluation will occur as PUREX proceeds to full operation during 1984.

K. Savannah River/Naval Fuel Materials Facility (FMF) (N. Ensslin, L. R. Cowder, G. E. Bosler, T. W. Crane, T. K. Li, and J. L. Parker, Q-1)

Los Alamos is providing a series of NDA instruments to the new. Naval FMF under construction by duPont at the Savannah River Plant (SRP). These instruments, which measure HEU in solids and solutions, will provide accountability, verification, and process-monitoring information at key measurement points through- out the facility. The variety of material categories expected in the facility and the techniques required to measure them are listed in Table III. To ensure nuclear material accountability, the instruments must provide in-line or at-line measurements of the highest possible accuracy. To ensure reliability, measurement techniques that have been proven in other facilities will be used. To meet these requirements, new versions of existing active and passive NDA instruments are being designed and fabricated. There is a paraliel program of optimization studies and laboratory measurements to assist the instrument design.

During 1983 we completed designs for all of the instruments listed in Table III except the Receipts Assay system and the Low-Density Scrap Assay system. Fabrication of the first shuffler for high-density scrap was begun and is now nearing completion. Fabrication of the first solution assay system and the waste assay systems will begin early in 1984. Procurement of computers, multichannel analyzers, high-resolution germanium detectors, ${ }^{3} \mathrm{He}$ gas-filled neutron detectors, and other major components is under way.

The adaptation of existing measurement techniques to the needs of the new facility has required improvements in hardware and software design. Two of the shufflers will fit under glove boxes and assay samples in the process line, requiring extensive modification of the assay chamber configuration, the californium source holder, and the stepping-motor controller. The shufflers and the gamma-ray assay instruments will use the same modular software routines for communication with the operator, the accountability computer, and peripheral equipment. This commonality will minimize software production and maintenance requirements at both Los Alamos and Savannah River.

An example of one of the new instrument designs is shown in Fig. 18, the High-Density Waste Shuffler that

\begin{tabular}{|c|c|}
\hline Material Category & Measurement Technique \\
\hline $\begin{array}{l}\text { Receipts material } \\
\text { High-level solutions } \\
\text { Low-level solutions } \\
\text { High-density scrap } \\
\text { Low-density scrap } \\
\text { Finished product } \\
\text { High-density waste } \\
\text { Low-density waste }\end{array}$ & $\begin{array}{l}\text { Passive neutron counting } \\
\text { High-resolution gamma counting } \\
\text { High-resolution gamma counting } \\
\text { Active neutron interrogation (shuffler) } \\
\text { Segmented gamma scanning } \\
\text { Active neutron interrogation (shuffler) } \\
\text { Active neutron interrogation (shuffler) } \\
\text { Far-field gamma counting }\end{array}$ \\
\hline
\end{tabular}




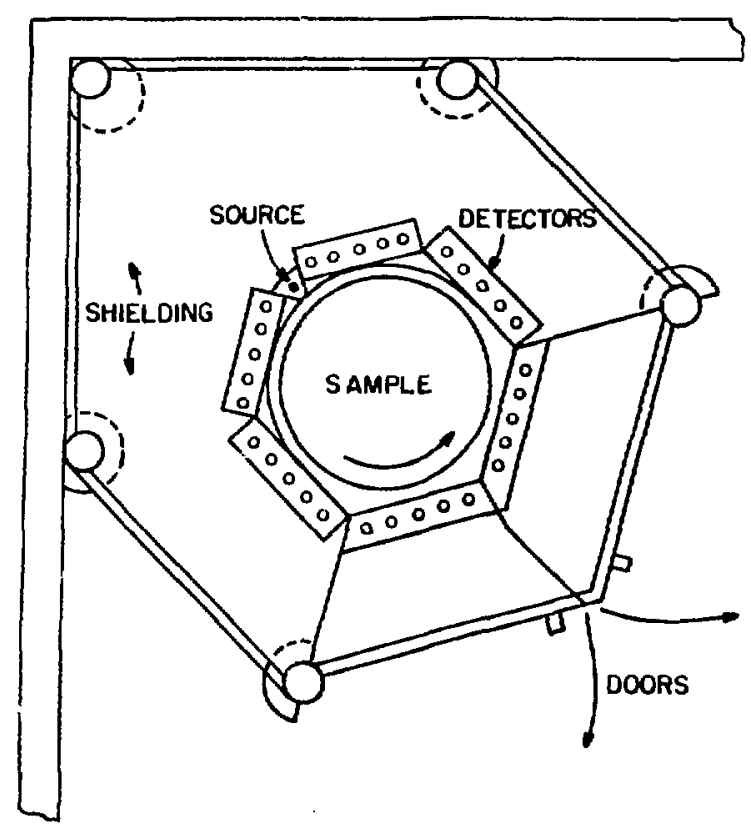

Fig. 18. A top view of the High-Density Waste Shuffler designed for use at the Savannah River Plant.

uses a californium neutron source to interrogate waste containers. Six banks of ${ }^{3} \mathrm{He}$ neutron detectors are located around the side of the waste container. Detector banks above and below the container help provide a uniform detection response. The californium source will move along the side of the container as it retates. The purpose of this instrument is to ensure that significant quantities of uranium are not discarded accidentally with the high-density waste.

Over the past year, supporting work for these new instruments has included the following studies:

- An error analysis for the in-line scrap and product shufflers

- Monte Carlo modeling of the shufflers to determine the required californium source strength

- An error analysis of the High-Level Solution Assay System to determine the required sample vial dimensional tolerances and required counting times 22

- Monte Carlo calculations of the shielding required by the shufflers to maintain acceptable personnel radiation doses

- Deadtime and pileup measurements with germanium detectors at high counting rates

- Design and characterization of NDA standards for the instruments
During 1984 work will continue on precision and stability tests, the use of high count rates, and standards fabrication. We will begin laboratory measurements to establish calibration curves and matrix correction factors. Acceptance testing, shipping, and installation are scheduled to start in the spring of 1985.

L. Savannah River/New Special Recovery (NSR) Facility (M. P. Baker, S.-T. Hsue, L. Speir, T. E. Sampson, S. Johnson, and T. Van Lyssel, Q-1)

The duPont Company is constructing a facility for the DOE/SRP to house the New Special Recovery process. This facility will recycle and recover off-specification plutonium, excess process waste, and process scrap and will be located on the fifth and sixth levels of an existing building whose lower levels contain a plutonium-reprocessing canyon. The layout of the NSR is shown in Fig. 19.

Input materials will be brought to the Feed Assay Room where total plutonium will be measured with NDA instruments. Materials will then be transferred into the glove box train for preparation before their dissolution in one of two dissolver/anion-exchange systems. Following dissolution, solutions are transferred directly to first-cycle canyon tanks or purified in anionexchange columns and then transferred to second-cycle canyon tanks. Required NDA measurements of dissolver/anion-exchange cabinet materials will be done in the Sample Assay Room with samples obtained from process vessels. Solid waste from the process will be transferred to the waste-handling cabinet and assayed for plutonium content with NDA. Liquid waste will be sampled, assayed for plutonium in the Sample Assay Room, and transferred to canyon tanks.

We have been working with duPont personnel since October 1982 to define an integrated system of modern, automated NDA instrumentation that will provide accountability and process-monitoring information to the NSR facility operators. This instrumentation will be developed and fabricated by several vendors including Los Alamos, Lawrence Livermore National Laboratory (LLNL), Savannah River Laboratory (SRL), and Mound Laboratory. Los Alamos will serve as system coordinator, combining the individual instruments into an integrated package and testing the system at Los Alamos for a 6-month period before its installation at Savannah River.

A major project during FY 1983 was the completion of a conceptual design study ${ }^{23}$ for the integrated system. The proposed system is shown in Fig. 20. The study 


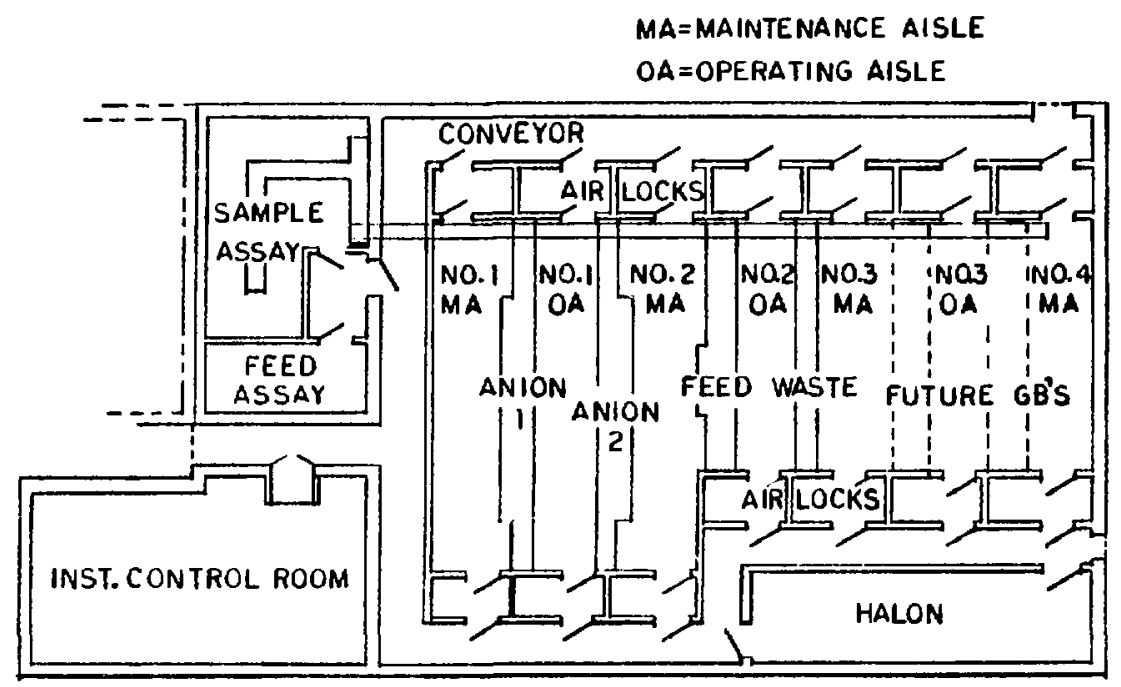

Fig. 19. Physical layout of New Special Recovery facility.

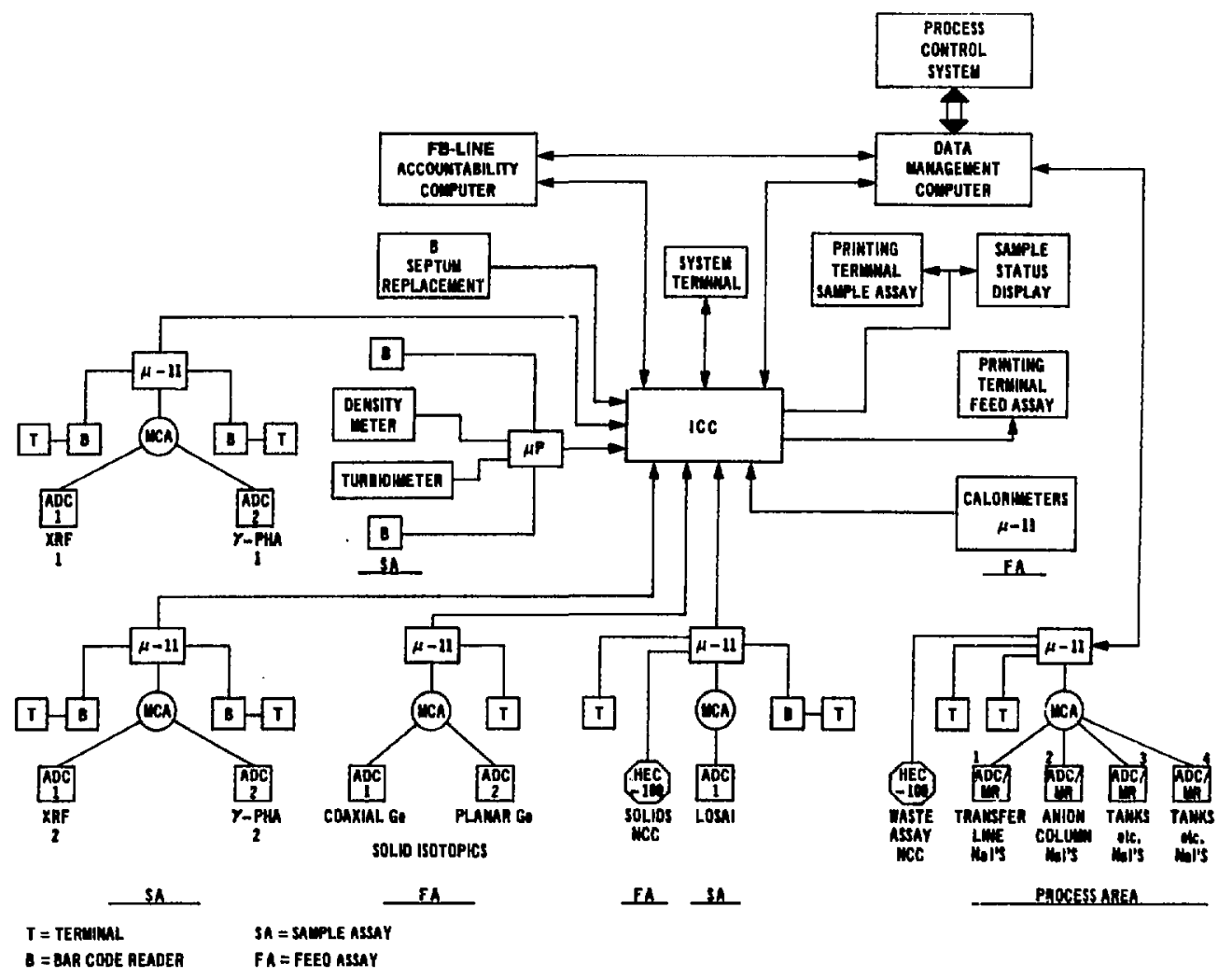

Fig. 20. Block diagram of the proposed integrated accountability system. 
proposes that the NDA instruments be microcomputerbased and report their assay results to a central instrument-control computer (ICC). The ICC is to be integrated into the facility's acccuntability and processcontrol computer network. These general concepts have been accepted by all parties, and the project planning is proceeding on this basis. Los Alamos is responsible for the software development of the ICC, the development of four major NDA instruments, and the system integration.

The integrated system is to be delivered to the SRP in September 1985. Therefore, whereas FY 1983 was devoted to planning and design, FY 1984 will be dedicated to instrument fabrication and software development. FY 1985 will emphasize the testing and integration of the system. Installation is scheduled for early FY 1986. An extensive performance evaluation is planned and will begin when the facility process and the integrated NDA systems have achieved routine operation.

This plan for implementing a near-real-time accounting system in a process environment represents a major step in the realization of the full capabilities of modern NDA instrumentation. As currently conceived, there will be no routine analytical chemistry for plutonium accountability at the NSR. We anticipate that the successful operation of this multi-Laboratory, integrated NDA system will point the way to future innovations in the design of near-real-time accountability systems.

\section{Savannah River/FB Line (J. W. Barnes, Q-4)}

We have developed a process simulation and materials accounting model of the SRP FB line that is used to evaluate alternative materials handling, measurements, and accounting methods. Plutonium nitrate solutions from the Savannah River fuel reprocessing operations are purified, concentrated, and converted tn plutonium metal in this facility.

We used the MODEL code, in conjunction with the GASP IV simulation language for event scheduling, to model the dynamic operation of the FB line on a VAX $11 / 780$ computer. The changes in materials-in-process for a single process unit, as simulated by the computer, are illustrated in Fig. 21. Sixty unit processes, incorporating approximately 80 equipment pieces and several hundred individual process steps, are included in the simulation. Figure 22 shows the complexity of a section of the FB line. Both batch and semicontinuous operations are simulated along with the dynamic variations that occur during plant operation. One month's

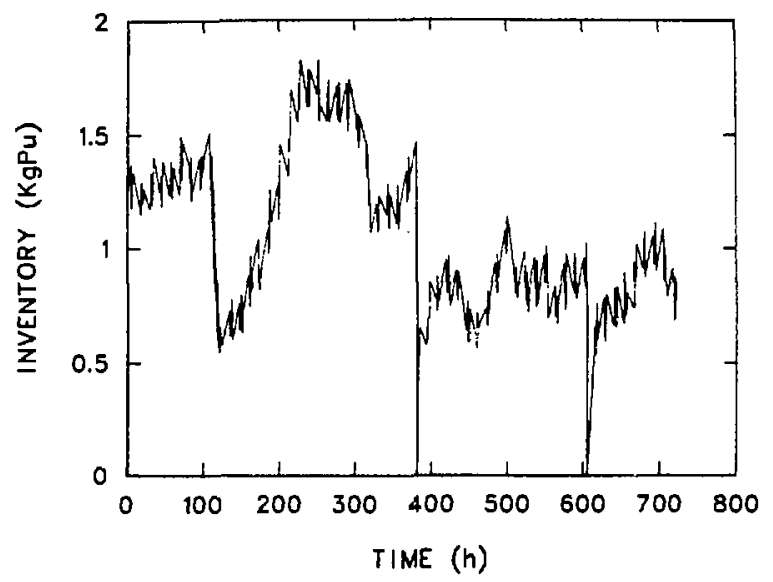

Fig. 21. Typical simulation results showing the variability of the plutonium inventory in the $\mathrm{M} 3$ precipitator.

operation of the complete process line can be simulated with approximately 30 minutes of computer time.

Process and measurements-error data were developed by Los Alamos and SRP personnel for use with the FB line model to compute materials balances and their associated variances. We assessed the capability of the present materials accounting system and proposed improvements in measurement methods, accounting procedures, and process operation that will be evaluated to determine their net effect on reducing errors in inventory estimates. Useful information obtained from this work includes

- Determination of inventory accuracy and associated errors and variances

- Estimation of the effect on materials accounting of unmeasured inventory accumulation (spills) within the process line

- Identification of primary error sources (in materials measurements) and their impact on materials accounting

- Evaluation of alternative process operating configurations, new measurement systems, or accounting methods for their effect on materials accounting accuracy

We have worked with Savannah River to identify operating changes or system modifications that can lead to improvements in materials control and accounting. This effort has proven highly valuable in illustrating how safeguards effectiveness can be improved, identifying key materials accounting points, and providing input for long-range project planning. 


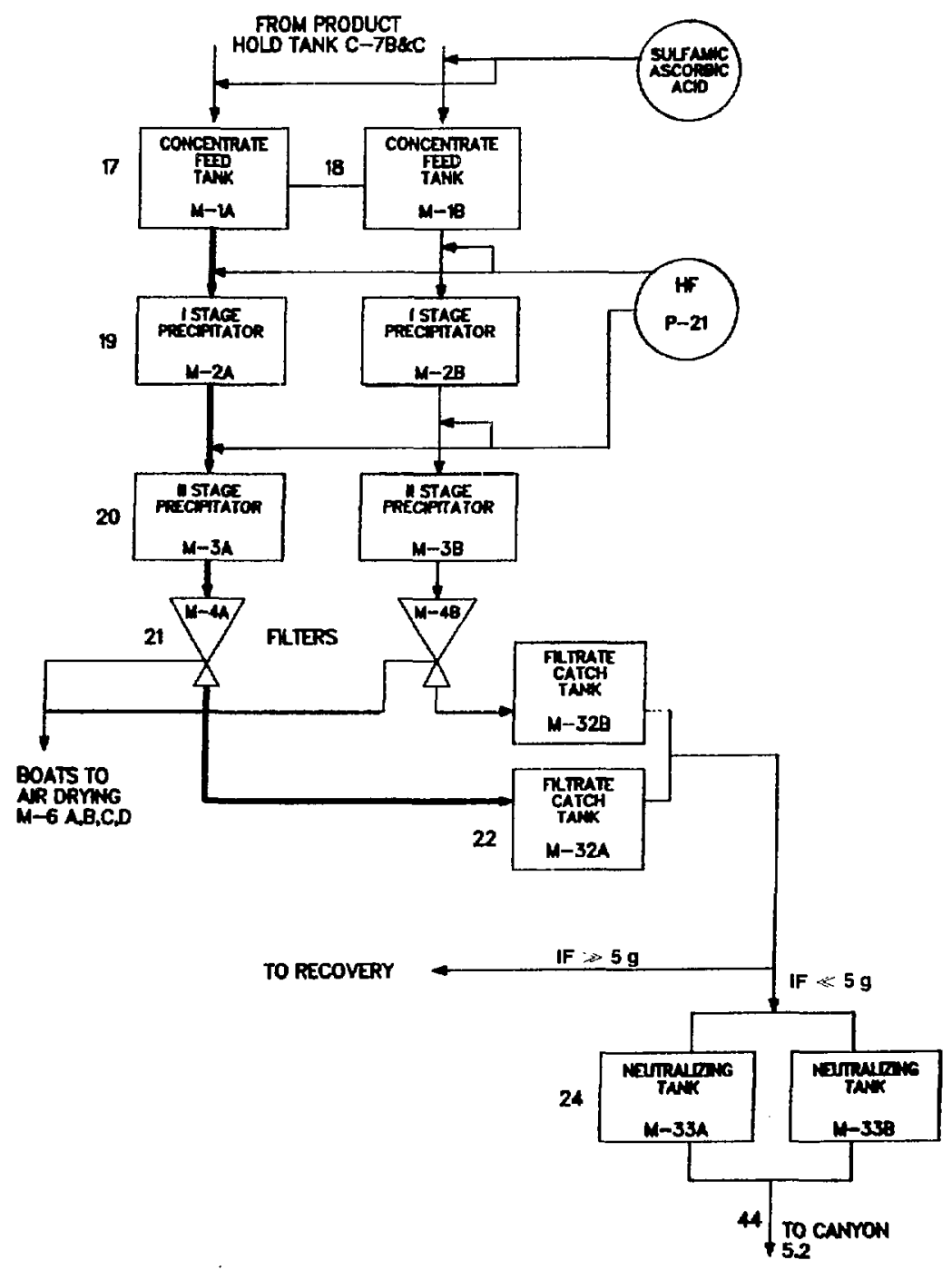

Fig. 22. Typical process flow sheet-plutonium precipitation.

N. Ad Hoc Field Assistance/Savanrah River Laboratory (SRL) (H. O. Menlove and J. K. Halbig, Q-l)

At the request of SRL and DOE/Savannah River, we assisted SRL in developing a portable assay system (Fig. 23) to improve the timeliness of the accountability of SNM in the SRL laboratories, to reduce the possibility for error in handling and disposing of laboratory SNM samples, and to provide measurement capability for miscellaneous types of small samples found in the laboratories. This system, the first to integrate neutron and gamma-ray measurements to give the conversion from
${ }^{240} \mathrm{Pu}$-effective to total plutonium, comprises several commercially available components mounted on a modified laboratory cart. The components are listed below.

- HEC-100 electronics (IRT Corporation)

- Inventory Sample Coincidence Counter (JOMAR, Inc.)

- Davidson Model 2056-4K MCA (D. Davidson Company)

- germanium detector (14\% efficiency) (Ortec)

- HP-85 minicomputer (Hewlett-Packard) for system control 


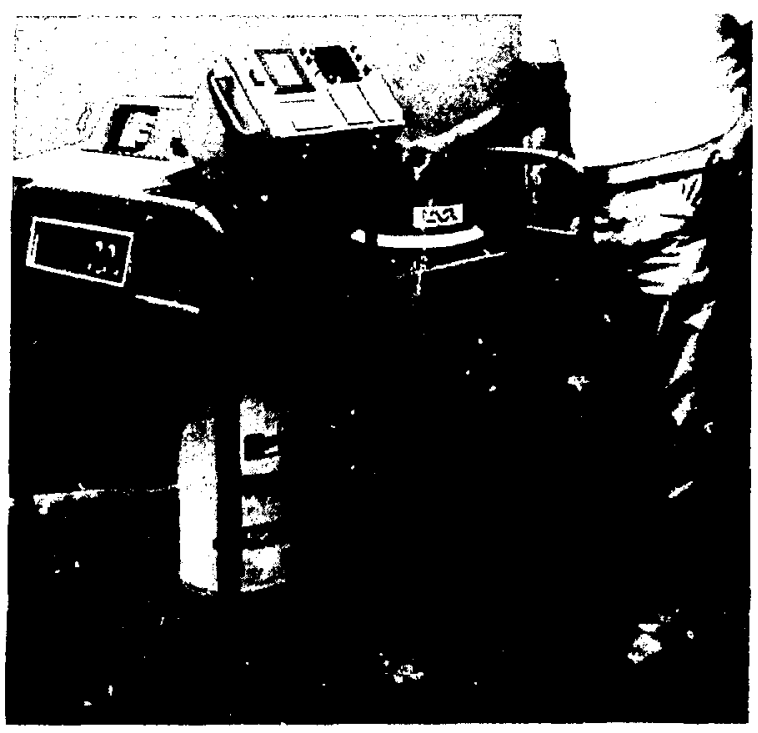

Fig. 23. SRL's Portable Assay System. The neutron coincidence counter is located at the rear of the cart on the lower shelf. The germanium detector for gamma-ray measurements is located beneath the neutron counter so the sample is positioned only once for both measurements.

SRL procured the components of the system directly from the individual vendors; Los Alamos assistance included

(1) demonstrating portable equipment developed for similar applications by the International Atomic Energy Agency (IAEA),

(2) developing specifications for the commercial procurement of the required NDA equipment,

(3) developing software in the HP-85 for system control and data analysis,

(4) providing training for SRL staff in the use of the NDA equipnent,

(5) assisting SRL staff in the setup and calibration of the equipment at SRL, and

(6) assisting SRL in the application of the system to measuring different types of SNM.

In application, the portable assay system is wheeled to the various chemical laboratories where plutonium is being used. The inventory is measured and compared with the plant tag values for the individual containers. Materials categories in the calibration include $\mathrm{PuO}_{2}$, plutonium metal, plutonium nitrate, $\mathrm{PuO}_{2}-\mathrm{PuF}_{4}$, and ${ }^{238} \mathrm{Pu}$.

Before final implementation, SRL sent a ${ }^{252} \mathrm{Cf}$ neutron source to Los Alamos for calibration and for cross referencing with the Los Alamos plutonium standards.
After this work was completed, the source $\left({ }^{252} \mathrm{Cf}, 4.18 \times\right.$ $10^{4} \mathrm{n} / \mathrm{s}$ on October 19,1983 ) was returned to SRL for use as a calibration normalization source.

Future assistance to SRL will include applications to additional materials types, improved calibration parameters, and upgraded software. In particular SRL has asked for assistance in applying the s/stem to determine the enrichment of HEU samples.

\section{SAFEGUARDS TECHNOLOGY TRAINING (C. Hatcher, Q-1)}

As part of the DOE Safeguards Technology Training Program, three training courses were presented at Los Alamos in 1983.

- Fundamentals of Nondestructive Assay of Nuclear Material

- Neutron Assay of Nuclear Material

- Gamma-Ray Assay of Nuclear Material

Each course was scheduled for 4-1/2 days and involved intensive laboratory exercises with a variety of NDA instruments to measure typical uranium and plutonium samples. Table IV shows the training course attendance for 1983. Seventy per cent of the students attending the 1983 courses were from DOE and its contractor organizations, up from $50 \%$ in 1980 . This increase is attributed largely to the fact that improved materials accounting (based on NDA techniques) has reached a point at DOE facilities where more trained people are required. Each training course presented in 1983 has been given at least once in previous years, but the courses are updated each year to include the latest in measurement techniques and commercially available instruments.

The course in fundamentals provides an introduction to the NDA of nuclear materials for both gamma-ray and neutron measurement techniques. Although designed primarily for professional level scientists and engineers with little or no background in NDA, the course also has proved useful to materials accounting supervisors, Nuclear Regulatory Commission (NRC) inspectors, and NDA technicians. Topics include gamma-ray and neutron interactions, uranium enrichment measurement, transmission-corrected gamma-ray assay, neutron singles counting, and neutron coincidence counting.

Both the neutron assay and the gamma-ray assay courses are intended for scientists, engineers, and technicians who wish to become familiar with advanced NDA techniques typically used in in-plant instruments. The neutron course covers neutron interactions and 


\begin{tabular}{|c|c|c|c|}
\hline \multicolumn{4}{|c|}{$\begin{array}{l}\text { TABLE IV. } 1983 \text { DOE Safeguards Technology Training Program } \\
\text { Attendees }\end{array}$} \\
\hline & $\begin{array}{c}\text { Fundamentals } \\
\text { of NDA }\end{array}$ & $\begin{array}{c}\text { Neutron } \\
\text { NDA } \\
\end{array}$ & $\begin{array}{c}\text { Gamma-Ray } \\
\text { NDA }\end{array}$ \\
\hline Argonne & - & $\mathbf{1}$ & - \\
\hline Battelle & 1 & - & 1 \\
\hline DOE & 2 & 1 & 2 \\
\hline duPont & $\mathbf{1}$ & 7 & 2 \\
\hline EG\&G & 1 & - & 2 \\
\hline Exxon, Idaho & 1 & 1 & - \\
\hline Goodyear & - & 1 & $\mathbf{1}$ \\
\hline IAEA & - & 2 & 4 \\
\hline K-25 & 2 & - & - \\
\hline Los Alamos & 5 & 4 & 3 \\
\hline LLNL & 1 & - & - \\
\hline NRC & 7 & - & 2 \\
\hline NFS, Erwin & 3 & 2 & 1 \\
\hline Other & - & 2 & 1 \\
\hline Rockwell, Hanford & 3 & 2 & 1 \\
\hline Rockwell, Rocky Flats & 2 & 1 & 2 \\
\hline Westinghouse, Hanford & 2 & - & 1 \\
\hline Y-12 & 1 & - & - \\
\hline Totals & 32 & 24 & 23 \\
\hline
\end{tabular}

sources, coincidence counting, delayed-neutron measurements, photoneutron interrogation, and neutron generators. Fig. 24 shows participants working on the photoneutron experiment during the course. Because of the increased use of neutron NDA planned for the SRP, a large number of duPont employees attended the 1983 course.

The gamma-ray course emphasizes the use of highresolution gamma-ray spectroscopy systems using germanium detectors in applications such as uranium and plutorium isotopic measurements, segmented gamma scanning, absorption-edge densitometry, and $x-$ ray fluorescence. Figure 25 shows participants working on the uranium enrichment experiment during the course. Because gamma-ray techniques are widely used for both portable and in-plant measurements, this course has been particularly useful to DOE facility operators and NRC and IAEA inspectors.

In addition to the three regular training courses, Los Alamos was responsible for two training seminars in

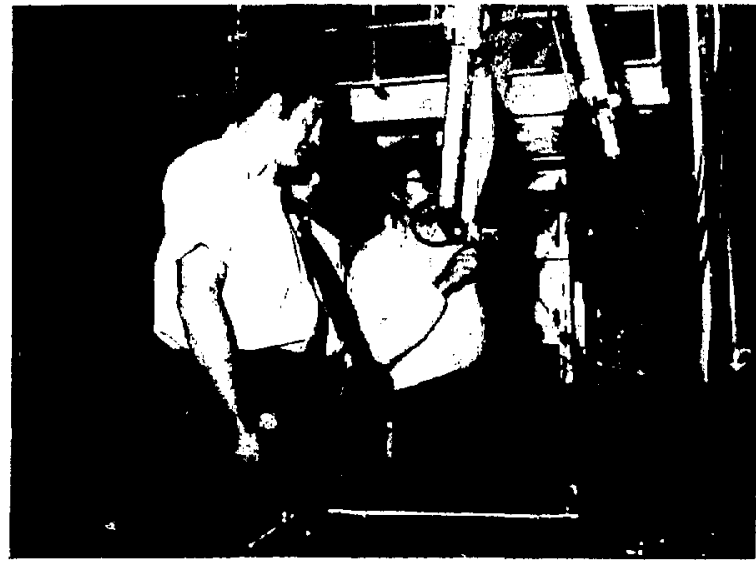

Fig. 24. Participants observe Los Alamos instructor using remote manipulator to move intense photoneutron source during the June 1983 course, Neutron Assay of Nuclear Material. 


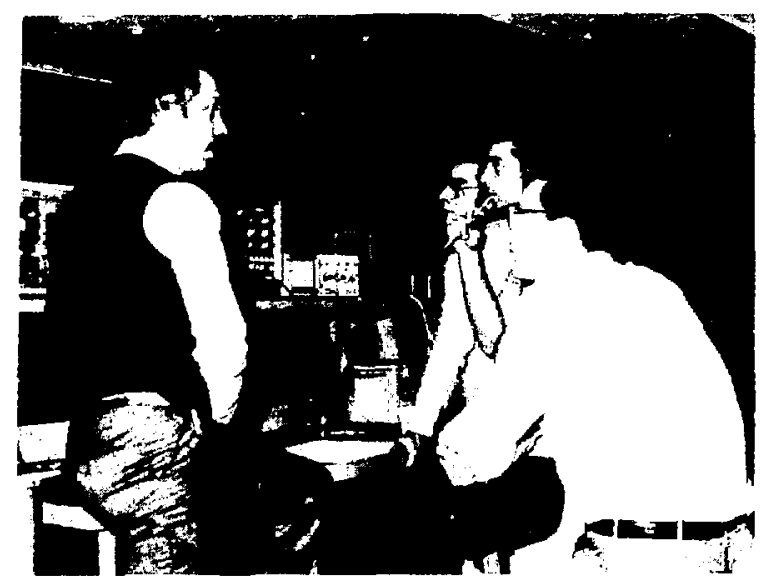

Fig. 25. Participants discuss uranium enrichment measurement with a portable MCA during the December 1983 course. Gamma-Ray Assay of Nuclear Material.

1983. A workshop on plutonium NDA technology, which was held here on March 8 and 9, 1983, was attended by 22 people from the Rockwell facility at Rocky Flats, Mound Laboratory, Albuquerque Operations Office, and Los Alamos National Laboratory. Los Alamos also participated with the DOE Oak Ridge Operations Office (DOE/OR) in planning and organi:ing a workshop on NDA for enrichment facilities. This workshop, which was held September 21 and 22, 1983, in Oak Ridge, was attended by 50 people from DOE enrichment facilities in Oak Ridge, Portsmouth, and Paducah, as well as from DOE/OR, Los Alamcs, Oak Ridge National Laboratory, Y-12, National Lead, Mound Laboratory, and Oak Ridge Associated Universities. Five people from Los Alamos attended the workshop and gave technical presentations.
The 1984 DOE Safeguards Technology Training Program schedule includes the following three courses.

- Materials Accounting for Nuclear Safeguards, April 1984

- Fundamentals of Nondestructive Assay of Nuclear Material, October 1984

- Gamma-Ray Assay of Nuclear Material, December 1984

The development, during 1984, of a new course on attribute measurement is under serious consideration. This course would be presented during 1985 and would deal with rapid measurement techniques applied to large inventories and materials holdup in process equipment. 


\section{PART 2: SECURITY DEVELOPMENT AND SUPPORT}

\section{GOMPUTER SECURITY}

\section{A. Center for Computer Security (D. G. Harder, OS-4)}

The Center continues to be involved heavily in DOE computer security activities. The Center published a 56page summary of the activities of the 5th Computer Security Group Conference held in Knoxville, Tennessee, in November of 1982. This newsletter was mailed to more than 550 recipients. Center personne! also were very active in the preparation and planning for the 6th Computer Security Group Conference held in Denver, Colorado, July 26-28, 1983. In coordination with the Program Chairman and Conference Host, Center personnel produced and mailed conference invitations, agenda, and registration forms, and also were responsible for preregistration of attendees and ensuring smooth operation of the conference activities. Center personnel provided session chairmen and speakers for the conference. Center personnel also summarized the papers given at this 6 th Conference and produced a document comprising these summaries and other conference proceedings. Based on the experience at the 6 th DOE Computer Security Group Conference, we proposed a number of changes that would allow us to improve and expand our services to the DOE Computer Security Group. Many of these suggestions were accepted by the Steering Committee of the Computer Security Group and will allow the Center to be more responsive to the needs of the computer-security community.

We also produced a November newsletter that was mailed to more than 700 recipients. This large number of recipients is indicative of the importance placed on computer security not only by the DOE but also by other organizations and the value of an organization such as the Center.

We began work late this year on a class for Computer System Security Officers (CSSOs). Training of CSSOs is central to computer security within the DOE complex. The syllabus for this class has been approved by various interested parties in both DOE Headquarters and area offices. We hope that this class, which is scheduled for several presentations in 1984, will satisfy the need for consistent and comprehensive education of CSSOs within the DOE family.

To provide more timely and high-quality service for its publication needs, the Center for Computer Security has installed a laser printer. It is connected to the Digital Equipment Corporation VAX 11/750 used by the Center.

\section{B. Operational Model (OPMODEL) Support (D. G. Harder and R. E. Lewis, OS-4)}

Center personnel, Ed Springer and Duane Harder, each served as chairman of the technical support subpanel of the security panel of the DOE's Telecommunications OPMODEL Group (DETOG). Others provided support to the project on a part-time basis by performing investigations, supplying expertise, and serving on panels and subpanels as needed. Several documents, provided to Headquarters in draft form, addressed the security requirements necessary for $O P$ MODEL Phase 1 (security requirements necessary for interconnecting the first four nodes) and security requirements during the network phase (connection of more than the initial four nodes). In a paper entitled "Some Comments on Security Policies, Protecting Data Flows Between Open and Closed Partitions," we pointed out that an extremely stringent security policy induces high economic costs that are probably intolerable for most OPMODEL sites. We discussed requiring all OPMODEL sites to write and maintain software that runs in various partitions and evaluated the possibility of using externally written software in DOE computer facilities. We also addressed the necessity of limiting sabotage potential within software packages used within OPMODEL.

The Center continues to be involved in the X.25 assessment effort by maintaining contact with manufacturers concerning their $\mathrm{X} .25$ support and investigating OPMODEL requirements at various potential OPMODEL sites.

To minimize the cost of OPMODEL, we investigated the possibility of using the Honeywell Secure Communications Processor (SCOMP) as a secure gateway, or front end, at each node. This possibility was explored at length and presented to senior OPMODEL technical personnel. That briefing resulted in determining a different approach to defining security requirements for subsequent phases of the OPMODEL project beyond the initial four node installations.

A paper entitled "Audit Trails on Threat Management," which examines the role of audit trails in OPMODEL, was prepared for the security panel. 
Continued study of security problems associated with OPMODEL resulted in a proposal for a joint experiment with the Sandia National Laboratories, Albuquerque. This project will investigate the feasibility and requirements of a secure wide-band communication network that would connect various DOE sites. We expect this effort to culminate in a network connecting these sites in a manner that allows them to transmit large volumes of classified data at high speeds.

\section{Secure Computer Systems (E. A. Springer, E-8)}

The SCOMP system was placed in operation in the Center in January. At that time, the requirements document describing how the SCOMP could be used as a security controller in the General Electric (GE) Neutron Division at Pinellas, Florida, was completed, as were the system specification documents. Source code for the SCOMP and for the remote terminal emulator were made available by the manufacturer in March and provided additional capability for debugging and testing the system. Early in the year, the hardware was relatively unstable, as might be expected in a developmental machine. Because of the number of hardware and software fixes that were required, GE reduced its effort in this task to a role of consulting with Los Alamos personnel. This encouraged us to learn the current state of the art with regard to secure computer systems and their application in secure front-end systems.

As part of the Center's activities, we cooperated with the Department of Defense (DoD) Center for Computer Security in the evaluation of the SCOMP. The DoD team leader incorporated two Los Alamos reports in his evaluation. Those reports discussed trusted start-up and recovery procedures and testing of trusted software. The SCOMP system is now a fully supported product of the Honeywell Corporation, which implies that we should now be able to perform the throughput and reliability testing we would on a mature system. Preliminary throughput tests were begun in early October, and the SCOMP test and evaluation is continuing. The final machine structure is now understood and files can be used effectively. A software coupler, which will allow the SCOMP to be linked to a larger Honeywell mainframe, has been installed at GE, Pinellas, and will be tested soon.

Although the formal joint project between GE and Los Alamos has terminated, we will continue to use GE as consulting engineers, and the software and technology developed by GE will be used at Los Alamos for continued SCOMP testing and evaluation.
We anticipate that we will be able to thoroughly test throughput, reliabiiity, and other features of the secure communications processor in 1984 .

\section{Computer and Network Access Controls (D. P. Brenner, S. M. Mniszewski, and E. A. Springer, E-8)}

1. Access Control and Encryption (ACE) System. We are continuing development of the LINK ACE system at Los Alamos. This system is designed to protect unclassified sensitive data transmissions over unprotected lines and between computers and remote terminals. This project is intended to fill a need within the DOE not yet satisfied by commercial products. Although there is some commercial activity, no equipment is available that meets DOE requirements. Primary activity this year has centered on refining the developmental system that was demonstrated earlier and in incorporating suggestions made as the result of use of the prototype system.

The LINK/ACE system has now been installed in an operational test bed on the DOE Central Personnel Clearance Index (CPCI) system. This system is a clearance information data base network based in Germantown, Maryland, with remote terminal points in different area offices. The master LINK/ACE unit has been installed on the computer in Germantown. Remote LINK/ACE units have been installed in ten area offices: Schenectady, Pittsburgh, Chicago, Richland, Idaho Falls, San Francisco, Las Vegas, Albuquerque, Oak Ridge, and Savannah River. Both master and remote LINK/ACE units are installed in physically protected environments but use dial-up telephone lines for communications. Both hardware and operations have been approved by the National Security Agency for this system based on Federal Standards 1026 and 1027. The master LINK/ACE and the remote LINK/ACE are functionally identical, and the encryption units are commercially available, thus providing a means to lower overall cost and reduce maintenance requirements.

Technology developed for LINK/ACE can be applied in many situations for the protection of unclassified, sensitive information that must be transmitted by the Government. It provides both communications security and user authentication. The encryption method designed into the LINK/ACE prevents both passive and active line taps, and useful information cannot be received from, or transmitted to, the communication link unless the user has the appropriate LINK/ACE unit. 
2. Distributed System Security. After delivery of a prototype encryption unit, SNAP, developed by Associated Computer Consultants (ACC) of Santa Barbara, LLNL became a beta test site for the device, which is based on a Motorola MC68000 processor. LLNL suffered through the normal problems associated with being a beta test site and continued to work with ACC on capability protection software in the SNAP unit. The Center designed and implemented the VAX/VMS software for testing the capability protection performance of the SNAP unit.

Testing the SNAP unit demonstrated its feasibility and pointed out needed improvements, which were related primarily to the response time of the unit. Changes made by ACC in the unit's microcode and communications techniques remedied the problem. (During this time, the ACC personnel were incorporated, calling themselves the Xypher Security Systems Group.)

The beta test site report on the SNAP init at Livermore was prepared and delivered to $\mathrm{XY}$. .. tems by the Center, and at that time Xypher Systems ac. ta new unit that corrected the deficiencies found in beta site testing.

Because of our interaction with the vendor in acting as a beta test site, much valuable information was gained for the DOE, and input was delivered to the vendor, which encouraged the vendor to incorporate the changes of interest to the DOE.

\section{E. Management Support Tools (S. T. Smith, Q-4)}

The activities covered in this section were not supported by Security Development and Support funds, but a requirement for the activity described here exists at the Laboratory. Because the efforts expended to satisfy that requirement are appropriate for this section and are of interest to the readers of this report, they are presented here.

Proper management of a computing center or facility requires analyzing risks and vulnerabilities of the facility. However, heretofore, attempts at risk analysis and vulnerability studies of computer facilities have not been well received. The risk analysis methodology described here is both accurate and understandable and is based on substantial theoretical investigations, whereas earlier risk analysis methodology demanded a large amount of difficult-to-obtain information. The present method is based on a linguistic algebra and determines realistic accurate, and qualitative as (opposed to quantitative) measures of the impact and risk of identified vulnerabilities. It uses an interactive conversational questionnaire that is straightforward and easily used even by those without previous computer experience.

The present prototype of the final system demonstrates that the concept is valid and can be implemented efficiently. In this prototype, natural hazards and direct human threats are evaluated against a complete set of targets; the indirect human threats are not yet implemented. The prototype system is undergoing beta testing at several sites.

Plans include developing a full working system that addresses all three kinds of threat. Refinements necessary for more convenient use of the package will be added. These include graphical and tabular displays of results, automated summary reports, and a fully automated feedback system that will permit the user to model different scenarios in an interactive mode.

The methodology promises to be valuable not only because is it applicable to computer facilities, but also vecause the technique and the methodology can be extended or modified readily for many other applications, such as identifying vulnerabilities in the nuclear data communications systems of power control rooms, process-control computers and systems, and electrical power switching systenus or power generation or distribution facilities.

\section{OPERATIONAL SECURITY (OPSEC) AND VULNERABILITY ASSESSMENTS (VAS) (R. W. Wagner and T. D. Bearce, OS-1)}

Vulnerability Assessments, which were begun last year, were improved and continued. They were conducted by personnel from Los Alamos and the Freeze Corporation. Typically a VA is coordinated with DOE Headquarters and the facility to be assessed, and, following the assessment, an exit briefing is given to members of staffs of DOE Headquarters, the appropriate DOE area office, the site receiving the VA, and other interested parties. Five VAs have been completed. The report for the first VA, of the PANTEX Plant, was published in late January 1983. The report on the SRP VA, which was conducted in November 1982, was published in March 1983. The Rocky Flats Plant VA, which was completed February 7, 1983, was published in final form in April. The 4th OPSEC VA, that of the Transportation Safeguards Division Albuquerque, was completed in late April 1983 and published in late June. In September 1983, an OPSEC VA was conducted at the UCC/ND Y-12 Plant Complex. A final report on this OPSEC VA has not been published; DOE authorization is pending. 
OPSEC support also was provided at the DOE exercise Equus Red. A draft copy of the results of that support has been given to the coordinator of the Equus Red exercise.

A preliminary visit has been paid to the proposed site of the 6th OPSEC VA.

The DOE OPSEC procedural guide has undergone several revisions during the year as comments and suggestions from DOE Headquarters, Operations and Area Offices, and contractors have been incorporated.
The guide presently comprises two volumes. Volume l, including programs and procedures, was printed, bound, and distributed to DOE field offices in January 1984. A final draft of Volume II has been prepared and submitted to DOE/OSS for comment.

Conference participation of note includes that of the 3rd Annual DOE OPSEC Conference in October, at the Nevada Operations Office. Papers were presented by Los Alamos personnel and the Freeze Corporation. 


\section{PART 3: SAFEGUARDS TECHNOLOGY DEVELOPMENT}

\section{EQUIPMENT DEVELOPMENT AND ENGI- NEERING}

\section{A. NDA Technology Development}

1. End-Crop Box Counter (J. K. Sprinkle, Jr., J. E. Stewart, C. O. Shonrock, K. E. Kronke, J. N. Leavitt, H. R. Dye, and J. Baca, Q-1). The United Nuclear Corporation (UNC) operates the N Reactor near Richland, Washington, to produce electricity for the Northwest power grid and plutonium for the DOE. The UNC also manufactures the uranium-metal fuel for this reactor using an extrusion process. The ends of the extrusions are cut off because a nonuniform uranium concentration prohibits their use as fuel, an 1 these "end crops" are shipped off site to be dissolved and recycled. Up to 12 end crops can be generated each hour. They usually are shipped in a box containing up to 50 end crops and weighing as much as $544 \mathrm{~kg}$. In the past they were transferred on the basis of a by-difference number, which led to some significant shipper-receiver differences. Los Alamos was asked to provide a measurement system at UNC that could improve uranium accountability while having minimal impact on facility operations.
We designed an instrument ${ }^{24}$ that measures neutrons from the spontaneous fission decay of ${ }^{238} U$ in the fuel material (Fig. 26). This instrument was installed at UNC in September 1982. In 1983, UNC collected extensive data that were used to generate the instrument calibration. The UNC then measured secondary standards to confirm this calibration..$^{25}$ Before accepting the instrument, UNC noted a few per cent discrepancy with their by-difference estimates. The NDA values were confirmed by measuring uranium billets of known mass and by dissolving several boxes of end crops and measuring the contained uranium with destructive assay techniques. Table $\mathrm{V}$ shows the results of these tests. The verification exercises indicated a bias of approximately $1 \%$ in the counter results, which was well within the design goal of $2 \%$. However, further investigation at UNC showed that the original calibration weights were incorrectly reported by $1 \%$. The calibration will be improved in 1984, and further dissolution experiments also are being considered. The instrument now has been accepted as the accountability device for end-crop shipments, and UNC is considering using it also for process control. In addition, the instrument may be used to measure finished fuel shipments if the size of the shipping crate can be reduced.

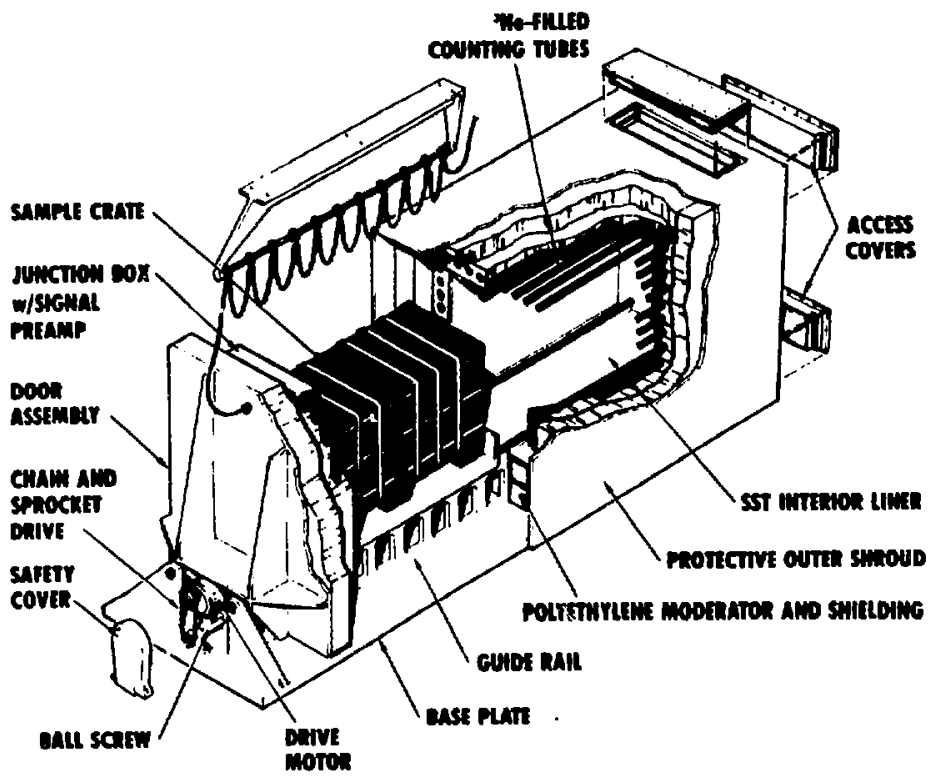

Fig. 26. Conceptual design of the end-crop box counter. 


\begin{tabular}{lccc}
\hline \hline TABLE V. $\begin{array}{l}\text { End-Crop Measurement Verification } \\
\text { Results }\end{array}$ & \multicolumn{2}{c}{ Mass (kg) } \\
\cline { 3 - 4 } & & & \\
& & & \\
& By-Difference & NDA & Recovered \\
\hline & & & \\
Dissolved & 223.8 & 200.3 & 202.9 \\
End-Crop & 184.6 & 163.2 & 165.7 \\
Shipments & 241.0 & 226.7 & 229.6 \\
& 217.7 & 195.3 & 198.3 \\
& 185.8 & 162.0 & 164.5 \\
& 199.4 & 177.1 & 179.4 \\
& & & \\
Feed Stock & & & Known \\
Billets & & 128.0 & 128.4 \\
\hline \hline
\end{tabular}

2. Determination of Plutonium Isotopic Ratios Using Low-Energy Gamma-Ray Spectroscopy (T. K.Li, Q-1). The measurement of plutonium isotopic composition is important for nuclear material accountability, and much effort has been devoted to the development of gamma-ray spectroscopy techniques for this measurement. ${ }^{26-28}$ Whereas gamma rays above $100 \mathrm{keV}$.are used successfully to measure ${ }^{239} \mathrm{Pu}$ and ${ }^{241} \mathrm{Pu}$, measurements in this energy range give ${ }^{238} \mathrm{Pu}$ and ${ }^{240} \mathrm{Pu}$ precisions of only 2 to $4 \%$ for count times of $14 \mathrm{~h}$ and sample masses of $0.25 \mathrm{~g}$. We are developing a method to improve the accuracy and precision of plutonium isotopic measurements. ${ }^{29,30}$

The approach is to analyze the high-intensity gamma rays at $43.48,45.23,51.63,59.54$, and $64.83 \mathrm{keV}$ from ${ }^{238} \mathrm{Pu},{ }^{240} \mathrm{Pu},{ }^{239} \mathrm{Pu},{ }^{241} \mathrm{Am}$, and ${ }^{241} \mathrm{Pu}$. The gamma rays below $59.54 \mathrm{keV}$ are difficult to measure in aged samples due to strong interference from the Compton continuum of the very intense $59.54-\mathrm{keV}$ gamma ray from ${ }^{241} \mathrm{Am}$ and ${ }^{237} \mathrm{U}$; therefore, this low-energy region has been used previously only for the measurement of freshly separated solutions. ${ }^{28}$ However, our study indicates that this difficulty can be reduced by selecting a detector with the proper combination of resolution, efficiency, and peak-to-Compton ratio at energies below $60 \mathrm{keV}$.

All gamma-ray peak areas are calculated using a channel summation method with a linear background subtraction. Minor interferences in the full energy peaks are taken into account. We calculate the relative efficiency of gamma-ray detection from each sample using the measured peak area and known specific activity of ${ }^{239} \mathrm{Pu}$ gamma rays at $38.66,51.63$, and $68.72 \mathrm{keV}$ in the spectrum under study. Intermediate efficiency values are found by logarithmic interpolation.

An instrument that uses this analytical technique has been installed at the Los Alamos Plutonium. Processing Facility (Fig. 27), and aged plutonium samples in several chemical formis have been analyzed. The isotopic composition of these samples varied from 82 to $98 \%$ ${ }^{239} \mathrm{Pu}$, and sample mass varied from $10 \mu \mathrm{g}$ to $4 \mathrm{~g}$ plutonium. The americium content was as high as 3390 $\mu \mathrm{g} \mathrm{Am} / \mathrm{g}$ Pu. Table VI compares preliminary gammaray spectroscopy results (20000-s count time) with mass spectrometry analysis $\left({ }^{238} \mathrm{Pu}\right.$ and ${ }^{241} \mathrm{Am}$ determined by radioanalysis). These measurements show negligible bias, and the overall precision of the technique is indicated by the $1.4 \%$ standard deviation of the ${ }^{240} \mathrm{Pu} /{ }^{239} \mathrm{Pu}$ ratio. The larger standard deviation of the ${ }^{241} \mathrm{Pu} /{ }^{239} \mathrm{Pu}$ ratio (6.3\%) is caused by the low intensity of 64.8-keV gamma rays and the low ${ }^{241} \mathrm{Pu}$ isotopic abundance in some samples. The standard deviations $(4.6 \%$ and $5.6 \%$ ) of the ${ }^{238} \mathrm{Pu} /{ }^{239} \mathrm{Pu}$ and ${ }^{241} \mathrm{Am} /{ }^{239} \mathrm{Pu}$ ratios may reflect the uncertainties of radioanalysis.

In Table VII, the estimated precisions from lowenergy gamma-ray spectroscopy are compared with those from higher-energy gamma-ray spectroscopy (120 to $160 \mathrm{keV}$ ) for a 20000 -s count time. The low-energy region gives better precisions for all isotopic ratios within the plutonium mass range $30 \mu \mathrm{g}$ to $4 \mathrm{~g}$.

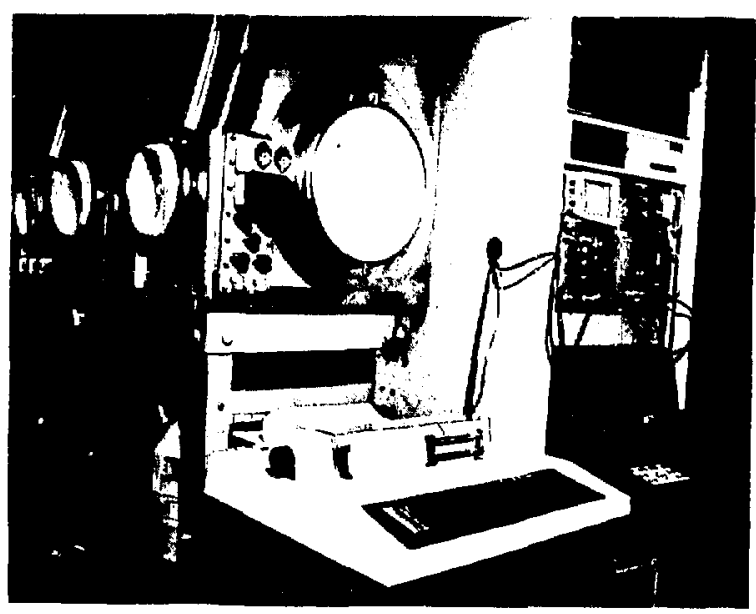

Fig. 27. The low-cnergy plutonium isotopic composition analyzer installed under a glove box at the Los Alamos plutoniun facility. 


\begin{tabular}{|c|c|c|c|c|c|c|c|c|}
\hline \multirow[b]{2}{*}{ Sample } & \multirow{2}{*}{$\begin{array}{c}\text { Pu } \\
\text { Mass }\end{array}$} & \multirow[b]{2}{*}{$\begin{array}{c}\text { Chemical } \\
\text { Form }\end{array}$} & \multirow{2}{*}{$\begin{array}{c}\mathrm{Am} / \mathrm{Pu} \\
(\mathrm{ppm})\end{array}$} & \multirow{2}{*}{$\begin{array}{c}{ }^{240} \mathrm{Pu} \\
(\%)\end{array}$} & \multirow{2}{*}{$\begin{array}{c}\text { Ratio: } \\
{ }^{8 \mathrm{Pu} /{ }^{239} \mathrm{Pu}}\end{array}$} & \multicolumn{2}{|c|}{$\begin{array}{l}\text { Gamma Spectroscopy } \\
\text { Mass Spectrometry }\end{array}$} & \multirow[b]{2}{*}{${ }^{241} \mathrm{Am} /{ }^{239} \mathrm{Pu}$} \\
\hline & & & & & & ${ }^{240} \mathrm{Pu} /{ }^{239} \mathrm{Pu}$ & ${ }^{241} \mathrm{Pu} /{ }^{239} \mathrm{Pu}$ & \\
\hline $\begin{array}{l}\text { MS 10 } \\
\text { MS 30 } \\
\text { MS 600 } \\
\text { ST 121 } \\
\text { ST 119 } \\
\text { ST 151 } \\
\text { SOL 01 } \\
\text { SOL 05 } \\
\text { SOL 06 } \\
\text { JTO 02 }\end{array}$ & $\begin{array}{c}10 \mu \mathrm{g} \\
30 \mu \mathrm{g} \\
600 \mu \mathrm{g} \\
4 \mathrm{~g} \\
1.72 \mathrm{~g} \\
2.65 \mathrm{~g} \\
0.2 \mathrm{~g} \\
0.2 \mathrm{~g} \\
0.2 \mathrm{~g} \\
2.58 \mathrm{~g}\end{array}$ & $\begin{array}{l}\text { Fluoride } \\
\text { Fluoride } \\
\text { Fluoride } \\
\text { Oxide } \\
\text { Oxide } \\
\text { Oxide } \\
\text { Nitrate } \\
\text { Nitrate } \\
\text { Nitrate } \\
\text { Metal }\end{array}$ & $\begin{array}{r}3390 \\
96 \\
372 \\
1864 \\
2220 \\
40 \\
166 \\
1514 \\
1500 \\
1800 \\
838\end{array}$ & \begin{tabular}{|r|r|}
6.64 \\
11.40 \\
6.01 \\
16.45 \\
11.76 \\
2.01 \\
6.19 \\
14.04 \\
16.45 \\
5.94
\end{tabular} & $\begin{array}{l}0.941 \pm 0.047^{b} \\
1.002 \pm 0.033 \\
0.952 \pm 0.006 \\
0.984 \pm 0.036 \\
1.082 \pm 0.035 \\
1.041 \pm 0.030 \\
1.067 \pm 0.007 \\
0.990 \pm 0.015 \\
0.997 \pm 0.017 \\
0.983 \pm 0.029\end{array}$ & $\begin{array}{l}1.038 \pm 0.054 \\
0.997 \pm 0.016 \\
1.001 \pm 0.007 \\
1.005 \pm 0.025 \\
1.008 \pm 0.025 \\
0.993 \pm 0.004 \\
0.997 \pm 0.002 \\
0.989 \pm 0.011 \\
1.009 \pm 0.012 \\
0.991 \pm 0.017\end{array}$ & $\begin{array}{l}1.038 \pm 0.192 \\
0.967 \pm \mathbf{0 . 1 3 0} \\
\mathbf{0 . 9 9 9} \pm \mathbf{0 . 0 4 2} \\
\mathbf{0 . 9 9 1} \pm \mathbf{0 . 0 6 1} \\
\mathbf{0 . 9 3 0} \pm \mathbf{0 . 0 4 2} \\
\mathbf{1 . 0 8 2} \pm \mathbf{0 . 2 0 0} \\
\mathbf{1 . 1 0 7} \pm \mathbf{0 . 0 2 3} \\
\mathbf{0 . 9 2 9} \pm \mathbf{0 . 0 4 5} \\
1.043 \pm \mathbf{0 . 0 4 8} \\
\mathbf{0 . 9 4 7} \pm \mathbf{0 . 0 8 2}\end{array}$ & $\begin{array}{c}- \\
- \\
- \\
0.993 \pm 0.026 \\
0.927 \pm 0.016 \\
0.936 \pm \mathbf{0 . 0 0 2} \\
1.051 \pm 0.004 \\
1.017 \pm 0.019 \\
1.065 \pm \mathbf{0 . 0 2 4} \\
1.048 \pm 0.008\end{array}$ \\
\hline \multicolumn{2}{|c|}{ Average } & & & & 1.004 & 1.003 & 1.003 & 1.005 \\
\hline \multicolumn{3}{|c|}{ Standard Deviation } & & & 0.046 & 0.014 & 0.063 & 0.056 \\
\hline
\end{tabular}

Plutonium-238 and ${ }^{241} \mathrm{Am}$ determination by radioanalysis.

bucertainties represent the precision (1 $\sigma)$ of gamma-ray spectroscopy as estimated from counting statistics, including uncertainties from relative efficiencies.

\begin{tabular}{|c|c|c|c|c|c|c|c|c|c|}
\hline \multirow[b]{2}{*}{ Sample } & \multirow[b]{2}{*}{$\begin{array}{c}\text { Pu } \\
\text { Mass }\end{array}$} & \multicolumn{2}{|c|}{${ }^{238} \mathrm{Pu} /{ }^{239} \mathrm{Pu}$} & \multicolumn{2}{|c|}{${ }^{240} \mathrm{Pu} /{ }^{239} \mathrm{Pu}$} & \multicolumn{2}{|c|}{${ }^{241} \mathrm{Pu} /{ }^{239} \mathrm{Pu}$} & \multicolumn{2}{|c|}{${ }^{241} \mathrm{Pu} /{ }^{239} \mathrm{Pu}$} \\
\hline & & $\begin{array}{l}43.5 / \\
51.6\end{array}$ & $\begin{array}{l}152.7 / \\
129.3\end{array}$ & $\begin{array}{l}45.2 / \\
51.6 \\
\end{array}$ & $\begin{array}{l}160.3 / \\
129.3 \\
\end{array}$ & $\begin{array}{l}64.8 / \\
51.6 \\
\end{array}$ & $\begin{array}{l}148.6 / \\
129.3\end{array}$ & $\begin{array}{l}59.5 / \\
51.6 \\
\end{array}$ & $\begin{array}{l}125.3 / \\
129.3 \\
\end{array}$ \\
\hline IS 30 & $30 \mu \mathrm{g}$ & 3.3 & 37.8 & 1.6 & 36.8 & 13.0 & 21.5 & 6.3 & 39.0 \\
\hline MS 600 & $600 \mu g$ & 0.6 & 10.0 & 0.7 & 13.0 & 5.2 & 7.3 & 1.3 & 8.4 \\
\hline OL 05 & $0.2 \mathrm{~g}$ & 1.5 & 14.1 & 1.1 & 18.9 & 4.5 & 9.7 & 1.9 & 3.6 \\
\hline ST 121 & $4 \mathrm{~g}$ & 3.6 & 9.6 & 2.5 & 10.0 & 6.1 & 6.4 & 2.6 & 9.1 \\
\hline
\end{tabular}

These results demonstrate that the low-energy plutonium isotopic analysis technique can measure moderately aged plutonium samples in a wide range of mass, isotopic content, and chemical form. Because the technique can be used to measure samples in the microgram mass range, it may be ideal for measurement of resin beads used by the IAEA to send plutonium samples to Vienna for analysis. We are investigating this potential application.
3. Highly Accurate Gamma-Ray Assuy of ${ }^{235} U$ in Solution (J. L. Parker, Q-1). Nondestructive assay has become an important part of the safeguards procedures used to protect nuclear materials, and for more than a decade gamma-ray spectrometric measurements have been used for safeguards, process control, quality control, and waste management. Obviously, more accurate nondestructive measurement systems are more sensitive in detecting possible nuclear material 
diversions. Improved accuracy also contributes directly to better process control, quality control, and waste management. Because uniform, homogeneous solutions offer the possibility of highly accurate nondestructive gamma-ray assays, a new task to develop a system to measure the ${ }^{235} \mathrm{U}$ content of enriched uranium solutions has provided the opportunity to study some of the important factors influencing the accuracy of quantitative gamma-ray assay.

Design goals for the system require the determination of the ${ }^{235} \mathrm{U}$ mass in $30-\mathrm{m} \ell$ samples with concentrations ranging from $I$ to $500 \mathrm{~g} \mathrm{U} / \ell$ with accuracies as close to $0.1 \%$ as possible. To achieve or even closely approach that goal will represent a significant advance over previous systems. Measurements are based on the detection of the $186-\mathrm{keV}$ gamma ray emitted by ${ }^{235} \mathrm{U}$ at a rate of 43000 per second per gram.

The accuracy-related factors that we have examined are the long-term stability of the counting equipment, information losses caused by pulse pileup and electronic deadtime, losses caused by self-absorption of gamma rays within the samples, and errors caused by variation in the dimensions of the sample bottles. Computational and experimental studies during the past year indicate that all the factors can be controlled or corrected with accuracies near $0.1 \%$. For example, Table VIII summarizes the results of an experimental study of the accuracy with which information losses caused by pulse pileup and electronic deadtime can be corrected for count rates between 16000 and 60000 events per second. Additional results of these studies were in- cluded in a recent paper presented at the INMM/ANS topical meeting on the Process-Safeguards Interface. ${ }^{22}$

The assay-system development is now approaching the stage where a prototype will test the ability to control all the factors simultaneously. Experiments during FY 1984 will determine whether the practical limit of accuracy will be one-tenth of a per cent or several tenths of a per cent. Although these studies are being conducted for a specific instrument task, the results will apply to all future gamma-ray assay systems.

4. K-Edge Plutonium Solution Densitometer (H. A. Smith, Q-1). In FY 1982 we installed a K-edge densitometer at SRP to measure the plutonium concentration in the precipitator feed solution of the FB-process line. The subsequent test and evaluation program was nearly completed during 1983. The instrument (Fig. 28) measures the differential absorption by the sample solution of gamma radiation from selected radioactive sources. The operation and data analysis are carried out under computer control. Assay precisions of approximately $0.5 \%$ have been achieved on $30 \mathrm{~g} \mathrm{Pu} / \ell$ solutions in off-line tests. On-line measurements have recently been completed on approximately 30 samples, and preliminary analysis indicates good instrument performance under actual process conditions. These results will be analyzed and reported during FY 1984.

This program has demonstrated the feasibility of using on-line nondestructive assay techniques to obtain timely, high-quality accountability data. Assay results

\begin{tabular}{|c|c|c|}
\hline TABLE VIII. & $\begin{array}{l}\text { Results of } \\
\text { ence Source } \\
\text { Correction } \\
\text { Losses Cau } \\
\text { time and Pil }\end{array}$ & $\begin{array}{l}\text { est of Refer- } \\
\text { Procedure for } \\
\text { of Counting } \\
\text { ed by Dead- } \\
\text { up }\end{array}$ \\
\hline $\begin{array}{l}\text { Approximate } \\
\text { Count Rate } \\
\text { (counts/s) }\end{array}$ & $\begin{array}{c}\text { Total } \\
\text { Correction } \\
\text { Required } \\
(\%)\end{array}$ & $\begin{array}{l}\text { Accuracy of } \\
\text { Correction } \\
(\%)\end{array}$ \\
\hline 60000 & 168 & 0.10 \\
\hline 53200 & 143 & 0.03 \\
\hline 46300 & 119 & 0.01 \\
\hline 39900 & 98 & 0.03 \\
\hline 33700 & 80 & 0.03 \\
\hline 27300 & 62 & 0.04 \\
\hline 16000 & 35 & 0.02 \\
\hline
\end{tabular}

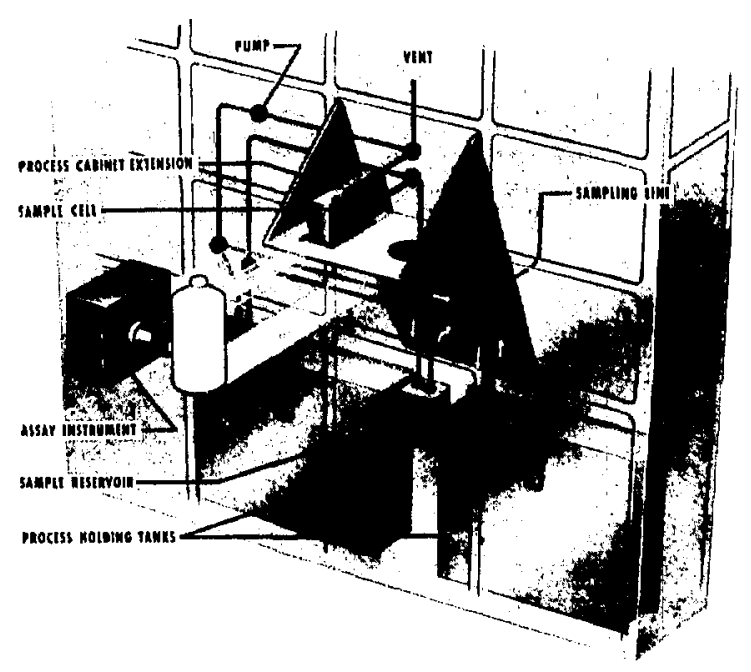

Fig. 28. A schematic of the K-edge densitometer installed at the SRP. 
were available in 30 to $40 \mathrm{~min}$ and did not require the removal of an accountability sample or a time-consuming chemical analysis. The test and evaluation exercise will be concluded by documenting the study results and suggested improvements to the measurement procedures and computer software. The instrument also may be tested at other key measurement points in the process.

5. Evaluation and Calibration of the Los Alamos LEdge Densitometer (P. A. Russo, D. G. Langner, and J. K. Sprinkle, Q-1). The final report of the evaluation of the Los Alamos L-edge densitometer at the DOE's New Brunswick Laboratory (NBL) was published in July $1983,{ }^{31}$ and some examples of the evaluation data were published previously. ${ }^{32}$ This evaluation included extensive analysis of the L-edge assay results on prepared reference solutions of uranium and plutonium. It covered a range of SNM concentrations $(5$ to $80 \mathrm{~g} / \mathrm{l}$ ) and uranium-to-plutonium ratios ( 1 to 5 ) and included special solution assays for which the reference materials were "spiked" with i $0 \%$ (by weight) impurities of low-, intermediate-, and high- $Z$ elements.

The precision (RSD) of the L-edge assay results for the single SNM component (uranium or plutonium) solutions is better than $0.5 \%$ for 1000 -s assays of solutions with concentrations in excess of $20 \mathrm{~g} / \mathrm{l}$. The bias in the assay of these solutions is less than $0.3 \%(1 \sigma)$. For the assay of mixed uranium and plutonium solutions, the RSDs (for concentrations greater than $20 \mathrm{~g} / \mathrm{l}$ ) are better than $0.5 \%$ and $0.9 \%$, respectively. These results are independent of impurities present in the spiked reference solutions.

The evaluation performed at NBL is considered exemplary for several reasons.

- The precision and accuracy of the L-edge assay determined in this evaluation were shown to be independent of any significant (random or systematic) effects caused by sampling or by uncertainties in the reference values. This was made possible by the quality of the destructive analytical chemistry at NBL and by strict adherence to a protocol for measurement control (developed at NBL specifically for this evaluation) in obtaining and using the destructive analytical results.

- Precisely characterized reference materials spanning the entire useful range of this instrument for uranium, plutonium, and mixed (uranium and plutonium) solutions with and without impurities were prepared and used, within only a few months, to acquire the complete set of evaluation data.
- Development and execution of the procedures for evaluation of the L-edge instrument were carried out entirely by NBL personnel and independently of the Los Alamos instrument developers. Collaboration during the evaluation period was limited to exchangc of user information on instrument operation.

The L-edge densitometer has been returned to Los Alamos, and we are attempting to identify a suitable location for in-plant operational evaluation of this instrument.

6. Plutonium Isotopics by Gamma-Ray Spectroscopy (T. E. Sampson and S. -T. Hsue, Q-1). In FY 1982 we developed and shipped instrumentation to the SRP to measure the isotopic composition of the solid plutonium product of the FB-process line. This information is combined with calorimetry measurements of the same material to give total plutonium content. The instrument employs high-resolution gamma-ray spectroscepy and computer-based data acquisition and analysis. It was first turned on at SRP approximately 2 years after shipment. Precision tests showed excellent agreement with the performance demonstrated in the laboratory (about $0.8 \%$ RSD for the specific power from a 1-h assay). The instrument accuracy is now being tested by comparing its assay results with mass spectrometric measurements.

A computer-based instrument of this type can provide high-quality assay results on final product inventory in a comparatively short time ( 1 to $2 \mathrm{~h}$ ) without invasion of the product material. Current product analysis involves the off-line destructive assay of a sample removed from every product item.

After conclusion of the present tests, the instrument may be accepted for routine product accountability measurements.

7. Flowing Fissile Assay System (L. G. Speir and E. L. Adams, Q-1). We are developing a method to measure mass flow rates and nuclear materials concentrations in the solution streams of nuclear processing plants (Fig. 29). An assay is accomplished by pumping the solution through an irradiation chamber* where it is activated by a ${ }^{252} \mathrm{Cf}$ neutron source. The activated solution then flows through detectors that measure delayed neutrons and delayed gamma rays. The delayed-neutron

*The irradiation chamber is in the international patent process. 


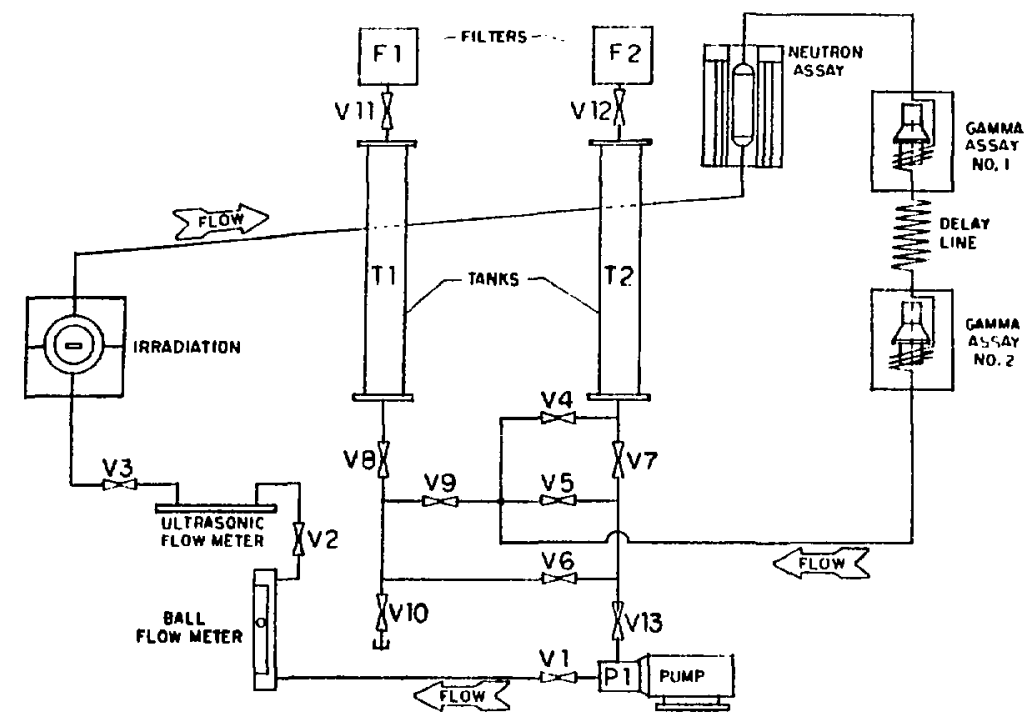

Fig. 29. Schematic diagram of the flowing fissile assay system.

signal is proportional to concentration. and the delayed gamma-ray signals, measured at two different distances from the irradiation chamber, can be used to determine volumetric flow rate and concentration.

The prototype test loop shown in Fig. 30 was completed during FY 1983. We used aluminum solutions to test the operation of the system and compared the calculated mass flow rates and material concentrations with experimental data. The measured results agreed with known values within the uncertainties of the measurements. We expect to begin tests in February 1984 using uranium nitrate solutions in different concentrations and enrichments.

This technique for measuring flowing streams may also have application in uranium solution mining, oil shale recovery, coal slurry transportation, and any flowing process that can be induced to give a nuclear signature.

8. Direct Fissile Assay of Highly Enriched UF $_{6}$ Cylinders Using Passive Neutron Coincidence Counting (J. E. Stewart and H. O. Menlove, Q-1). Measurement of ${ }^{235} \mathrm{U}$ mass in uranium hexafluoride is required for the accountability of HEU. The $\mathrm{UF}_{6}$ enriched to more than $5 \%{ }^{235} \mathrm{U}$ is stored in Model $5 \mathrm{~A}$ cylinders that are nominally $128 \mathrm{~mm}$ in diameter and $914 \mathrm{~mm}$ tall. Typical fill heights are 300 to $400 \mathrm{~mm}$. These containers, holding material of high economic and strategic value,

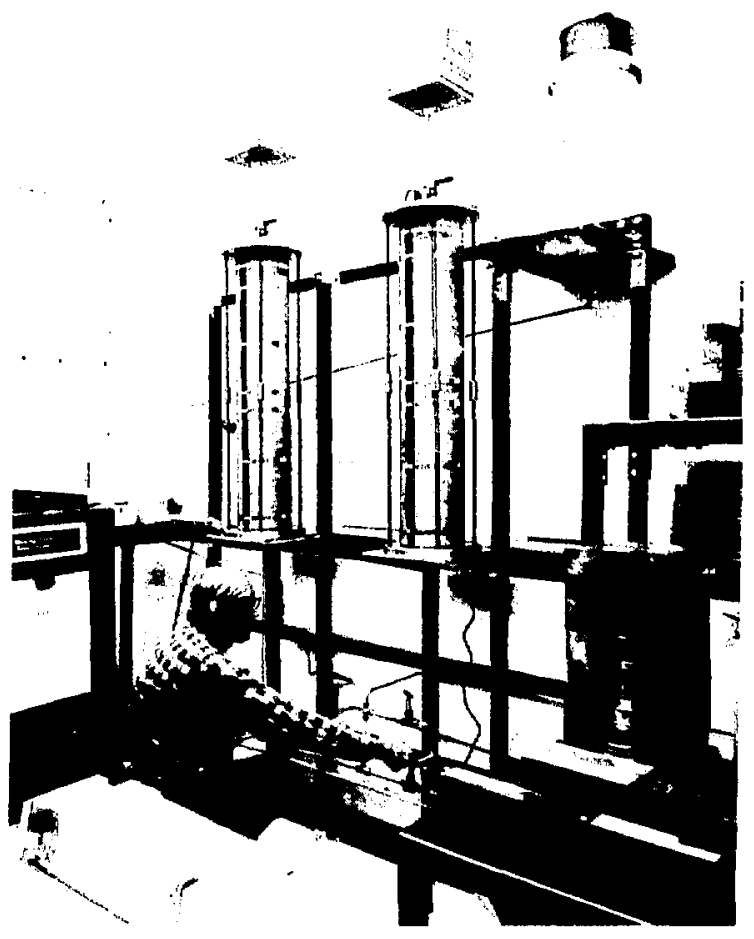

Fig. 30. The prototype flowing fissile assay system assembled in 1983 to test the new assay technique on actual nuclear material solutions. 
are used for the output of enrichment plants and for the input to fuel fabrication facilities.

Conventional nondestructive measurement of $5 \mathrm{~A}$ cylinders uses the 'enrichment-meter' principle. ${ }^{33}$ The 186-keV gamma rays from ${ }^{235} \mathrm{U}$ are counted using sodium iodide or germanium detectors. An ultrasonic measurement is made of the cylinder's wall thickness (6.4-mm Monel or steel) to correct for variations in gamma-ray attenuation. This technique has the disadvantage that only the enrichment of the $\mathrm{UF}_{6}$ is measured directly. Furthermore, only the surface layer (less than $11 \mathrm{~mm}$ ) of the $\mathrm{UF}_{6}$ is sampled; the bulk of the material in the interior is not verified. Furthermore, if the uranium has been irradiated in a reactor, it may contain levels of technetium that limit the precision of the gamma-ray enrichment measurement.

We have developed a new passive assay technique that directly measures the entire ${ }^{235} \mathrm{U}$ mass in a $5 \mathrm{~A}$ cylinder. We identified this technique, based on neutron self-interrogation and coincidence counting, after evaluating both active and passive applications of the Neutron Coincidence Collar, ${ }^{34}$ and tested it using the Active Well Coincidence Counter (AWCC $)^{35}$ in the passive mode, the Dual Range Coincidence Counter (DRCC), ${ }^{36}$ and the High-Level Neutron Coincidence Counter (HLNCC).$^{37}$ The first field test of the method was conducted in September 1983 at the Goodyear Atomic Corporation GDP near Piketon, Ohio, using a high-efficiency AWCC in the passive mode.

The new method owes its simplicity to the unique neutronic properties of highly enriched $\mathrm{UF}_{6}$ and the ability of shift-register coincidence circuitry to isolate time-correlated events from random events. Variations in cylinder-wall thickness or the presence of reactorreturn material do not significantly affect the measurement results. However, variations in fill height and $\mathrm{UF}_{6}$ density do affect the measurement, and we have developed a correction to minimize these effects. This correction is determined by changing the neutron reflectivity (albedo) of the sample cavity with a removable cadmium liner.

The technique uses the ratio of coincidences to totals as a measure of bulk fissile mass. The accuracy of the method is $6.8 \%$ ( $(\sigma)$ based on field measurements of 44 cylinders, 11 of which were only partially filled or contained reactor-return material. The cylinders contained $\mathrm{UF}_{6}$ with enrichments from $5.96 \%$ to $97.6 \%$. Count times were from 3 to 6 min depending on ${ }^{235} \mathrm{U}$ mass. Sample masses ranged from less than $1 \mathrm{~kg}$ to over $16 \mathrm{~kg}$ of ${ }^{235} \mathrm{U}$. Figure 31 shows field assay results and the calibration curve; Fig. 32, Goodyear personnel lowering a cylinder into the AWCC with a forklift.

Because the method relies primarily on fast-neutron self-interrogation, complete sampling of the $U F_{6}$ takes place. This feature alleviates inhomogeneity problems

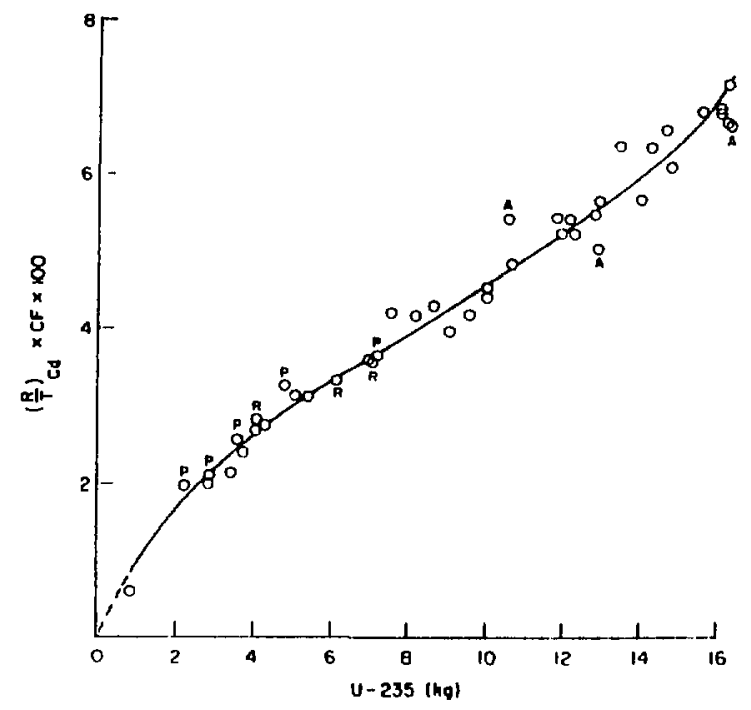

Fig. 31. The corrected, passive $(R / T)_{C d}$ signature vs ${ }^{235} U$ mass for field measurements of 44 Model 5A cylinders. The solid curve is an unweighted, least squares polynomial fit to the data.

and offers increased assurance of the presence of stated amounts of bulk fissile material.

The feasibility of this new technique for direct fissile mass verification of highly enriched $U_{6}$ has been successfully demonstrated in the laboratory and during the first field test. It is the first nondestructive assay technique for verifying the entire fissile mass in a $5 \mathrm{~A}$ cylinder. Further measurements are planned at Goodyear in the spring of 1984; another field test may take place during 1984 at a European fuel facility to demonstrate the technique to EURATOM and IAEA

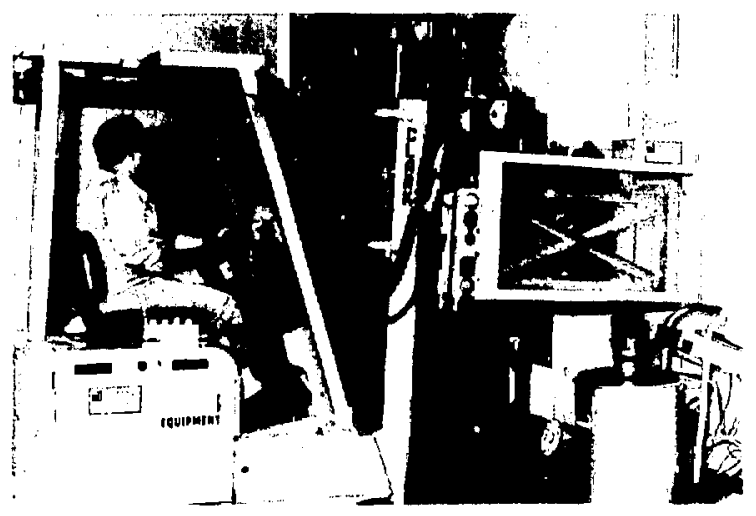

Fig. 32. Goodyear personnel lower a $5 \mathrm{~A} \mathrm{UF}_{6}$ cylinder into an $\mathrm{AWCC}$ during a field test of the new measurement technique. 
safeguards inspectors. The technique is under consideration to measure the input UF $_{6}$ cylinders at the new US Naval FMF.

9. Hybrid Assay System (S.-T. Hsue, and T. Marks, Q-1; S. F. Marsh, CHM-1). Plutonium scrap and waste recovery involves dissolution in nitric acid and subsequent separation by the ion-exchange process. The typical effluent solution generated in this process has a low plutonium concentration (less than $100 \mathrm{mg} / \mathrm{l}$ ) and a relatively high americium concentration (americium/ plutonium ratio of about 20). Because large quantities of this kind of waste solution are generated, timely assays of the plutonium content are important for process control and accountability.

The measurement of low plutonium concentrations requires more abundant signals than those produced by the passive gamma rays of plutonium. The $L \times$ rays induced during plutonium alpha decay are nearly 100 times more abundant than are plutonium gamma rays. Whereas plutonium decay gives rise to uranium $\mathrm{x}$ rays, the decay of americium and ${ }^{237} U$ gives rise to neptunium $x$ rays. The major limitation to using uranium $L x$ rays for the assay of plutonium in effluent solutions is interference from neptunium $x$ rays. For an americium/ plutonium weight ratio of 1 , the $N p L \beta_{1} / U L \beta_{1}$ peak area ratio is approximately 290 , which means the uranium $\mathrm{x}$ ray is impossible to measure.

We have demonstrated that a simple and rapid solvent extraction can effectively separate the plutonium from americium, so that the uranium $x$ rays can be measured with little interference. The extraction is based on the property that trioctylphosphine oxide forms complexes with plutonium that preferentially dissolve in the organic solvent, in this case, kerosene.

We have developed an instrument based on this hybrid chemical/NDA technique to assay the plutonium concentration in ion-exchange effluents. It is designed to assay both separated and nonseparated samples. This device (LOSAI), shown in Fig. 33, was installed in the Los Alamos Plutonium Facility more than a year ago for test and evaluation (T\&E). After successful completion of the T\&E, the LOSAI was used for accountability purposes. It can assay plutonium concentrations as low as $1 \mathrm{mg} / \mathrm{l}$ with an accuracy of about $15 \%$.

At the request of the SRP, we developed a second instrument based on similar measurement principles. The major change in this instrument is that a responsefunction technique is used to fit the complex uranium and neptunium $L \beta_{1}$ peaks. This technique enables the instrument to tolerate higher americium/plutonium ratio samples without the necessity of chemical separation. This instrument, named the Technique for Ef-

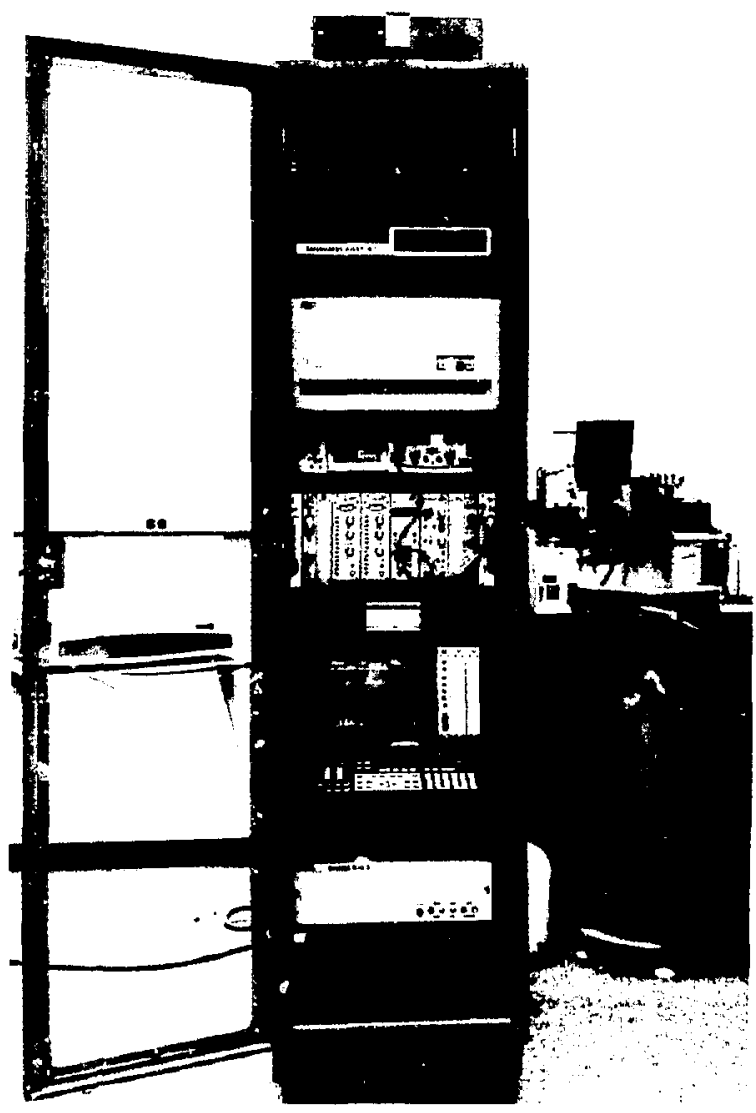

Fig. 33. The LOSAI before installation in the Los Alamos plutonium facility.

fluent Waste Assay (TEWA, Fig. 34), was shipped in September 1983 to SRL for test and evaluation. Later the TEWA will be transferred to the SRP to assay routine samples. Another similar instrument may be used in the SRP laboratory in place of alpha-counting plutonium solution samples. This is part of a plant upgrade effort to minimize the need to bring liquid samples out of the plant for chemical assay.

10. Uranium Monitor for Plutonium-Recovery IonExchange Columns (S. -T. Hsue, Q-1, and S. F. March, CHM-1). An ion-exchange-column process, based on preferential sorption of plutonium on anion-exchange resin in nitric acid medium, is often used to purify plutonium from other elements. The elution of uranium, one of these elements, is slow and requires large elution volumes. We have developed an on-line measurement system to monitor the uranium concentration in the column effluent to indicate when the 


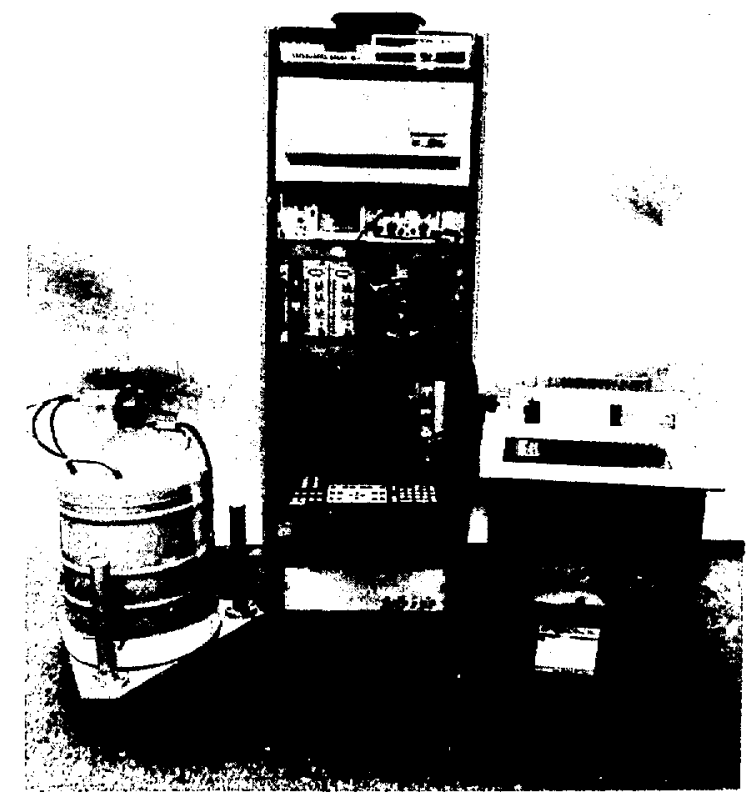

Fig. 34. The TEWA instrument before installation at SRP.

concentration reduces to a pre-established level so that elution can be stopped. This eliminates unnecessarily large elution volumes and the associated costs of processing them. The basis of the system is monitoring the 208-keV gamma ray of ${ }^{237} \mathrm{U}$, the 6.75 -day half-life daughter of ${ }^{241} \mathrm{Pu}$ that serves as an internal tracer for uranium.

A prototype of this monitor on a laboratory scale provided the means to effectively evaluate process variables of anion-exchange resin types and nitric acid concentration to achieve a more rapid, and hence, lower elution volume of uranium. A threefold reduction in elution volume was obtained by replacing the conventional Dowex-1 $\times 4$ resin with macioporous anionexchange resin and by reducing the nitric acid concentration from $8 \mathrm{M}$ to $4-5 \mathrm{M}$.

We have assembled and installed an instrument at the Los Alamos Plutonium Processing Facility for T\&E. The concept has been expanded to monitor plutonium and americium in addition to uranium in the effluent by measuring characteristic gamma rays from each element. Evaluation of the system for monitoring uranium has been delayed until processing of uranium-containing material resumes; however, testing of the system for monitoring plutonium and americium is in progress, and preliminary results are very satisfactory.

\section{B. Spent-Fuel Measurement}

1. Spent-Fuel Measurement System (G. E. Bosler, J. Halbig, S. F. Klosterbuer, and P. M. Rinard, Q-1). For several years we have been developing equipment and techniques to measure spent-fuel assemblies, with emphasis on equipment that an inspector can use easily in a spent-fuel pond. In this application, inspectors must verify that objects stored underwater are indeed spentfuel assemblies with the proper amount of nuclear material. The verification measurements are done underwater, and some movement is required to isolate an individual assembly from others in a storage rack.

The instrumentation (Fig. 35) includes an ION-1 portable gamma-ray and neutron electronics unit and a polyethylene "fork" detector. The other instrument shown in the figure is an Epson HX-20 computer that can be attached to the ION-1 to record and analyze data. The ION-1 is a self-contained, battery-operated microprocessor designed for use on a spent-fuel pond bridge. The software is designed to prompt the user and assist in making measurements.

The fork detector head contains two ion chambers and two fission chambers to measure the gamma-ray and neutron signals from a spent-fuel assembly. The

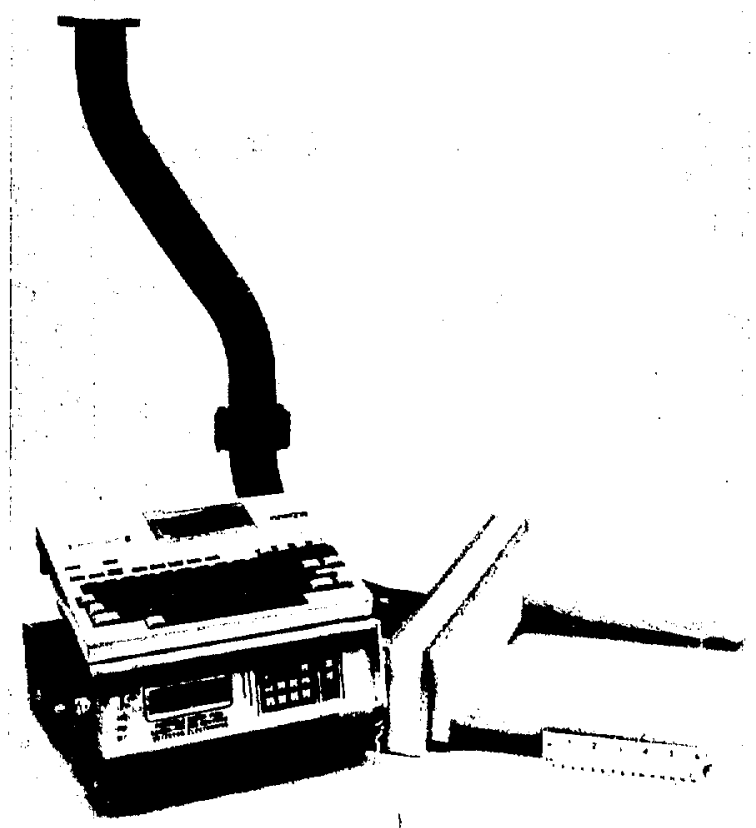

Fig. 35. Principal components of ION-1/FORK detector system for measuring spent-fuel assemblies. 
measurement is shown schematically in Fig. 36. Sections of pipe are attached to the storage-pend bridge and used to suspend the fork detector above the storage rack. The distance above the rack is determined by the background gamma radiation level. Measurements are made by positioning the fork over a fuel assembly, raising the assembly, and moving the fork arms around the assembly with the back of the fork in contact with the assembly.

A typical measurement requires 5 to $7 \mathrm{~min}$ at a single axial position (usually the midpoint). The time to achieve $1 \%$ relative counting statistics in the neutron channel is usually from 20 to $30 \mathrm{~s}$; simultaneous gamma measurements take $10 \mathrm{~s}$ or less. The remaining time is required to move the detector and the fuel.

Neutron and gamma-ray data from spent-fuel assemblies are used to verify the consistency of operatordeclared information, such as assembly exposure and cooling time. During 1983, the equipment was used in an actual facility inspection in the United States, at a

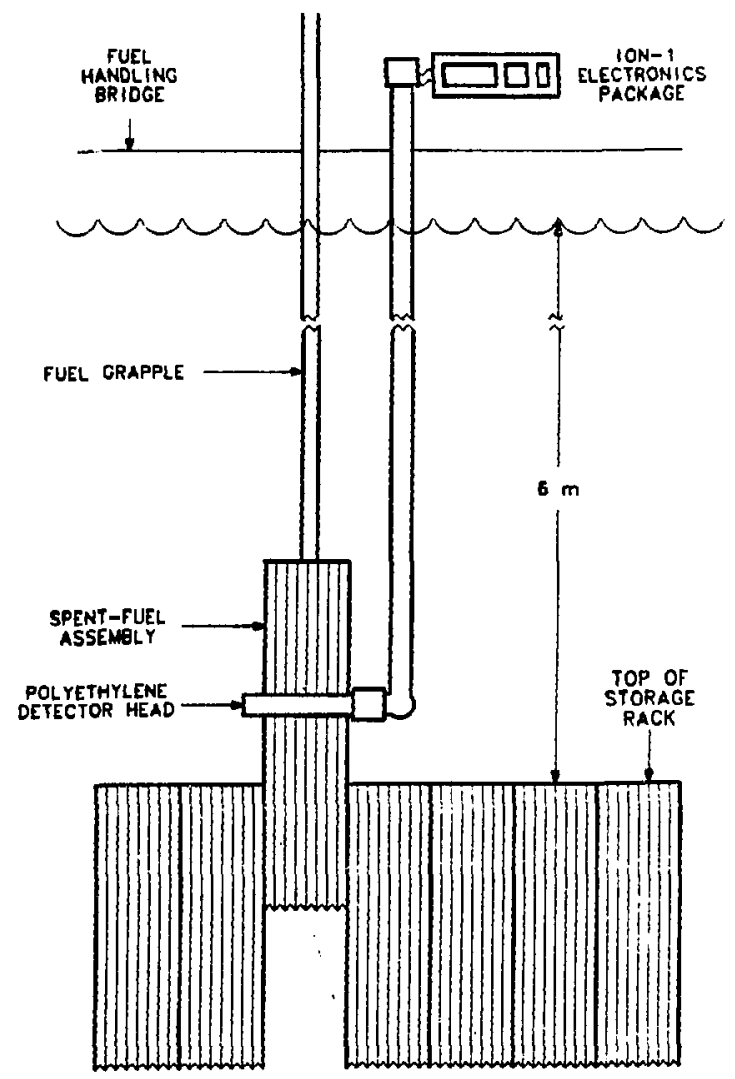

Fig. 36. Measurement configuration for using the ION-1/FORK detector system in a spent-fuel pond. data-gathering exercise in Finland, and at demonstrations in two facilities in Germany. In each case the operator-declared information has been verified. Through these exercises we have gained considerable experience in using the equipment and in evaluating the data.

The use of passive-neutron and gamma-ray measurement techniques in the IAEA system is dictated by the need for portable (transportable) equipment. One problem is that neither signal is a direct measure of the fissile content. Such information is obtained either through correlations between the measured neutron/gamma data and the amount of fissile material present or through more complicated active-neutron measurements. Both of these methods are being investigated for future applications.

In a correlation method, passive signals are used to determine the burnup of an assembly, which, in turn, is related to the fissile content. Correlations between burnup and fissile content are established through a combination of experimental and calculational techniques. The biggest problems with such a correlation method are establishing a data base and being able to make enough measurements to verify the method.

In an active-neutron measurement, the fissile content is determined by inducing fission in the assembly and measuring the resulting fission rate. The most common method for inducing fission is using an external source. A major problem is the size $\left(10^{7}\right.$ to $\left.10^{8} \mathrm{n} / \mathrm{s}\right)$ of the source needed to overcome the inherent neutron background in a spent-fuel assembly. Such a large source is difficult to transport and, therefore, is not considered practical for portable applications. Unless portable neutron generators with sufficiently high yields become available, active source techniques will be confined to stationary, in-plant installations.

An alternative to the use of a radioactive source is the self-interrogation method in which neutrons from the assembly are reflected back into the assembly and the resulting induced-fission rate is measured. This method obviously provides a direct measurement of the fissile content and does not require the transportation of large sources. We are planning to use the existing fork detector with a reflector to evaluate the effectiveness of the self-interrogation method.

While our main effort over the past few years has been aimed primarily at building portable equipment suitable for use by the IAEA, the techniques are applicable for other uses. An assay system that is permanently installed in a facility has previously been proposed. ${ }^{38,39}$ Such a system would be more precise than a portable system and could be calibrated to determine fissile content. If it were installed at a reprocessing plant, 
a system of this type could be used to resolve shipper/receiver differences, and at a reactor facility, the system would be used to assay spent-fuel assemblies and verify operator-declared values.

2. Cerenkov Viewing Device (CVD) (N. Nicholson, Q-2). With CVDs or improved CVDs (ICVDs), an IAEA inspector can monitor and measure the Cerenkov glow emitted from irradiated fuel assemblies stored underwater. However, some nations are reluctant to extinguish normal lighting in the spent-fuel assembly storage areas. We have conducted a proof-of-principle experiment that demonstrates the feasibility of using a CVD or an ICVD in a normally lit room to view the Cerenkov glow from spent fuel as though the room lights were turned off.

This approach requires that a set of strobe lights replace the normal lighting in the spent-fuel bay. The strobe lights are pulsed on and off ai a rate fast enough to avoid the flickering strobe effect, and a mechanical chopper in the optical system of the CVD or ICVD is synchronized with the bank of strobe lights. This chopper prevents the strobe light from entering the Cerenkov instrument, but opens when the strobe light is off. Thus, the instrument can view the Cerenkov glow between light pulses.

In the proof-of-principle experiment, a mechanical chopper was attached to an ICVD that was viewing a light source equivalent in brightness to a spent-fuel assembly with low burnup and long cooling time. A strobe light was used to illuminate the room, but the weak light source was still easily visible, and the photometric reading on the ICVD was unchanged. A schematic of the experimental setup is shown in Fig. 37. Synchronization between strobe light and mechanical chopper was achieved by using a single-pulse generator to drive both systems. The light source used to simulate a spent-fuel assembly was an American Optical microscope light set on the number five brightness position (most dim) with an Incanol filter, ND-8, in front of the

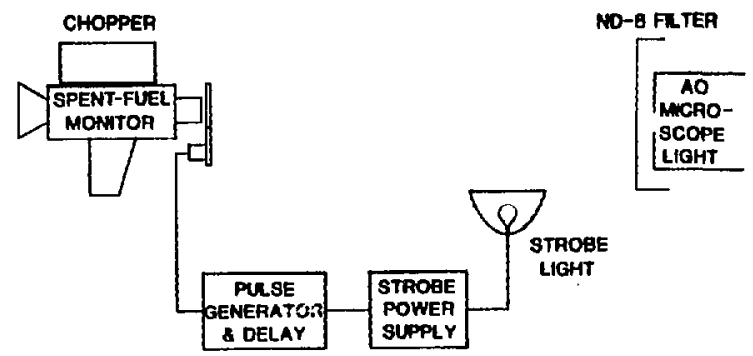

Fig. 37. Schematic of experimental setup. microscope light. By adjusting the timing properly, the mechanical chopper allowed the spent-fuel monitor to view the very weak light emitted by the microscope light attenuated by the ND-8 filter during the time when the strobe light was off. The light level produced by the strobe light was sufficient to illuminate a modest-sized laboratory and at a rate at which no noticeable flickering was observed.

\section{Containment and Surveillance Technology Develop- ment}

1. SNM Monitor R\&D (P. E. Fehlau, K. L. Coop, K. V. Nixon, S. W. France, J. Martinez, and C. Garcia, Q-2). This project includes evolutionary work on SNM radiation monitors ${ }^{12}$ and radiation-monitoring techniques as well as basic technical assistance for users of SNM monitoring equipment. During 1983 we developed the roadbed SNM monitor into a highly sensitive vehicle monitor for enriched uranium. The vehicle monitor controller uses sequential probability ratio decision logic, a newly investigated monitoring technique; other investigations evaluated new equipment and detectors. Finally, we observed SNM monitoring equipment in use at four DOE facilities and proposed training and maintenance aids to ease calibrating and servicing the monitors.

a. SNM Vehicle Monitor. Our evaluation of vehicle SNM monitors revealed that the addition of overhead detectors to a roadbed SINM vehicle monitor would greatly increase its sensitivity for detecting highly enriched uranium. In addition to constructing a space frame to support the overhead detectors (Fig. 38), we enhanced the monitor's performance by adding a personnel SNM monitor for pedestrians and vehicle occupants, moving the electronics to a locked cabinet and providing remote information and control modules for the guards, and changing the monitor's decision logic to a sequential probability ratio technique that permits faster decisions in most cases. The more rapid decision capability of the control logic reduces the average amount of time that a vehicle must remain stationary in the monitor, increases exit-station throughput, and makes the monitoring delay more acceptable to departing personnel.

Construction of the monitor is complete, and the new equipment is being installed. In addition, new scintillators will replace the original neutron and small $\mathrm{NaI}(\mathrm{Tl})$ gamma-ray detectors in the roadbed. These new detectors are large plastic scintillators that will make best use of the available space for detection of enriched 


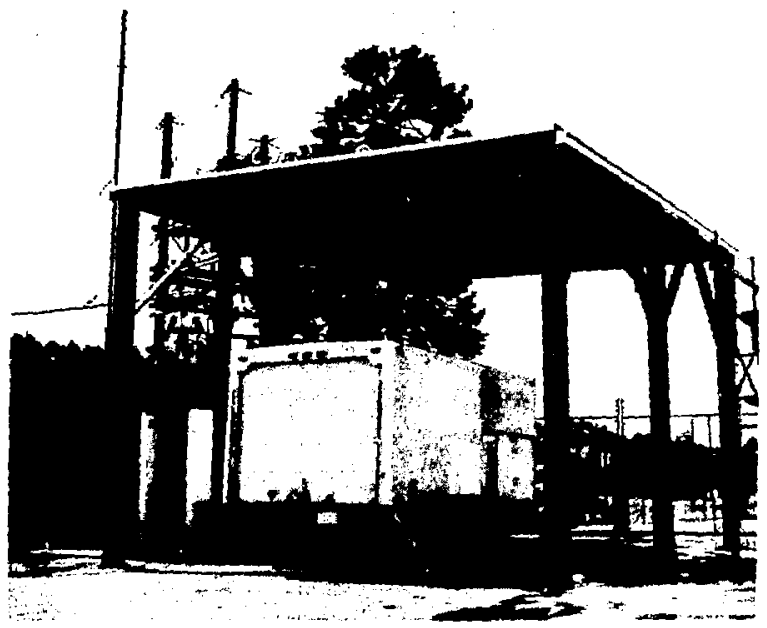

Fig. 38. A space frame over the roadbed monitor detectors will support overhead detectors. The additional detectors along with improved detection logic and new detectors will greatly improve the monitors's ability to detect highly enriched uranium.

uranium. New detector cabinets for the scintillators should reduce maintenance frequency and will make necessary repairs easier.

b. SNM Monitors at DOE Facilities. We observed SNM monitors, most of them personnel monitors, in everyday use at Los Alamos, Mound Laboratory, the SRP, and the Rocky Flats Plant. We found no evidence of any basic design flaw in the commercial equipment, but there is a shortage of good documentation for the personnel who calibrate and service the equipment. The original service manuals are often lost or unavailable when maintenance takes place. Calibration charts should be attached to each monitor, and sufficient training and service documentation should be provided to ensure that the SNM monitors are operated to meet the needs of DOE security.

In addition, general information on the applications and implementation of SNM monitors is needed. We are considering a workshop on SNM monitoring for security managers perhaps as part of an INMM annual meeting. Documentation for the course would provide up-to-date information for those who are unable to attend.

Our first effort at providing up-to-date documentation is nearly complete. This is a user's manual for the hand-held SNM monitor and was developed with the assistance of the Los Alamos Protective Force (Fig. 39). It is being produced in a convenient size for use at guard stations where hand monitoring is required and gives a

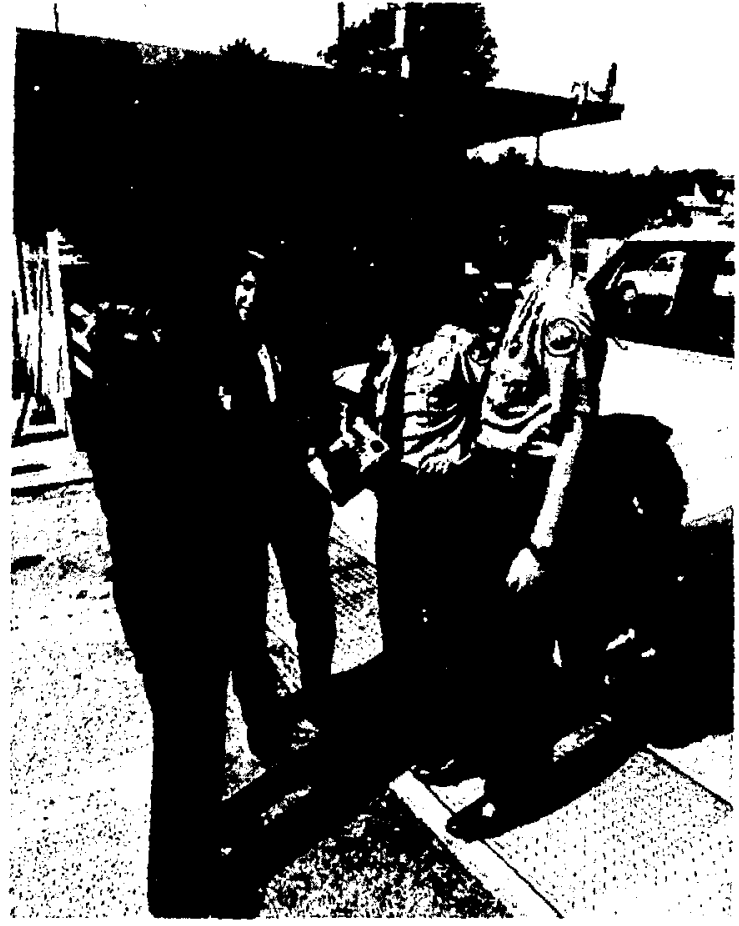

Fig. 39. A users manual for hand-held SNM monitors was developed with the assistance of the protective force at Los Alamos. The manual has been published as a Los Alamos brochure.

very general introduction to SNM monitoring and specific instruction on hand-monitoring techniques for personnel, vehicles, and packages. It has been circulated to security managers at many DOE facilities and to hand-held monitor manufacturers for review. Many useful suggestions were received and are being incorporated into the final edition of the manual.

Another step toward better documentation began as part of a program for the NRC to document nondestructive assay techniques. A chapter on SNM monitors written for the NRC report will serve as a starting point for a stand-alone report on present-day SNM monitoring techniques and equipment.

c. New Equipment and Techniques. We evaluated a new SNM hand-held monitor and investigated newly developed small photomultipliers as a way to make hand-held monitors smaller and lighter. The commercial hand-held monitor is a TSA Systems, Inc., model HHD-440, which is shown in Fig. 40 with a commercial hand-held monitor and a prototype lightweight SNM hand-held monitor, the programmable rate 


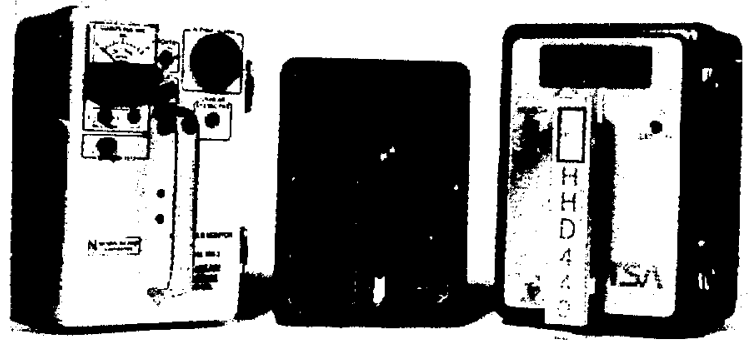

Fig. 40. Hand-held SNM Monitors are available in a variety of sizes. The HHD-440 at the right is somewhat heavier and larger than the commercial monitor at the left. The PRM at the center is the smallest and lightest monitor of this type.

meter (PRM), developed at Los Alamos. The TSA monitor met DOE specifications for performance but was somewhat heavier than other commercial monitors. The HHD-440 detection logic is the first application, in a hand-held monitor, of the sliding-interval technique developed at Los Alamos for personnel monitors. ${ }^{13}$ The sliding-interval technique enhances the HHD- 440 sensitivity enough that it could use a much smaller, lighter detector and still meet DOE specifications. Another new Los Alamos technique is applied commercially for the first time in this monitor. The variance analysis technique ${ }^{40}$ is a calibration and troubleshooting aid developed for the SNM vehicle portal. TSA Systems, which adopted the technique for the HHD-440 to aid detector calibration, also uses the technique in its personnel and vehicle monitors.

We investigated the effect of detector size on detection sensitivity in hand-held monitors. We considered alternative sizes of $\mathrm{NaI}(\mathrm{Tl})$ scintillators and also examined the HHD-440 plastic detector and two new detector materials, $\mathrm{HgI}_{2}$ and bismuth germanate. Our results $^{41}$ indicated that performance equal to existing monitors could be obtained with small $2.54-\mathrm{cm}$-diam photomultipliers used with similar-sized $\mathrm{NaI}(\mathrm{TI})$ scintillators that are much lighter than those found in existing commercial monitors. We incorporated such a detector in a prototype lightweight monitor, the PRM (Fig. 41), that also incorporates a lightweight power supply (Fig. 42). We also have purchased a demountable version of the HHD-440 so that we can remove its detector and evaluate alternative lightweight detectors together with the more-effective sliding-interval detection logic.

A new method for detecting transient increases in radiation intensity, sequential probability ratio testing (SPRT), was introduced in the fall 1982 safeguards progress report. ${ }^{21}$ Since then, we have investigated the

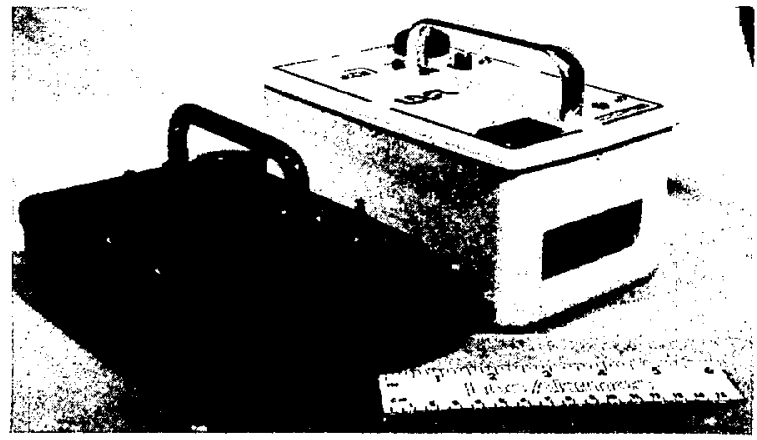

Fig. 41. The difference in size between the new lightweight PRM and a commercial hand-held SNM monitor is evident in this view. The PRM is also about $45 \%$ lighter in weight than the commercial unit.

performance of the technique and incorporated it into hardware for controlling SNM monitors. The advantage of sequential testing is the early decision made possible by repeated tests during the monitoring period. Figure 43 shows the average number of monitoring tests required to make a decision for different signal intensities. For both background intensity and large anomalous signals, the decision time is very short. At its worst (near the alarm point), the average decision time is equal to that of other methods. Hence, this decision logic method reduces overall monitoring times (from $3 \mathrm{~s}$ to $0.6 \mathrm{~s}$ for an SNM monitoring booth ${ }^{42}$ and from $50 \mathrm{~s}$ to about $12 \mathrm{~s}$ for a vehicle monitor). ${ }^{13}$ For monitoring moving personnel or vehicles in portal SNM monitors, the rapid decision capability permits the monitor to detect transient intensity increases rapidly. Because the transient need not be present for any preselected period of time, vehicle or pedestrian speed has less effect on

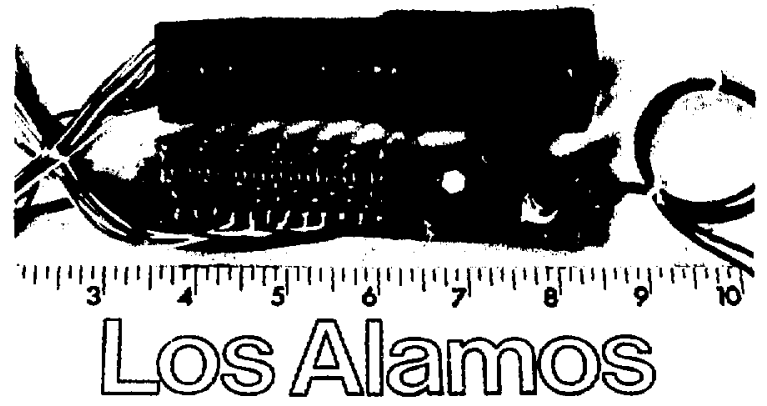

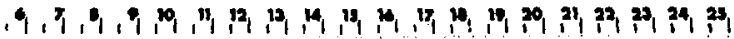

Fig. 42. A small high-voltage power supply provides individual dynode voltages for the PRN's small NaI(Tl) scintillation detector. This method of achieving dynode voltages minimizes the power requirement and allows the instrument to operate with lightweight batteries as long as $45 \mathrm{~h}$ before recharging. 


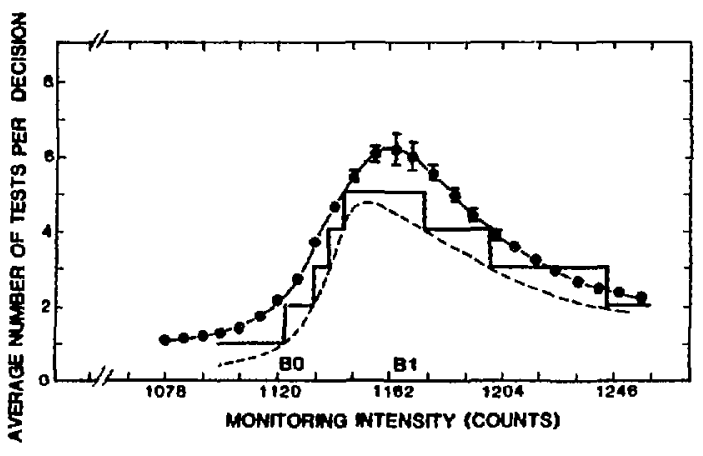

Fig. 43. The sequential technique quickly determines intensities near background intensity (BO) or very large intensities. At its worst, near the alarm threshold ( $\mathrm{B} 1$ ), the technique requires as long on the average as other techniques. The curves represent observations (solid line) and predicted behavior.

detection sensitivity than in other monitoring methods. A vehicle monitor controller incorporating the sequential method (Fig. 44) is part of the SNM vehicle monitor for enriched uranium described above. This controller has four independent channels, each having its own sequential decision logic, for monitoring different areas of each vehicle.

2. Reactor Power Monitor (RPM) (R. D. Hastings, Q-2). The RPM is an example of instrumentation successfully applied to extended containment and surveillance. The prototype instrument provided to the IAEA was recently field tested for 94 days at a power reactor site in the far east. Although early attempts by field personnel to read out data were unsuccessful, when the instrument was returned to Los Alamos, we found that the readout unit (a Texas Instrument terminal) had been damaged. The RPM had performed satisfactorily, and we were able to read out the data. We do not yet have the facility operator's records for comparison.

\section{Chemical and Isotopic Analysis}

1. Development of Dissolution Technology (S. F. Marsh, CHM-1). Many nuclear-fuel-cycle materials are refractory and difficult to dissolve, whereas highly reliable chemical analyses require complete dissolution of solids. As part of an investigation of new dissolution systems, we have evaluated two fusion media, molten lithium metaborate $\left(\mathrm{LiBO}_{2}\right)$ and molten magnesium

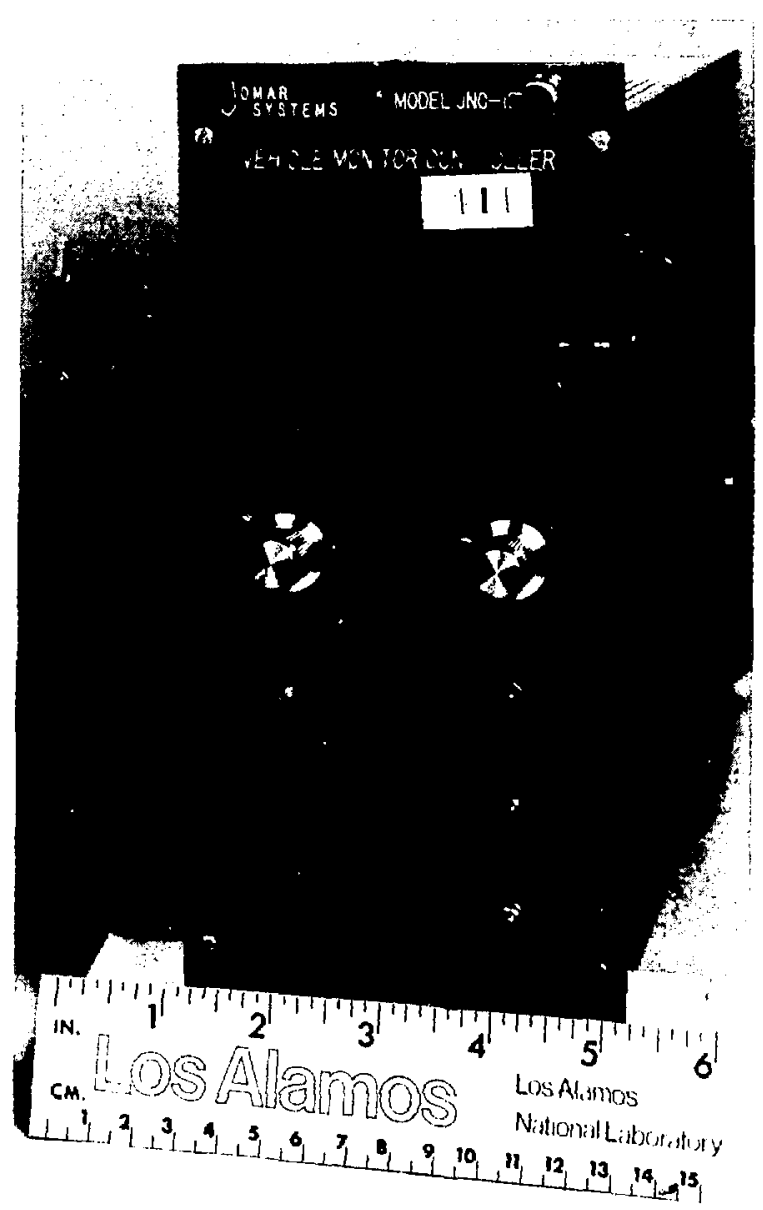

Fig. 44. The sequential testing technique has been incorporated in a control module for the SNM vehicle monitor. The module has four separate channels that are independently monitored. The channels correspond to discrete groups of detectors in the monitor and when each of them is separatcly controlled, the monitor's sensitivity increases.

metal, as dissolvents for highly refractory, $1600^{\circ} \mathrm{C}$ calcined plutonium oxide.

Of various container materials tested, only graphite resisted attack with both fusion media. We used a tube furnace flushed continuously with argon and vigorous oxidation of the magnesium metal to prevent oxidation of the graphite. We achieved limited success for test material consisting of pieces of sintered plutonium oxide. At the furnace operating temperature of $950^{\circ} \mathrm{C}$, the magnesium metal volatilized before significant reaction occurred, so we are considering a closed container for 
this reaction. Because the fusion with $\mathrm{LiBO}_{2}$ only partially decomposed the plutonium oxide, we are investigating factors, such as increased temperature, increased reaction time, and smaller pieces of sample, which we expect to increase the decomposition rate.

2. Automated Complexometric Titrator for Uranium (R. M. Hollen and D. D. Jackson, CHM-1). Construction of this instrument, which determines microgram amounts of uranium, has been completed. It uses a highly selective method in which the U(VI)-arsenazo complex is titrated with pyridine-2, 6-dicarboxylic acid (PDA) to a colorimetric endpoint. ${ }^{43}$

The instrument, whose mechanical components were described in a previous progress report, is controlled by a Hewlett-Packard HP-85 programmable calculator, and the measurement is completely automated including reagent addition, titration, cleaning of sample cells and other components that contact the samples, data reduction, and output. Output is a paper tape printout giving sample identification from the 36-position turntable and titrant volume, which is directly proportional to uranium quantity. A real-time plot of absorbence versus titrant volume provides diagnostic information. An interface monitors the interlocks that provide termination of instrument operation in case of possible damage or termination of the present analysis of a sample followed by resumption of operation in case of a malfunction affecting analysis reliability. The concentration of the hexamethylenetetramine buffer was increased relative to the original method ${ }^{43}$ so that up to 16 milliequivalents of acid, equivalent to concentrated nitric acid in the $1-\mathrm{m}$ \& sample volume, can be tolerated. A PDA concentration of $0.002 \mathrm{M}$ and incremental additions of $0.02 \mathrm{~m} \ell$ were selected to give the best combination of measurement precision and short analysis times. The titration endpoint is calculated as the intersect of linear, least-squares fitted lines before and after the inflection point in the curve of absorbence versus titrant volume. The inflection point in the titration curve is located by monitoring the rate of change of absorbance. When the difference between measured absorbances is less than a preselected value for three consecutive times, the titration is stopped, and the endpoint is calculated. The instrument is calibrated by processing aliquots of uranium reference solution.

Uranium amounts of 20 to $300 \mu \mathrm{g}$ are determined at a rate of about one analysis each 5 minutes. If the quantity of uranium is outside the range of the instrument, a message is printed and analysis of that sample is terminated. Measurement precision is 1 to $2 \%$ RSD for
50 to $300 \mu \mathrm{g}$ of uranium. At the practical lower measurement limit of $20 \mu \mathrm{g}$ of uranium, the precision is $5 \%$ RSD.

We are completing evaluation of the instrument by investigating its tolerance for metal and nonmetal ions present in nuclear fuel-cycle materials.

\section{Controlled-Potential Coulometric Determination} of Uranium (N. M. Saponara and D. D. Jackson, CHM-1). We have developed a method for the controlled-potential coulometric determination of uranium at a solid plutonium electrode that is adaptable to the Los Alamos automated controlled-potentialcoulometric analyzer used for determining plutonium. The method features low milligram sensitivity, precision of $0.1 \%$ RSD at the 5-mg uranium level, high specificity, and long-term electrode stability.

Because $\mathrm{H}^{+}$reduces to $\mathrm{H}_{2}$ at the low potential required to reduce $U(V I)$ to a lower valence, none of the many systems previously investigated for directly determining uranium at a solid electrode was successful. The present method uses chemical reactions in conjunction with a platinum-mesh working electrode. The basis of the method is a chemical reduction of U(VI) to U(IV) with $\mathrm{Cr}(\mathrm{II})$ and oxidation of the U(IV) to U(VI) with electrogenerated $\mathrm{Fe}(\mathrm{III})$. The electrolyte is $0.5 \mathrm{M}$ sulfuric acid.

Iron is necessary for the oxidation of U(IV). For ironto-uranium ratios of less than 1, electrolysis times are long. At molar ratios of $1: 1$ or greater, oxidation times for $5 \mathrm{mg}$ of uranium are less than 20 minutes.

The results of a detailed investigation of the effect of 45 metal cations are presented in Table IX. Interference is defined as a change significant at the $\mathbf{9 5 \%}$ confidence level relative to uranium alone. The level of uranium

\begin{tabular}{cc}
\hline \hline TABLE IX. Tolerance Level of Metal Cations" \\
\hline $\begin{array}{c}\text { Mole Ratio } \\
\text { With Respect to } \mathrm{U}\end{array}$ & \multicolumn{1}{c}{ Cations } \\
\hline \multirow{2}{*}{$1: 1$} & $\mathrm{Al}, \mathrm{As}, \mathrm{B}, \mathrm{Be}, \mathrm{Ca}, \mathrm{Cd}, \mathrm{Ce}, \mathrm{Cr}$, \\
& $\mathrm{Co}, \mathrm{Fe}, \mathrm{Ga}, \mathrm{Hf}, \mathrm{In}, \mathrm{La}, \mathrm{Mn}, \mathrm{Na}$, \\
& $\mathrm{Nb}, \mathrm{Ni}, \mathrm{Os}, \mathrm{Pb}, \mathrm{Pu}, \mathrm{Th}, \mathrm{Tl}, \mathrm{Zn}$, \\
& $\mathrm{Zr}$ \\
$1: 10$ & $\mathrm{Sb}, \mathrm{Se}, \mathrm{Sn}, \mathrm{Rn}, \mathrm{Ta}$ \\
$1: 100$ & $\mathrm{Cu}, \mathrm{Hg}, \mathrm{Pd}, \mathrm{Pt}, \mathrm{Ru}, \mathrm{W}, \mathrm{V}$ \\
\hline
\end{tabular}

'Au, Bi, Ir, Mo, Re, Te interfered at 1:100. 
used for each test was $0.02 \mathrm{mmol}(5 \mathrm{mg})$. The initial molar ratio tested was $1: 1$. If a result was significantly different, lower ratios were tested. Although not all elements in the alkali and alkaline earth groups were tested, the results of representative members are considered to apply to all. Most elements normally found in nuclear fuel-cycle materials do not interfere. The elements osmium, selenium, and ruthenium did not interfere in the uranium determination but impaired electrode response so that sufficiently low current could not be attained in subsequent analyses.

To ensure applicability of the method for determining uranium in uranium-plutonium mixtures, we examined the tolerance for plutonium in greater detail. There were no significant differences in results for five measurements of equal ratios of uranium and plutonium relative to five measurements of uranium alone.

Nitrate, a very common anion, is tolerated at a molar ratio of 10:1, but higher molar ratios cause a positive bias. Nitrate and many other potentially-interfering, volatile anions are conveniently removed by fuming with sulfuric acid. This was demonstrated for $20 \mathrm{com}$ monly occurring anions initially present at a 100:1 molar ratio.

Same-day measurement precision was less than $0.1 \%$ RSD at the 5-mg uranium level. The RSD for 32 analyses measured over a 2-week period was $0.17 \%$. Even during investigation of diverse ion effects covering a 3week period, the precision of uranium-standard determinations remained less than $0.2 \%$ RSD.

\section{Automated Laser-Excitation Fluorescence} Uranium Analyzer (R. M. Hollen and D. D. Jackson, CHM-1). We are developing an automated analyzer for the determination, based on uranium fluorescence, of low microgram levels of uranium in solution. This technique is used manually at many laboratories with commercial equipment.

We have purchased a fluorescence spectrometer and modified the sample chamber to provide sample containment and accurate positioning of the cells and are investigating chemical and instrumental conditions for uranium fluorescence in solution. Excitation wavelengths over the range of approximately 280 to $400 \mathrm{~nm}$ produced uranium fluorescence in most systems, with the greatest fluorescence at the shorter wavelengths. Below $280 \mathrm{~nm}$, the background signal increases rapidly so this value was established as the minimum useful wavelength. The fluorescence of uranium in solution is very sensitive to quenching by various ions, $\mathrm{pH}$, temperature, and the uranium species in solution. We are investigating these factors as well as techniques based on the long lifetime of the uranium phosphorescence.

5. Laser-Abetted Mass Spectrometer for Measuring Uranium and Plutonium Isotopic Distributions (J. W. Early, CHM-1). The objective of this activity is the development of a mass spectrometer that will determine accurately the isotopic distributions of uranium and plutonium without need for prior chemical separations of these elements from the many other elements present in nuclear fuel-cycle materials. This should result in a considerable savings of time and cost. We are investigating two techniques to eliminate masses of impurity elements that are isobaric with uranium and plutonium isotopes. The first involves selective laser photoionization of the uranium and plutonium in the source of the mass spectrometer. The second technique, termed ionselective laser acceleration, uses resonance radiation pressure to accelerate selectively and thereby defocus impurity element masses that are isobaric with uranium and plutonium isotopes.

Proof-of-principle calculations indicate the ion-selective laser acceleration method is technically feasible, and we have designed and are fabricating an apparatus to evaluate it. We will measure first the deflection of a beam of neutral barium atoms, which have simple and well-documented spectra and then a beam of neutral uranium atoms. If these experiments are successful, we will create the best operating conditions and interface the ion-selective laser acceleration unit with a magneticsector mass spectrometer for final evaluation of the technique.

6. Plutonium-Isotope Half-Life Measurements (R. M. Abernathy, S. F. Marsh, G. M. Matlack, and J. E. Rein, CHM-1; F. J. Steinkunger, INC-3; and R. J. Beckman, S-1). The goal of this DOE-sponsored interlaboratory program is the establishment of accurate plutoniumisotope half-life values. Values for ${ }^{239} \mathrm{Pu}$ and ${ }^{241} \mathrm{Pu}$ have been published, and publication of three Los Alamos papers on the measurement of the ${ }^{240} \mathrm{Pu}$ half-life is anticipated in the March 1984 issue of the International Journal of Applied Radiation and Isotopes. These papers describe our preparation and the interlaboratory characterizations of a batch of ${ }^{240} \mathrm{Pu}$ oxide used by the participating laboratories and our ${ }^{240} \mathrm{Pu}$ half-life measurements by alpha-particle counting and by isotope-dilution mass-spectrometric measurement of grown-in daughter ${ }^{236} \mathrm{U}$. The measured half-life is 6574 years with $95 \%$ confidence limits of \pm 12.8 years. 


\section{E. Standards Development}

1. Correction Factors for Sample Self-Attenuation in Gamma-Ray Assay (J. L. Parker, Q-1). Passive gammaray assay techniques, based on the detection of gamma rays emitted from nuclear materials with sodium iodide scintillation or germanium solid-state detectors, play an important role in the safeguards systems used to protect special nuclear materials. The most difficult part of these procedures usually is the determination of the fraction of the emitted gamma rays that escape unscattered from the sample. Knowledge of this fraction, often called the self-attenuation correction, is required to obtain accurate assays. Because the fraction can be as low as $20 \%$ for large packages (several liters or more) containing significant quantities of nuclear material (hundreds of grams or more), large assay errors are possible if the fraction is not known accurately. A related requirement for accurate gamma-ray assays is the possession and proper use of calibration reference materials. Considerable effort has been devoted to understand what constitutes an appropriate calibration standard for gamma-ray assays as well as to develop calibration procedures that require a minimum number of standards.

During 1983, we prepared a report entitled "The Use of Calibration Standards and the Correction for Sample Self-Attenuation in Gamma-Ray Nondestructive Assay" (Los Alamos National Laboratory report LA-10045). This report describes in detail much of what has been learned about these important gamma-ray assay subjects during the last 13 years. In the late sixties and early seventies, the approach to the problems of calibration and self-attenuation correction was to use "representative" standards, which were required to be similar to the assay samples in size, shape, and chemical composition. The count rates from a series of such standards were compared directly with those from the unknown samples without any explicit correction for self-attenuation. This procedure has serious difficulties because it often is impossible to know whether the standards adequately resemble the unknowns. Also the number of calibration standards can easily become large if there are many categories of material to be measured.

One goal of LA-10045 is to describe procedures to determine self-attenuation corrections using calibration standards that need not be chemically or physically similar to the samples being assayed. These procedures permit accurate calibration of gamma-ray assay systems using a relatively small set of standards. Thus calibrated, a system can assay accurately items covering a wide range of size, shape, chemical composition, and nuclear-material mass. These procedures save time, materials, effort, and expense.

The report documents much of the valuable experience and knowledge that Los Alamos has gained over the years through the study of gamma-ray assay techniques and makes the information available to the general safeguards community.

2. Consensus Standards Development (R. G. Gutmacher and A. S. Goldman, Q-4; S. -T. Hsue, T. E. Sampson, and T. D. Reilly, Q-1; and D. B. Smith, QDO/SG). Los Alamos safeguards personnel have participated in the standards writing activities of American Society for Testing and Materials (ASTM) Committee C-26 on the Nuclear Fuel Cycle. A new Task Group on Plutonium Spectroscopy, chaired by a Los Alamos representative, has completed work on a Standard Test Method for the Determination of Plutonium Isotopic Composition by Gamma-Ray Spectroscopy. The method, which has been submitted for committee ballot, applies to the determination of isotopic abundances in plutonium oxide, mixed oxide, and plutonium metal.

Future activities of the task group will include NDA methods for the determination of plutonium in solution and methods for the determination of total plutonium and uranium in scrap and waste. The task group currently has members from Battelle Pacific Northwest Laboratory, Ames Laboratory of Iowa State University, LLNL, Los Alamos, Mound Laboratory, NBL, NRC, ORNL, Rockwell Hanford Operations, Rockwell Rocky Flats Plant, SRL, and Westinghouse Hanford Company.

A Los Alamos safeguards representative actively participated in the revision of American National Standards Institute (ANSI) standard ANSI N15.19, "Volume Calibration Techniques for Nuclear Materials Control." This standard deals with volume calibration of liquid-holding tanks that are equipped with systems for measuring liquid content. Participation has been on Sections 7 and 8 dealing with statistical procedures for analyzing calibration data, which include methods for developing calibration models and discussion of how these models can be put to practicial use. Los Alamos also has responsibility for an appendix that applies these models to real data obtained from Savannah River.

During the year, ANSI published the standard "Guide to Preparing Calibration Material for Nondestructive Assay Systems that Count Passive Gamma Rays," ANSI N15.35-1983. This standard was developed by a standards writing group of the INMM. A Los Alamos staff member helped prepare this standard 
and continues to participate in efforts to develop other standards on the preparation of calibration reference materials.

Proposed ANSI standard ANSI N15.36, "Nondestructive Assay Measurement Control and Assurance," was approved for publication. This standard was prepared by writing group INMM 9.4, which is chaired by a Los Alamos staff member, and recommends procedures for establishing and operating a measurement control program to monitor and maintain the quality of NDA measurements.

3. Plutonium Metal Reference Material (J. E. Rein and Staff, CHM-1). We have completed packaging of the Los Alamos-produced batch of electrorefined plutonium metal as the seventh issue of this National Bureau of Standards (NBS)-NBL primary reference material. A plan was established, with N. Trahey of NBL, for its extensive chemical and isotopic characterization by NBL, ORNL, and Los Alamos. The NBL-requested completion date for the characterizations is April 1984 to provide time for statistical analysis and preparation of the certificate by the end of FY 1984. The Los Alamos characterization is complete.

4. Reference Materials for Calibration at NDA Instruments (Staff, CHM-1). Reference materials prepared for testing and calibrating NDA systems are summarized in Table $X$.

\section{MATERIALS CONTROL AND ACCOUNTING (MC\&A) TECHNOLOGY AND APPLICATIONS DEVELOPMENT}

\section{A. Design and Evaluation Methods for an Integrated Safeguards System (J. T. Markin, C. A. Coulter, R. G. Gutmacher, and W. J. Whitty, Q-4).}

A nuclear safeguards system is a collection of devices, procedures, and physical and personnel structures intended to protect nuclear materials from misuse. The NRC licensees and those DOE facilities or contractors that possess, use, or ship nuclear materials are required to implement appropriate safeguards systems for these materials. Most safeguards systems contain many elements, such as intrusion detectors, barriers to unauthorized entry, guards or security inspectors, "twoman" and other procedural rules, and elaborate materials accounting systems. The interactions of these elements with facility operations and with each other

TABLE X. NDA Calibration Materials

\begin{tabular}{cc}
\hline NDA System & Reference Material \\
\hline
\end{tabular}

Gamma Solution

Assay System containing

0.1 to $100 \mathrm{~g} \mathrm{U} / \mathrm{\ell}$

\section{Gamma and K-Edge}

Measurements

LIII-Edge Densitometer at ALNS Facility

Solution Assay Instrument

K-Edge Densitometer

Low-Solution Assay Instrument

L III-Edge Densitometer

\section{SAI Gamma Counter} the system often conflicting with goals for other parts. Systematic methods to design and evaluate safeguards systems to assure that they provide adequate protection for nuclear materials at minimum total cost have not been available.

We have developed general procedures for the design and evaluation of safeguards systems at either new or 
existing facilities, and have described these methods in a draft report submitted to DOE/OSS for comment. The design/evaluation process contains four phases:

(I) problem definition,

(2) system synthesis,

(3) system analysis/optimization, and

(4) decision making.

The relationships among these phases are shown in Fig. 45 , where it is indicated that iteration usually is required between the system analysis/synthesis and system evaluation/optimization stages to obtain a satisfactory final design.

In the problem definition phase, the specific performance goals of the safeguards system must be stated in detail, the design constraints (such as regulatory and facility requirements) determined, and a list of available safeguards resources (such as funding for the system and relevant technology) compiled. In addition, the nature of the possible threats to the facility must be determined by delineating the characteristics of adversaries who might threaten misuse of nuclear materials at the facility and by using specific facility characteristics in conjunction with a set of generic adversary actions to construct a list of possible adversary actions at the facility that would form the elements of all scenarios directed at misusing nuclear materials.

The synthesis phase begins with a hierarchical or "top-down" analysis stage in which the performance goals for the safeguards system are divided successively into lower-level subgoals until a level is reached where specific "fundamental activities" for achieving the subgoals can be identified-for example, installing ultrasonic motion detectors in a certain room or performing random-sampling inventories of items in a vault. A synthesis stage follows in which related safeguards activities are identified, perhaps by use of an

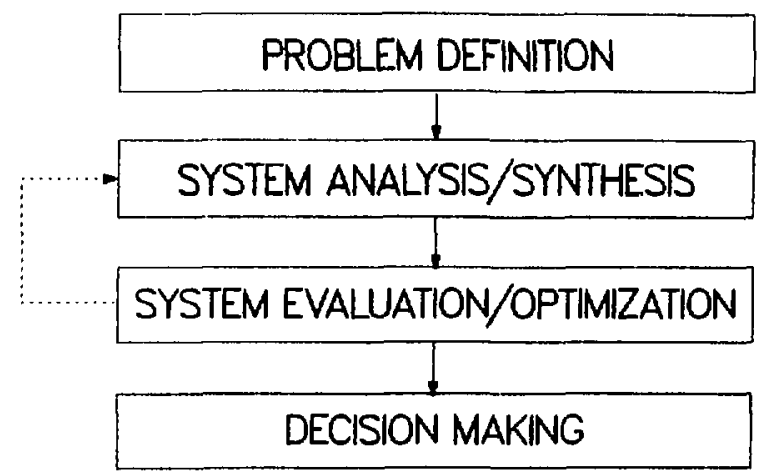

Fig. 45. Phases in the design and evaluation of an integrated safeguards system. interaction matrix such as that shown in Fig. 46. These related activities are then aggregated into subsystems, and a functional integration of activities is performed where possible to improve system efficiency.

The system analysis/optimization phase seeks to determine whether the safeguards system design produced in the analysis/synthesis stage actually achieves the performance goals defined in the problem-definition phase by estimating probable safeguards system performance in detecting and interrupting adversary scenarios constructed from the list of possible adversary actions determined in the problem-definition phase. If deficiencies are found in the safeguards system's responses to these scenarios, then another iteration of the system analysis/synthesis phase must be made. Such an iteration also may be required if the system performance is acceptable but exceśsive redundancy is detected in the safeguards coverage in some areas.

Because of the conflicting goals, it often is the case that if any satisfactory designs can be found, two or more reasonably satisfactory designs having somewhat differing strengths and weaknesses will emerge from the design process. The most common example is identifying a range of designs with increasing safeguards effectiveness and increasing cost. A choice among such multiple designs constitutes the decision-making phase of the safeguards system study. The final selection of the design may simply be left to the subjective judgment of the decision maker. However, in complex cases, it is

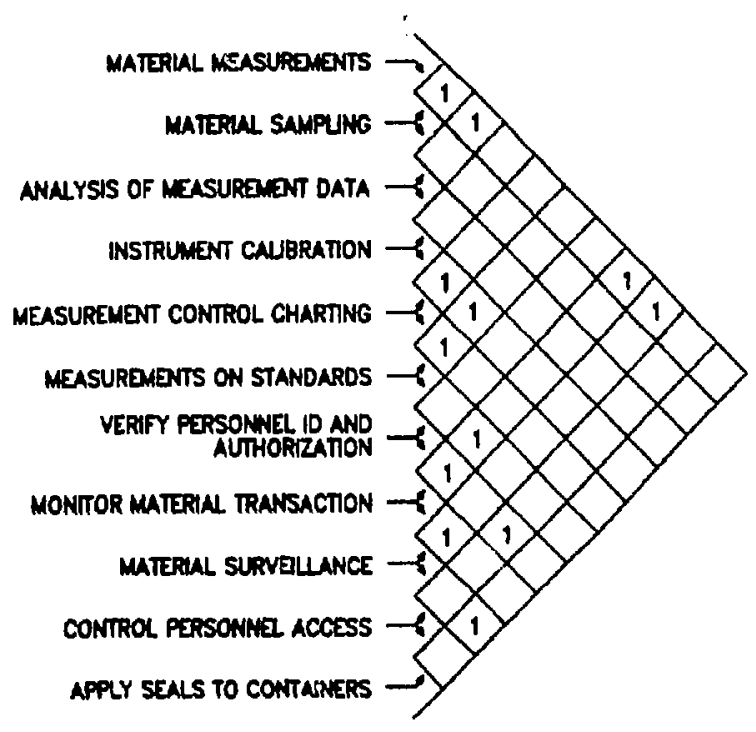

Fig. 46. Information exchange interfaces between fundamental activities. 
usually preferable that a formal decision-making process be used in which well-defined relative weights are assigned to the various performance goals for the system, a utility function is constructed, and the design that yields the highest value for the utility function is selected for implementation. In the report, we provide a brief introduction to the use of these formal decisionmaking methods.

To illustrate the use of the design and evaluation procedures, we briefly describe the application of the method to a hypothetical nuclear-materials storage vault. The methodology has subsequently been applied to evaluate and recommend modification of the tentative design for an actual SNM storage facility to be built at the Los Alamos Plutonium Processing Facility.

The usefulness of the method would be greatly increased if a program guiding the designer through the design process could be impiemented on a microcomputer, and we hope to develop such a program.

\section{B. MC\&A Technology Development.}

MC\&A methods presently implemented at DOE facilnties usually are derived from earlier research. In recognition of this fact, DOE/OSS has continued to fund and encourage research into statistical methods and systems-design methods with the expectation that this work will ultimately find application in operating facilities.

1. Volume Calibration (A. Goldman, Q-4). A current facility-support activity at Savannah River is directed at improving materials accounting for the FB-line process. For this purpose we have constructed process and measurement models and applied them in studying the effect of improved measurement methods and new accounting strategies on FB-line accountancy (Part I, Section I.M). Because volume measurement errors for large tanks in this process are significant contributors to the materials-balance uncertainty, we have been particularly concerned with developing improved error models for these tanks.

These error models are the calibration equations relating an observed liquid level in a tank to the volume of liquid in the tank. For example the expression $y=a+$ $\beta x+\varepsilon$ could represent a calibration equation in which $x$ is the actual volume of liquid, $y$ is the observed level, $\varepsilon$ is the error in observing the level, and $\alpha, \beta$ are parameters determined from a calibration experiment in which controlled amounts of liquid are added to the tank and successive levels observed. We have compared several models of this type in terms of their agreement with experimental data from the FB-line with the purpose of selecting the most appropriate model. The results of this comparison will contribute directly to the improvement of FB-line accountability.

2. An Application of Artificial Intelligence (AI) to Materials Accounting (D. Stirpe, Q-4). Many systems analyses studies require an evaluation of the sensitivity of a materials accounting system to materials loss. Previous evaluations have included generic facilities, operating facilities such as the Savannah River FB-line and the Portsmouth GDP, and facilities in the conceptual design stage such as the Savannah River US Naval FMF. In each case, the uncertainty of a materials balance is derived from rather complicated algebraic calculations that result in an expression for the variance of the materials balance. Because the derivation of this expression involves symbolic rather than numerical calculations, it has previously been done by hand, which can be laborious and time consuming. However, recent interest in applying AI methods to nuclear safeguards has lead to computerization of these symbolic calculations.

Automation of the variance-formula calculation is based on the AI computer program MACSYMA, a large, interactive program designed to assist scientists and engineers in solving mathematical problems. It has a wide range of algebraic-manipulation capabilities that work on symbolic inputs and yield symbolic results as well as an extensive numerical subroutine library and plotting package.

We have used MACSYMA to express the materials balance variance as a function of the individual variances of measurements appearing in the materials balance equation. The calculation is complicated by the presence of correlations between measurements, which must be accounted for in the final variance expression. By phrasing the problem in terms of a matrix operator applied to vectors of coefficients that depend on the measurements, the matrix manipulation features of MACSYMA are applied to find a symbolic variance expression.

This project represents just one of several potential uses of AI techniques in nuclear safeguards. Other possible applications include

(1) an expert system that serves as consultant for guiding inspector activities,

(2) an automated vulnerability assessment using an AI computer program that seeks weak points in a facility's safeguards and security system, and

(3) intelligent interfaces between users and large accountancy data bases to facilitate data input and data manipulation. 
3. Evaluation of a New Test for Materials Loss (E. Kern, Q-4). A basic function of materials accounting is the analysis of a sequence of materials balances to detect evidence of materials loss. This analysis may be formulated as a decision process in which an indicator of materials loss such as the often-used cumulative sum (CUSMU) of materials balances is compared with a decision threshold, and materials loss is indicated when the threshold is exceeded. Our research into such test procedures has provided the basis for materials accounting studies of generic nuclear facilities, for the experiments at the Allied-General Barnwell plant to study near-real-time accounting in a reprocessing facility, and for implementation of accounting systems in operating facilities such as the Savannah River FB-line.

As a result of our bilateral relationship with the Nuclear Research Center at Karlsruhe, FRG, we are familiar with the Center's research in statistical tests. One test of particular interest is the residual MUF algorithm, which is based on the sequence

$$
M_{U F R}=M U F_{i}-E\left(M U F_{i} \mid M U F_{i}, \ldots, M F_{i-1}\right) \text {, }
$$

where MUF $_{\mathrm{i}}$ is the observed material unaccounted for in period $i$, and $E\left(M U F_{i} \mid M U F_{1}, \ldots, M U F_{i-1}\right)$ is the expected value of $M U F_{i}$ given the previous values of MUF. The test statistic CUMUFR ${ }_{i}$ is defined as

$$
\text { CUMUFR }_{i}=\sum_{j=1}^{i} \frac{\text { MUFR }_{j}}{\sigma_{j}},
$$

where $\sigma_{i}$ is the standard deviation of MUFR decision that materials loss has occurred is made when CUMUFR $_{\mathrm{i}}$ crosses a decision threshold that depends on the allowable false-alarm probability.

We have compared the performance of this test procedure with the CUSUM test for a simple process model, several combinations of random and systematic measurement-error variances, and a range of diversion scenarios. The results indicate that, in general, the test for materials loss using the CUMUFR statistic is more sensitive than is the CUSUM test. 


\section{PART 4: INTERNATIONAL SAFEGUARDS}

\section{ENRICHMENT PLANT SAFEGUARDS}

\section{A. Portsmouth GCEP}

The Portsmouth GCEP, under construction near Piketon, Ohio, has been selected by the IAEA for international safeguards inspection. The major goals of the IAEA are to assure that the facility is not misoperated to produce HEU and to verify a materials balance closure around the facility.

Participants in the Hexapartite Safeguards Project (HSP), in which the United States and the IAEA were active, agreed that a way to address the first goal is to allow IAEA inspectors limited access to the process building cascade hall on an unannounced basis, but without continuous inspector presence. Members of the group also agreed that the second goal can be addressed "by providing the inspectorate(s) the opportunity to verify the feed, product, and tails before they are fed to or shipped from the plant." The HSP participants agreed that appropriate detection goals are $75 \mathrm{~kg}$ of contained ${ }^{235} \mathrm{U}$ in 1 year. The materials balance verification will be based on routine inspection visits.

Methods now used by the IAEA for ad hoc inspection of enrichment facilities could pose an unacceptable impact on the operations of the full 8.8-million-SWUper-year GCEP. As a result of technical studies supported by the $\mathrm{DOE}$ and directed by UCC/ND, advances in technology have been made that can provide more effective safeguards while reducing the impact on facility operations. ${ }^{44-46}$

The system described here was designed by a team including the DOE, UCC-ND, Goodyear Atomic Corporation, BNL, Los Alamos, and Sandia National Laboratories. Fluor Engineers, Inc., Irvine, California, is the architect/engineer for the safeguards system facility.

Los Alamos has primary responsibility for the enrichment monitors, the integrated data acquisitions system, and the limited-frequency unannounced access (LFUA) strategy tasks. Los Alamos also provides technical assistance in support of the DOE Enrichment Safeguards Program Review and has continued development of area neutron-monitoring technology.
1. Enrichment Monitoring System (J. K. Sprinkle, Jr., R. B. Strittmatter, D. L. Garcia, J. N. Leavitt, R. Siebelist, and R. W. Slice, Q-1; A. L. Baker and D. Stirpe, Q-4). IAEA verification of the facility's nuclear materials balance requires verification of major $\mathrm{UF}_{6}$ flows, minor flows, waste streams, and $\mathrm{UF}_{6}$ inventories. The major uranium flows are $U_{6}$ in the form of feed, product, and tails. For a one-building, 1.1-millionSWU-per-year GCEP, estimated annual flows include 193 10-ton and 14-ton cylinders of feed, 43 10-ton cylinders of product, and 13510 -ton cylinders of tails. To satisfy the state system of accountability and control, the facility operator will measure the mass, the uranium mass fraction, and the ${ }^{23 S} U$ weight fraction of the cylinder contents. These data are declared to the IAEA and verified by their inspection teams.

The enrichment monitoring system was designed to provide the IAEA with continuous, verifiable, measurements of the gas-phase feed, product, and tails of $\mathrm{UF}_{6}$ streams. ${ }^{44}$ The logical design of the system is shown in Fig. 47. The enrichment monitor processor (EMP) computer is a front-end processor for the integrated data acquisition system (IDAS) and verifies data transmissions from the enrichment monitors and the integrated monitoring system (IMS). The IMS functions as a secured tamper/intrusion detection system. The EMP records the verified data it has received on a disk and transmits it to the IDAS. The IDAS stores the data on its own disk, performs a sequential analysis, and checks for irregularities. Thus, there is a continuous check on the operation of the enrichment monitoring system.

An inspectorate facility provides the instruments and the computers with a benign tamper-indicating en vironment that is essential for the reliable operation of the safeguards system and localizes the inspector's activities, which reduces the operator's risk of disclosing sensitive centrifuge technology.

The four enrichment monitors will be located in the inspectorate facility, a separate building adjacent to the feed-and-withdrawal building. One monitor is connected to each of the feed, product, tails, and spare tails lines in the interconnecting process pipeway (IPP) that connects the feed-and-withdrawal building with process 


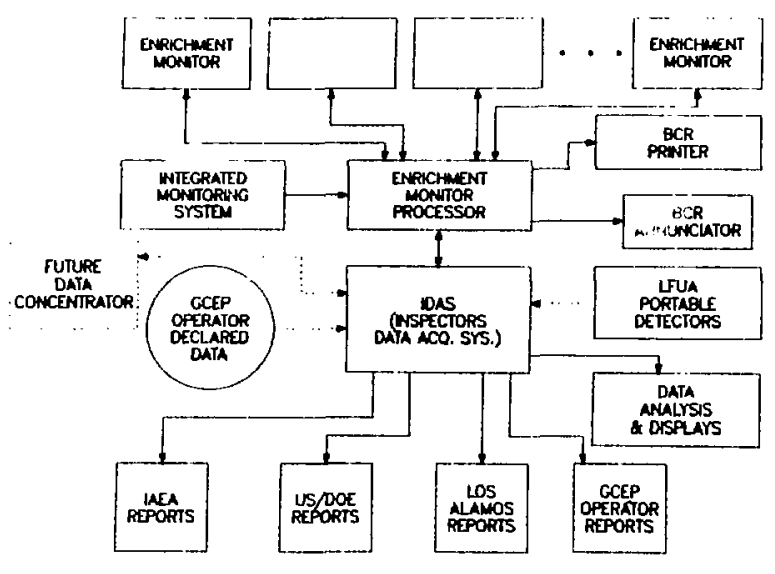

Fig. 47. Logic diagram of the international safeguards system for GCEP.

buildings 1 and 2 . The sampling line includes compressor pumps to provide the pressure needed at the monitors to provide the desired assay accuracy. The inspectorate facility (Fig. 48 ) is divided into four areas: two equipment rooms, a monitor room, and an operations room for computers and desks. The monitor room and computer room will be sealed and use intrusion monitoring equipment designed by Sandia National Laboratories.

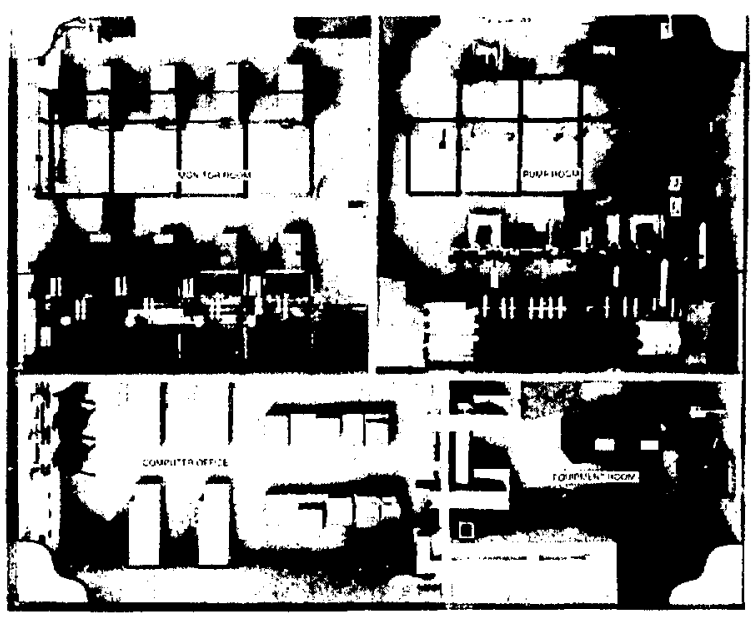

Fig. 48. Plan view of the inspectorate facility for the international safeguards system at the Portsmouth GCEP. The monitor and pump rooms show additional equipment that would be installed for monitoring $\mathrm{UF}_{6}$ from future plant expansions. a. Gas-Phase Enrichment Monitor Subsystem. The gas-phase enrichment is determined by combining measurements of ${ }^{235} \mathrm{U}$ and total uranium concentrations. The ${ }^{235} U$ concentration is determined from a measurement of the $185.7-\mathrm{keV}$ gamma rays emitted from the decay of ${ }^{235} \mathrm{U}$, and the total uranium concentration is determined by measuring the transmissions through the $\mathrm{UF}_{6}$ gas of $60-\mathrm{keV}$ gamma rays from an external ${ }^{241} \mathrm{Am}$ source. ${ }^{47,48}$ Tests in an operating plant environment have achieved assay accuracies of $0.5 \%$ relative at an enrichment of $0.9 \%$.

The GCEP enrichment monitor design (Fig. 49) is based on previous in-plant experience with a prototype monitor and on facility and IAEA operation constraints. ${ }^{48}$ The instrument electronics are in the front cabinet, which also houses the detector dewar, linepower conditioner, and interface junction boxes. The rear cabinet is heated and houses the measurement chamber, gamma-ray shielding, radioactive sources, measurement control shutter devices, $\mathrm{UF}_{6}$ piping, flow meter, and heater elements. An insulated port in the heated enclosure accepts the detector snout. All nuclear electronics are standard commercial NIM modules,

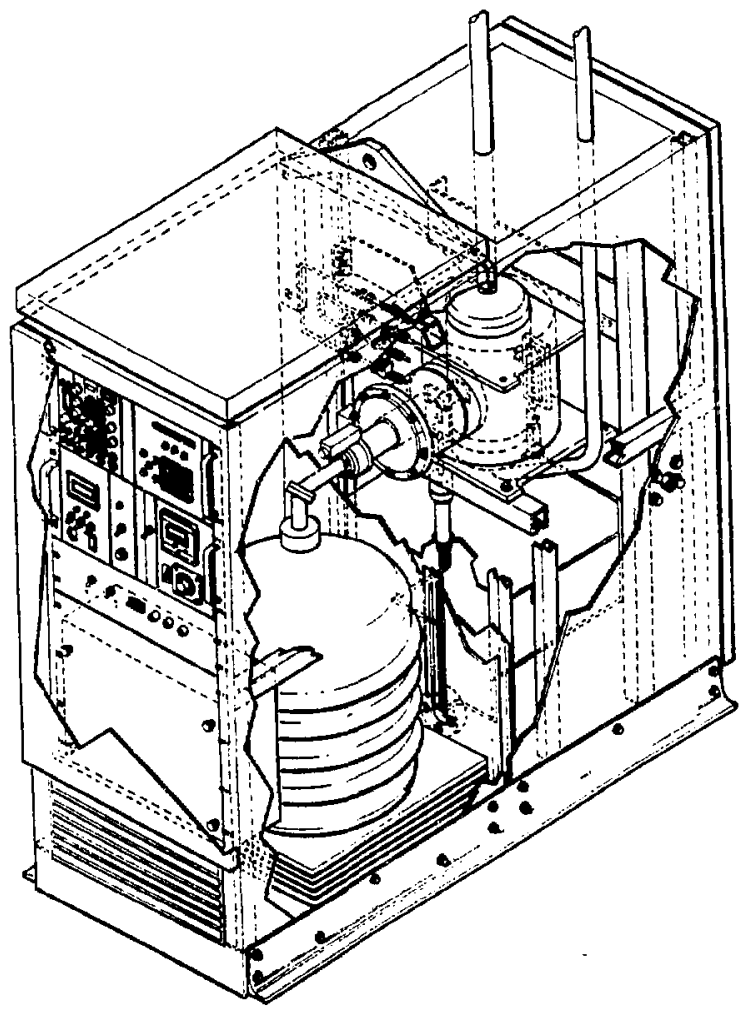

Fig. 49. GCEP gas-phase UF 6 enrichment monitor. 
which permit easy on-site maintenance by replacement. The detector is a 51 -mm-diam, 15-mm-thick, highperformance germanium (HPGe) planar type with an integrally mounted 3.5 -cm-thick lead back shield. The Los Alamos-designed programmable data acquisition and control (PDAC) module provides for automated data acquisition, data analysis, control, and data transmission functions.

A prototype GCEP enrichment monitor was fabricated and delivered to the Oak Ridge GDP during 1983. It was installed at the Withdrawal Desublimation Test Facility (WDTF) where a special slipstream test loop has been constructed. We will use information gained from the prototype to improve the final GCEP enrichment monitor design, which is to be certified for construction by March 1984.

b. Computer Subsystem. We are developing the safeguards data system to

- record data continuously from the enrichment monitors,

- store information indicating possible attempts to thwart the system,

- support LFUA activities,

- support GCEP operators' declarations of materials accounting information, and

- enable the inspector to perform analyses of the data at the GCEP site. ${ }^{49}$

The primary requirement is a computer system that operates continuously and reliably during unattended periods. Additional requirements include the capacity for future expansion and operation by inspectors having minimal computer experience. To meet these unique demands we have chosen a distributed system of computers that will facilitate task separation, allowing responsive and reliable software.

The IDAS computer is the primary interface with the IAEA inspector. It will receive, store, and examine enrichment data from the EMP. The redundant data storage in the EMP and the IDAS provides a back-up capability. The IDAS also will have the capability to read data stored in the portable monitors used for the LFUA inspection strategy. These data will be recorded on a disk file and examined for anomalies. In addition, the GCEP operator's declarations of $U F_{6}$ cylinder data will be transferred into the IDAS, which will give the inspector the capability to examine any portion of the data received and generate reports or graphs. On request, the IDAS will prepare reports for DOE, GCEP operators, and the system developers.

The IDAS is configured as a Digital Equipment Corporation VAX $11 / 730$ computer system that includes a removable RL02 disk pack and a fixed RA80 disk. The system inciudes a VT125 graphics terminal and a printer/keyboard terminal for printed output. Communications will be through RS422 communication lines in standard ASCII format. Delivery of the IDAS computer system to Los Alamos is expected in early 1984 , when the $50 \%$ software design review also will take place.

c. Attributes/Variables Measurements and IAEA Derection Sensitivity. As part of our development of an unattended system for IAEA use in monitoring the inrichment of the feed, product, and tails streams at the Portsmouth GCEP, we are evaluating IAEA strategies for using enrichment monitors and laboratory analyses to verify materials balances. We have determined the detection sensitivity of three of the several verification procedures available to the IAEA that use the major streams (feed, product, and tails) at GCEP. In the first procedure, we use an in-line enrichment monitor as an attributes measurement of the ${ }^{235} \mathrm{U}$ concentration to determine the number of samples that should be sent to the IAEA Seibersdorf Analytical Laboratory for more precise variables measurements. In the second procedure, we use an in-line enrichment monitor for both attributes and variables measurements. In the third procedure, the number of variables cylinder-mass measurements is reduced by using a relatively imprecise attributes measurement to determine the sample sizes of feed, product, and tails cylinders to be remeasured with more precise instruments. Using generally accepted values of measurement-error standard deviations for the different attributes and variables measurement instruments, we estimate detection sensitivities for a oneyear balance period of 345,202 , and $202 \mathrm{~kg}^{235} \mathrm{U}$, respectively, for these three potential verification procedures.

2. Inspection Strategy (J. C. Pratt, D. A. Close, and K. V. Nixon, Q-2). the LFUA inspection strategy, proposed for IAEA implementation at GCEP, comprises unannounced visits to the cascade hall where the inspector will observe the equipment and measure the gamma-ray output of randomly chosen pipes between the cascade boundary and the train headers using portable high-resolution gamma-ray detectors. Each of these measurements will be made for a short time only. Because these measurements are to verify normal facility operation, rather than to confirm the announced enrichment, and to allow inspection of more pipes in a given time, short routine measurements are made to detect the anomalous radiation output characteristic of HEU production. Anomalies detected in the routine activity will be followed immediately by other activities 
to confirm and document a possible facility misoperation.

a. Physical Basis of the Gamma-Ray Measurements. The primary gamma ray of interest is the $185.7-\mathrm{keV}$ gamma ray emitted directly from ${ }^{235} \mathrm{U}$. Because this gamma ray is the result of a nuclear decay process, the emission rate is independent of the chemical configuration or physical motion of the uranium. It is detected by a high-purity germanium detector collimated to view only the pipe under inspection. Because the detector response is a function of the entire collection of ${ }^{235} \mathrm{U}$ atoms in the pipe, the quantity of ${ }^{235} U$ is measured rather than uranium enrichment. An enrichment measurement would require an independent measurenient of the ${ }^{238} \mathrm{U}$ present or the total uranium present. Unfortunately, the ${ }^{238} \mathrm{U}$ direct emissions at $49.7 \mathrm{keV}$ are extremely weak, and the 1001 - and $766-\mathrm{keV}$ gamma rays normally associated with depleted uranium come from the ${ }^{234 \mathrm{~m}} \mathrm{~Pa}$ daughter, which is nonvolatile and remains almost entirely in the feed cylinder. Consequently, direct gamma-ray measurement of the ${ }^{238} \mathrm{U}$ present is impracticable. Interpretation of the ${ }^{235} U$ signal as an approximate relative indicator of enrichment therefore requires the assumption of constant total uranium under observation. There are two ways that this assumption can be violated. The first is an increase or decrease of the pressure in the pipes, which will change the quantity of gas under observation. Consequently, assumption of constant uranium in the gas phase requires that either the pressure be independently measured or that verifiable details of the plant operation be invoked to demonstrate that pressure adjustment sufficient to hide the high-enrichment anomaly signal is technically difficult. If the degree of technical difficulty is sufficient, the assumption of inspection effectiveness against credible levels of diversion effort can be justified.

With the assumption of constant gas-phase uranium and interpretation of the ${ }^{235} \mathrm{U}$ gamma-ray count rate as a relative enrichment indicator, there remains the problem of extra uranium deposited in the pipes. The ${ }^{235} \mathrm{U}$ in the deposit will contribute to the total ${ }^{235} \mathrm{U}$ signal and has been observed to provide a significant fraction of the $185.7-\mathrm{keV}$ count rate. Deposit count rates can be isolated by separate measurements of the pipe in operation and under vacuum. Whereas such measurements are more appropriate to research activities than to routine inspections, this is a potential method to resolve an anomaly produced by other routine activities. Becruse deposits have been observed that produce two to three times the signal from the gas with the resulting signal three to four times that expected from a clean pipe with the same amount of gas, it is helpful to think in terms of a deposit-concentration unit that gives a count rate equal to that of the gas-phase uranium alone. Then a pipe with a twice-gas deposit of $2.85 \%$ enrichment product material would look like a relative enrichment measurement of $8.55 \%$. If the anomalous, misoperation signal is defined to be that produced by a relative enrichment of $20 \%$, there is considerable difference. The signal from the apparent $8.55 \%$ enrichment for a three-minute measurement would be a few hundred counts, and the difference between the two can be determined to a statistically significant level in a few minutes. Thus, the separation between the normal $2.85 \%$ production enrichment and an anomaly defined at $20 \%$ is sufficient to allow considerable deposit before the routine inspection activity would produce false positive results.

The ${ }^{238} U$ in the deposit inside the pipes decays into the daughters shown in Table XI. The 63.3-keV gamma ray from the ${ }^{234} \mathrm{Th}$ is in equilibrium with the ${ }^{238} \mathrm{U}$ parent

\begin{tabular}{|c|c|c|c|c|c|}
\hline \multicolumn{6}{|c|}{ TABLE XI. Initial Decays of Uranium Series } \\
\hline Decay & Isotope & $\mathbf{T}_{1 / 2}$ & Comment & $\begin{array}{c}\text { Gamma } \\
\text { Rays } \\
\text { (keV) }\end{array}$ & $\begin{array}{c}\text { Relative to } \\
185.7 \mathrm{keV} \\
\text { of Natural } \\
\text { Uranium } \\
\end{array}$ \\
\hline $\mathbf{0}$ & ${ }^{238} \mathrm{U}$ & 4.47E9 y & Primarily $\alpha$ & 49.4 & $\ldots$ \\
\hline 1 & ${ }^{234} \mathrm{Th}$ & $24.1 \mathrm{~d}$ & $\begin{array}{l}\text { Doublet } \\
\text { Doublet }\end{array}$ & $\begin{array}{l}63.3 \\
92.5\end{array}$ & $\begin{array}{l}1-10 \\
1-10\end{array}$ \\
\hline 2 & ${ }^{234} \mathbf{P a}$ & $6.7 \mathrm{~h}$ & Metastable & 766 & -- \\
\hline 3 & ${ }^{234} \mathbf{U}$ & 2.45E5 y & Primarily $\alpha$ & $\begin{array}{l}1001 \\
53.2\end{array}$ & $\begin{array}{c}0.1-1 \\
\ldots . . .\end{array}$ \\
\hline
\end{tabular}


afier approximately 90 days and can be used as an approximate measurement of the uranium in the deposit. It is approximate for two reasons. The first is that recent deposits will not have the daughters in equilibrium as shown in Fig. 50, where the time history of the signal from a pipe is simulated for an arbitrary deposit collection scenario. The ${ }^{234} \mathrm{Th}$ signal lags behind the ${ }^{235} \mathrm{U}$ signal on sudden accumulation of the deposit but restores its relationship as the daughters come into equilibrium. The uranium with daughters not in equilibrium is that fraction of the entire deposit deposited in the last few weeks; with continuous deposition this fraction becomes smaller. The second reason that the $63.3 \mathrm{keV}$ gives only an approximate indication of the deposit presence is the apparent transport of ${ }^{234} \mathrm{Th}$ in the gat. Figure 51 shows the ${ }^{235} \mathrm{U}$ signal and the ${ }^{234} \mathrm{Th}$ signal after a bend in the Centrifuge Plant Demonstration Facility (CPDF) product pipe. Because the bend followed a straight section approximately 30 meters long, the variation in the thorium signal suggests that the thorium was transported in the gas and deposited after the bend.

Because similar behavior was observed in the CPDF feed pipe outside the vaporizer, positions for pipe measurements must be chosen with this effect in mind. We suggest that inspectors, although allowed to measure anywhere outside the cascades, mark carefully chosen places on each pipe to allow direct comparison between measurements from several visits. All measurements, both results and entire spectrum, will be recorded on tape in the cascade hall and subsequently transferred to

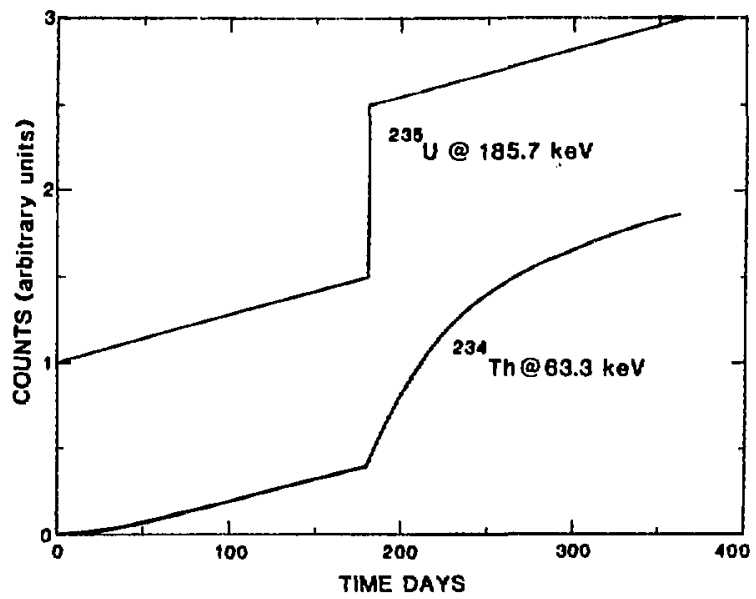

Fig. 50. Computer model of the relative behavior of $185.7-\mathrm{keV}$ (uranium-235) and 63.3-keV (thorium-234) count rates, for the following accumulation scenario. Deposit builds uniformly for 180 days, rate of one gas-equivalent in 360 days. Sudden deposit of one gas-equivalent at day 180. Deposit builds uniformly as before. a data base in the IDAS, where the accumulation of the deposit will be monitored and the relative behavior of the ${ }^{235} \mathrm{U}$ and ${ }^{234} \mathrm{Th}$ signals can be collected for each class of pipe. Figure 52 shows such a comparison from the Component Test Facility (CTF) product pipes. The ${ }^{234} \mathrm{Th}$ signal at both 63.3 and $92.4 \mathrm{keV}$ assumes a stable ratio with the ${ }^{235} \mathrm{U}$ signal, leading to the approximate gas isolation calculation, ${ }^{235} \mathrm{U}$ gas signal + rate (185.7)$k^{*}(63.3)$, where $k$ is the empirical slope of a graph like Fig. 52 for each class of pipes (extraction of the 92.4-keV peak is complicated by the uranium $x$-rays nearby, so only the $63.3-\mathrm{keV}$ peak is used).
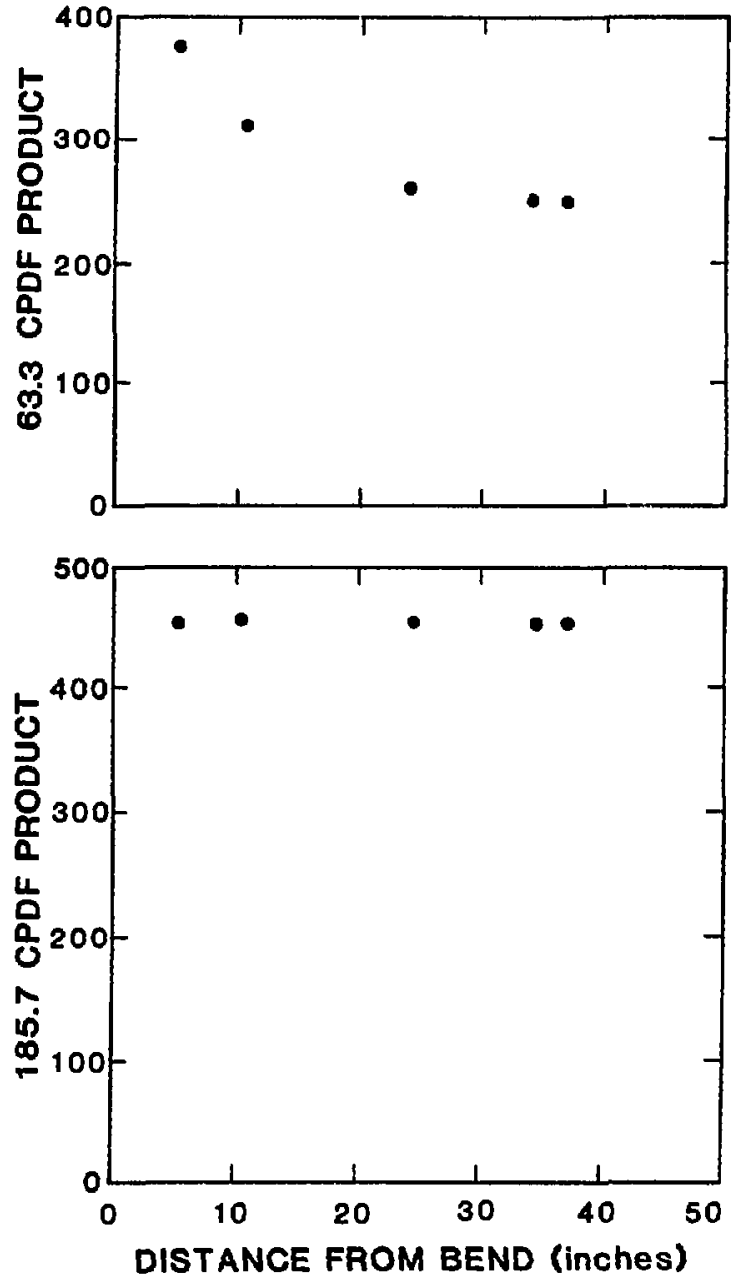

Fig. 51. The Centrifuge Production Demonstration Facility (CPDF) product pipe has a long straight section, approximately 30 meters long, followed by a bend. The figure shows the $63.3-\mathrm{keV}$ (thorium-234) and $185.7-\mathrm{keV}$ (uranium-235) photopeak count rates as a function of position after the bend. The units shown are arbitrary. 

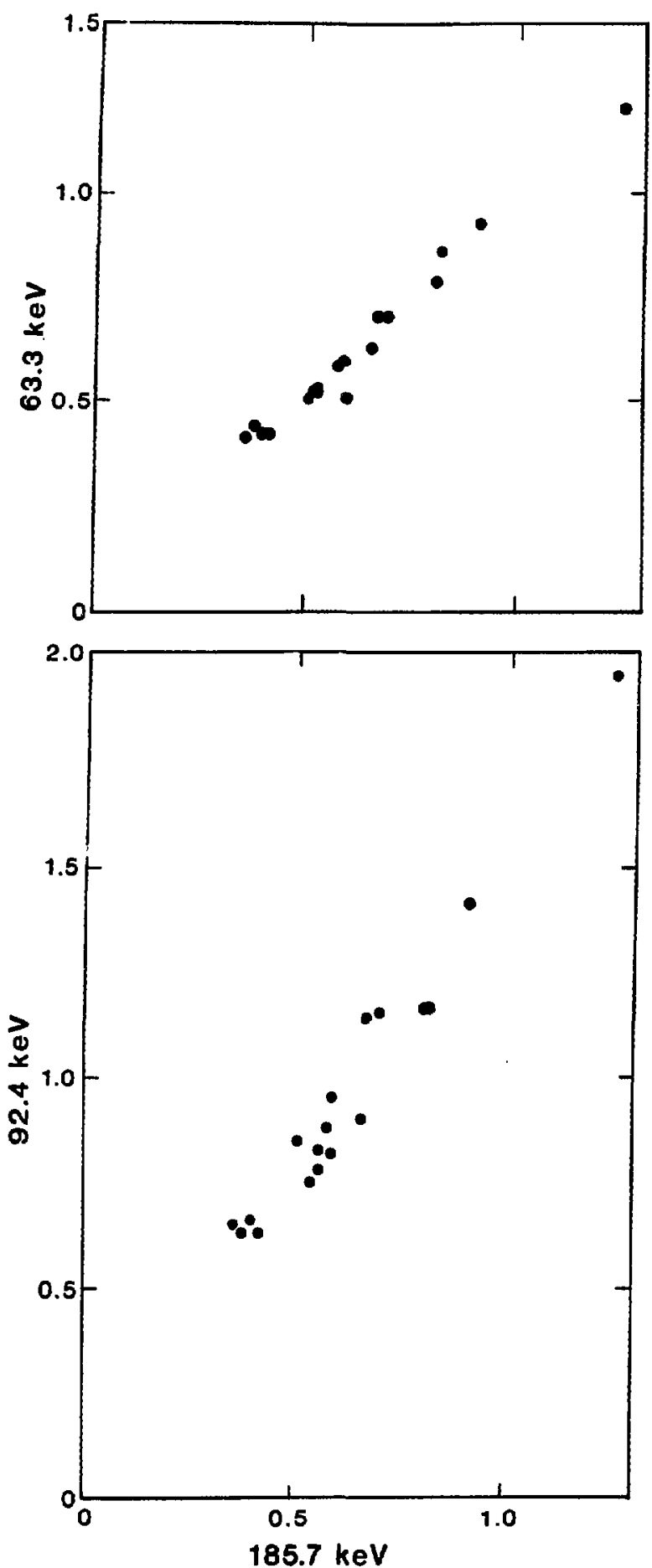

Fig. 52. Centrifuge Test Facility (CTF) product pipe deposit photopeak count rates for $185.7-\mathrm{keV}$ gamma ray (uranium-235), 63.3$\mathrm{keV}$ gamma ray (thorium-234), and $92.4-\mathrm{keV}$ gamma ray (thorium-234). Units shown are counts per second. Error bars of approximately $5 \%$ are omitted for clarity.
Thus, the routine activity is a real-time inspection of the current state of randomly chosen pipes in the facility. Approximate removal of the deposit contributions provides a more accurate estimate of the ${ }^{235} \mathrm{U}$ signal from the gas-phase uranium, and interpretation based on the assumption of a constant quantity of gasphase uranium makes this an approximate relative enrichment measurement.

b. Measurement Activities. The routine activity aims at verification that individual pipes do not exhibit the anomaly of elevated ${ }^{235} \mathrm{U}$ signals. The acquired spectra are analyzed immediately by firmware in the commercial portable battery-powered pulse-height analyzer that contains the amplifier and power supplies necessary to operate the high-resolution detector. The spectrum, together with the information necessary to document the measurement in the IDAS data base, is automatically recorded. This use of the analyzer as a small portable computer provides an immediate interpretation of the spectrum with minimum inspector effort. The firmware extracts the photopeaks for the ${ }^{235} \mathrm{U}$ and ${ }^{234} \mathrm{Th}$, calculates the approximate gas-phase ${ }^{235} \mathrm{U}$ signal, and decides if this signal is anomalously high. The decision process, called the sequential probabilitv ratio test, requires a series of spectrum measurements, but only the minimum amount of data necessary to make the decision with a specified statistical reliability is taken. After each spectrum is required and the ${ }^{235} \mathrm{U}$ gas contribution calculated, the results from all the spectra in the sequence are compared with the two hypotheses of worst-case expected normal operation and facility misoperation.

The ratio of the probability that the sequence of observations could have been produced by facility misoperation to the probability that the sequence could have been produced under normal operation is compared with a pair of threshold values computed from the tolerable false-alarm probability and the desired detection probability. In this case, the false-alarm probability is established as $1 / 1,000,000$, and the desired detection probability is $95 \%$. The comparison of the probability ratio with these two values results in three possibilities: one of the two hypotheses can be accepted if the ratio value is above or below both thresholds or, if the ratio value falls between the two thresholds, no decision is made and another spectrum is taken. Spectrum acquisition continues until the combined statistics of the entire sequence spectra permits a decision. As each new spectrum in the sequence is acquired, it is added to an accumulated total spectrum for that sequence; the total is the spectrum recorded in the data base.

Thus, the portable computer facilitates a sophisticated statistical decision-making process that reduces the inspector's effort and speeds the measurement. Follow-up action to clarify anomalous results can take place immediately. 
c. Equipment. The gamma-ray detector is a planar high-purity germanium detector with a front surface area of $20 \mathrm{~cm}^{2}$ and a $1-\mathrm{cm}$ thickness. It is mounted in a portable all-attitude dewar that contains enough liquid nitrogen for 12 hours of measurements. A tungsten disk is located behind the detector (Fig. 53), and a tungsten collimator, lined with brass to prevent the tungsten $\mathrm{x}$ rays from interfering with the ${ }^{231} \mathrm{Th}$ gamma at 82.4 $\mathrm{keV}$, is attached outside the cryostat. The outer end of the collimator is machined to fit the principal pipe size in GCEP, and, although the collimator is retained on the cryostat, it can rotate to measure either vertical or horizontal pipes. The detector stand that clamps to the pipe is shown in Fig. 54.

The portable analyzer/computer is a Canberra Series 10 equipped with the factory option of an extra card slot and an extra display processor card (SERF 1008) to carry the firmware for the inspection activity. The firmwars allows the inspector single-key choices for the information needed to define the measurement type (train, cascade, pipe) for the IDAS data base. The inspector has the option of viewing graphical representations of the automatic photopeak extraction to verify that the decision process is working with reasonable data. The inspector also can view plots of the expected probability distributions and the current estimate of the distribution of the ${ }^{235} \mathrm{U}$ signal being measured. Thus, the inspector can function as an independent, real-time monitor and critic of the automatic decision process.

The analog output of the analyzer is strong enough to drive two external recorders simultaneously, which allows the firmware to make two simultaneous originals of the inspection record. Thus, the inspector and the

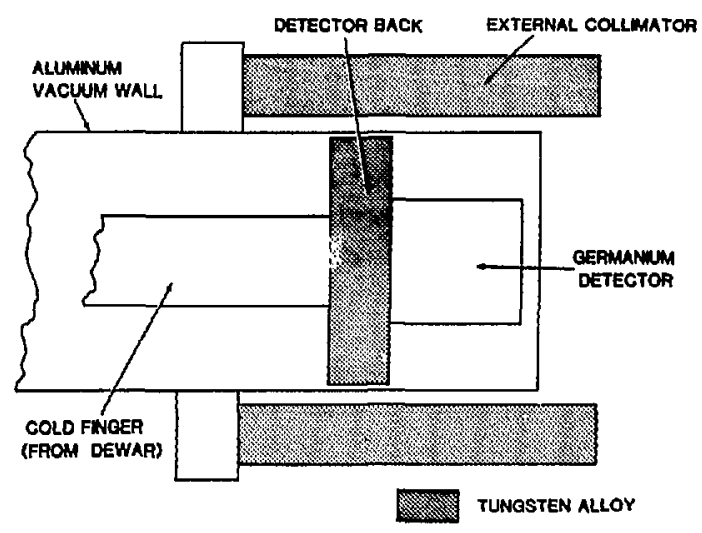

Fig. 53. Cross section of detector and cryostat showing internal tungsten disk and external tungsten collimator.

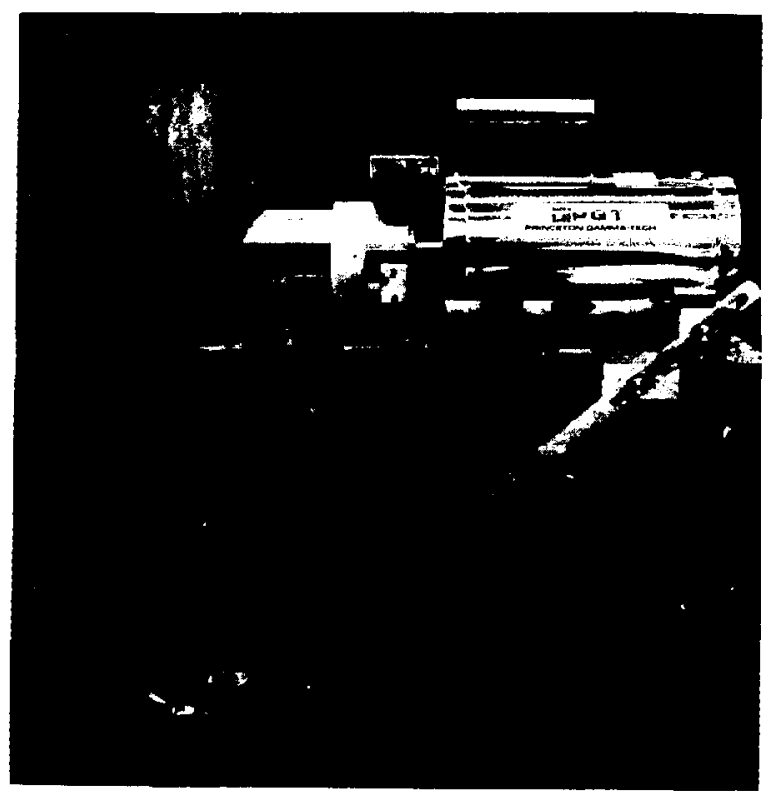

Fig. 54. Photograph of detector, mounted on vertical pipe by clampon detector holder. The GCEP tails pipe and the cascade recycle pipe at the tails end of the cascade are vertical pipes.

escort from the host organization can each have an original tape of the inspection results, giving two independent archives.

3. Neutron Area Monitoring: A Nonintrusive International Inspection Strategy for Centrifuge Enrichment Plants (J. E. Stewart, Q-1). Arrays of neutron monitors at fixed positions inside a centrifuge cascade hall can provide the international community with assurance of normal operation and deterrence of misoperation with low cascade-access requirements and little or no facility modification. ${ }^{50}$ Facility misoperation includes producing $U_{6}$ at higher enrichments than declared, and neutron emission from $U_{6}$ is a signature of uranium enrichment. We are developing and evaluating neutron monitors for implementation in GCEP and in the URENCO-Centec separation plant (SP-4) near Almelo, the Netherlands. The emerging technologies represented by these two plants provide interesting contrasts with regard to in-process inventory, treatment of classified data, equipment size, plant layout, and operating philosophy, all parameters that affect a neutron-monitoring system. Figure 55 shows US centrifuges (lower right), URENCO centrifuges (upper left), and a prototype neutron-monitoring system. 


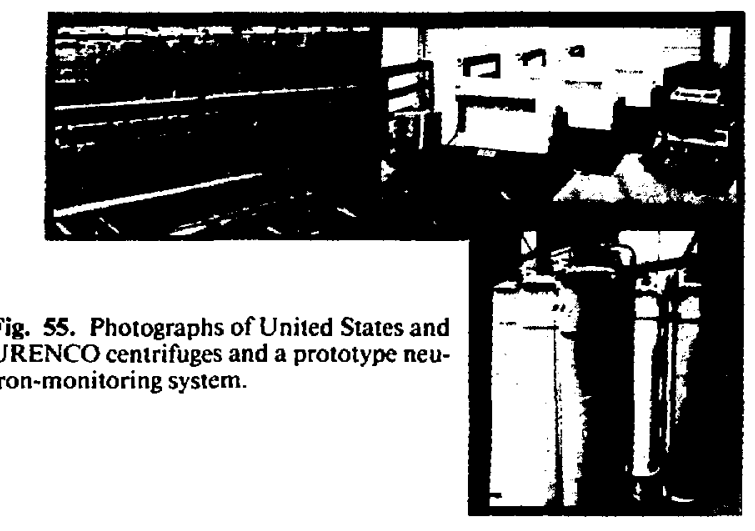

A prototype two-detector system for GCEP was installed at the CPDF at Oak Ridge in August 1982. The two detectors were mounted at the product and tails ends of a single cascade of centrifuges identical to those installed in the first process building (PB1) of GCEP. The system has operated continuously since installation and throughout 1983 . The only maintenance required was cleaning the floppy disk drive mechanism.

At CPDF, in contrast to GCEP, feed and withdrawal operations are conducted near the separation equipment, and elevated neutron signals from feed, product, and tails cylinders are registered routinely by the two detectors. For example, during July a feed cylinder was brought to the autoclave, partially drained, removed, brought back, and drained further. These operations were detected clearly from a distance of approximately $30 \mathrm{~m}$ by one of the neutron detectors.

In collaboration with Union Carbide (the operator of CPDF), we prepared a test plan for (1) continuation of measurement using the two detectors, (2) addition of a third monitor to view the feed vaporization area, (3) addition of a fourth monitor to view the product and tails withdrawal areas, and (4) installation of coaxial cabling between the new detectors and the data acquisition system in the control room. Installation of the two additional neutron detectors will significantly increase the sensitivity to movements of $\mathrm{UF}_{6}$ in the feed and withdrawal areas. Also, the four-detector array will provide valuable data for developing background subtraction methods for the cascade detector responses.

Initial experiments at Almelo using one neutron area monitor in SP-4 and another in a remote nonprocess area were conducted in 1981-1982. Subsequent data analysis ${ }^{51}$ showed that routine cylinder operations in the feed and withdrawal area adjacent to the cascade halls caused significant signals inside the halls; thus, as at CPDF, the need to monitor background from routine feed and withdrawal operations is clear. These experiments were carried out in collaboration with the Netherlands Energy Research Foundation (ECN).

With ECN we did a preliminary evaluation of the effectiveness of a neutron area-monitoring system in 1983, based on experimental data and calculations of neutron fields for various hypothetical scenarios of HEU production. A preliminary conclusion, pertaining only to facilities of the present URENCO design, is that limited arrays of monitors (fewer than 10 monitors per cascade hall) have a low detection for some scenarios of protracted HEU production, but provide high assurance that no large-scale HEU production has taken place between inspections and that no unusually large quantities of $\mathrm{UF}_{6}$ have been introduced into the hall. Thus, continuous neutron area monitoring may reduce the need for frequent inspections inside the cascade area. A paper summarizing results of this analysis of the Almelo-URENCO case was submitted to the 6th ESARDA Symposium on Safeguards and Nuclear Materials Management, held in Venice, Italy, in May 1984.

This technology may have application in the second process building at GCEP. It also may have application in gaseous diffusion plants where the reutron signal is considerably higher; it might, for instance, be used to help the plant operator locate solid deposits held up in the cascade equipment. The movement of irradiated fuel at a reactor or fuel-reprocessing facility might be monitored using similar techniques.

4. Tests at Almelo (R. H. Augustson, Q-1). Safeguarding centrifuge enrichment plants is one of the most difficult technical challenges currently facing, international safeguards, and an international study under the sponsorship of the Hexapartite Group has been addressing the associated problems for approximately 2 years. One important issue is inspector access to the cascade hall. If an inspector is allowed into the hall, what relevant but sensitive measurements can be made? A tour at the Netherlands ECN in Petten has made gamma-ray measurements on cascade equipment at the Almelo centrifuge enrichment facility in an effort to resolve this question. To improve instrument reliability, portability, and data analysis, Los Alamos loaned the group a Davidson portable multichannel analyzer. We are carrying out a similar study at the 
CDPF at Oak Ridge and, in the coming year, will exchange and compare results with the Petten group. Both studies are necessary because the European and US cascades are operated differently. We hope the studies may lead to a statement regarding what inspection measurements are possible and acceptable in the cascade halls.

B. Compact ${ }^{252} \mathrm{CfShuffler}$ for $\mathrm{UF}_{6}$ Measurements (H.O. Menlove, S. C. Bourret, D. C. Garcia, and D. L. Garcia, Q-1)

We have a developed a technique to nondestructively assay small product samples of $U F_{6}$ from enrichment plants. Typical samples consist of 5 to $10 \mathrm{~g}$ of $\mathrm{UF}_{6}$ in small metal cylinders or Teflon tubes. The $\mathrm{UF}_{6}$ is distributed nonuniformly in the container, making gamma-ray assay difficult.

Because the ${ }^{252} \mathrm{Cf}$ shuffler method has been used successfully for several years to assay bulk samples of uranium, we designed a compact shuffler that can be moved easily to different measurement locations for the assay of small (subgram) quantities of uranium or mixed oxide (MOX). Figure 56 shows the instrument's source transfer system, neutron shield, and detector. The detector is an inventory sample coincidence counter (INVS), ${ }^{52}$ which was developed previously for

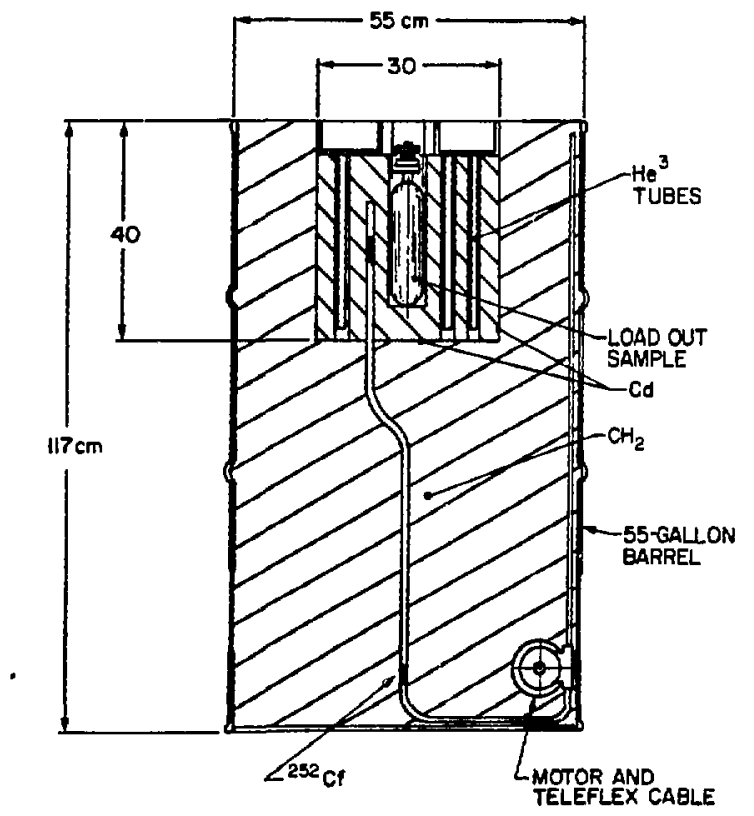

Fig. 56. Schematic diagram of compact ${ }^{252} \mathrm{Cf}$ shumfer for $U F_{6}$ assay.
IAEA inspectors to measure small plutonium samples, and usually operates with the shift-register coincidence electronics ${ }^{53}$ to measure the spontaneous fission rate from $\mathrm{PuO}_{2}$ powder and pellets and plutonium nitrate. When this passive coincidence system is combined with the ${ }^{252} \mathrm{Cf}$ active-assay shuffler, it can be used for uranium assay as well as MOX and plutonium.

We obtained the compact design of the present shuffler by using a small ${ }^{252} \mathrm{Cf}$ thermal neutron source (about $5 \times 10^{6} \mathrm{n} / \mathrm{s}$ ), a high-efficiency detector (about $37 \%$ ), and close source-sample coupling. The low intensity of the ${ }^{252} \mathrm{Cf}$ source allows handling for brief periods with little or no shielding, and when the ${ }^{252} \mathrm{Cf}$ source is in the barrel (Fig. 56), there is negligible personnel dose. The column of polyethylene between the ${ }^{252} \mathrm{Cf}$ source and the detector gives better neutron shielding per centimeter than in the larger shuffler systems because of "shadow shielding" where the outscatter is greater than the inscatter. Figure 57 shows the measured relative neutron rate in the INVS versus source position in the shield. The rate of neutron dose reduction is a decade for every $10 \mathrm{~cm}$ of polyethylene compared with a typical value of a decade for each $15 \mathrm{~cm}$ in a larger shield.

We completed design and fabrication of the compact shuffler in 1983; testing and evaluation with $\mathrm{UF}_{6}$ samples will take place in the first half of 1984. Later, we will evaluate the system in the hybrid mode for MOX samples by combining the passive coincidence plutonium measurement with the active delayed-neutron rate to solve for both the uranium and plutonium content.

Potential applications of this instrument include $\mathrm{UF}_{6}$ samples taken at an enrichment plant and almost any situation where a small sample is taken of uranium or plutonium materials. It may have application in reprocessing plants to measure highly radioactive samples from process points before the final product stage.

\section{REPROCESSING PLANT SAFEGUARDS}

\section{A. Compact K-Edge Densitometer (L. R. Cowder, S. F.} Klosterbuer, and R. H. Augustson, Q-1)

For several years a K-edge densitometer, fabricated at Los Alamos, has been operating in-line at the reprocessing plant in Tokai-mura, Japan. In response to an IAEA request for an independent means of verifying results from this instrument, we have developed and tested a compact version that uses a portable, computer-based, multichannel analyzer with special software to make possible measurement of the same plutonium solution samples measured by the in-line densitometer. Its unique design allows measurements to be made through 


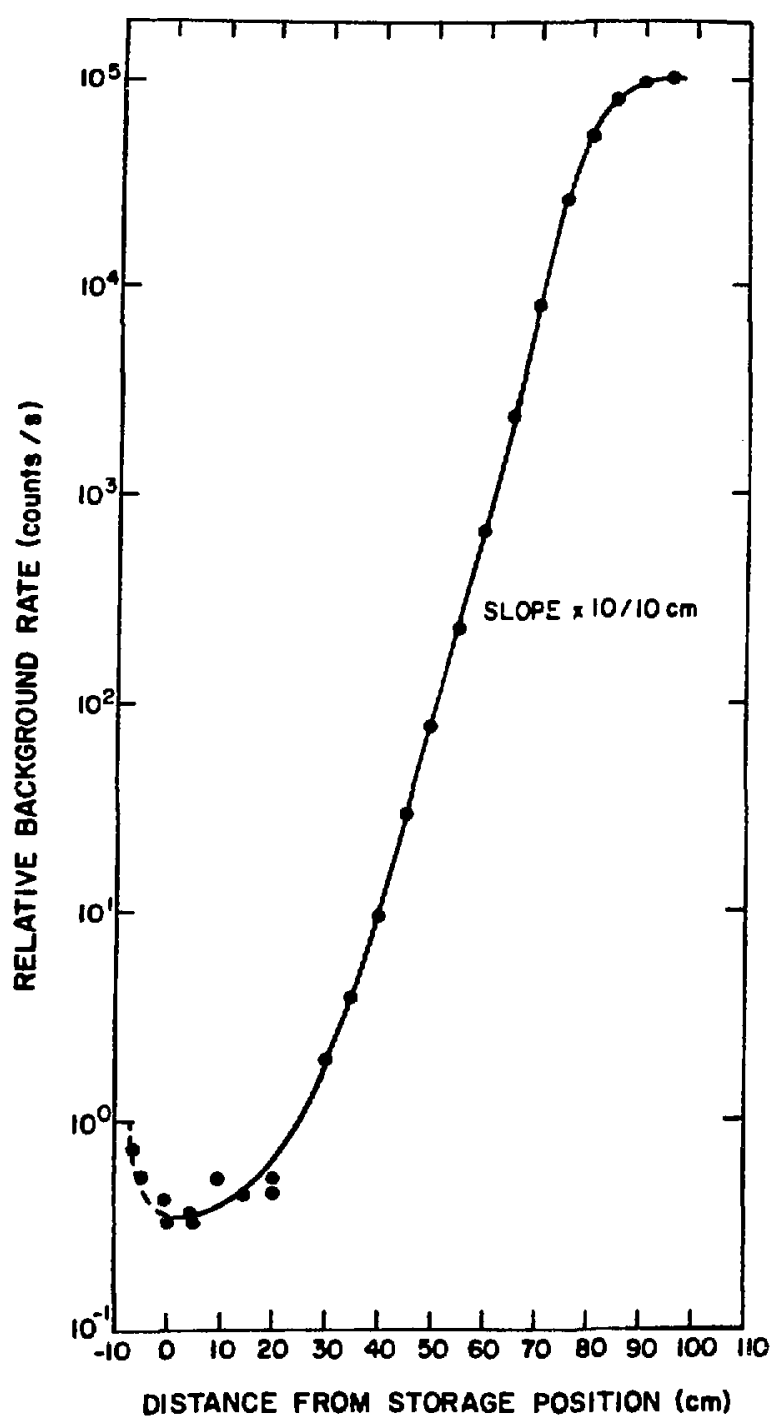

Fig. 57. Relative neutron background rate in detector as a function of distance between source and detector.

a glove port of a glove box (Fig. 58), permitting use of the instrument at different places without contaminating the equipment. Disposable fixtures inside the glovebox can be designed to enable measurements on vials of differing shapes.

Initial evaluation at Los Alamos indicated that, under controlled laboratory conditions, the compact K-edge densitometer could make measurements with uncertainties of $1 \%$. A more advanced instrument was fabricated and delivered to the IAEA for evaluation at the Safeguards Analytical Laboratory in Seibersdorf, Aus-

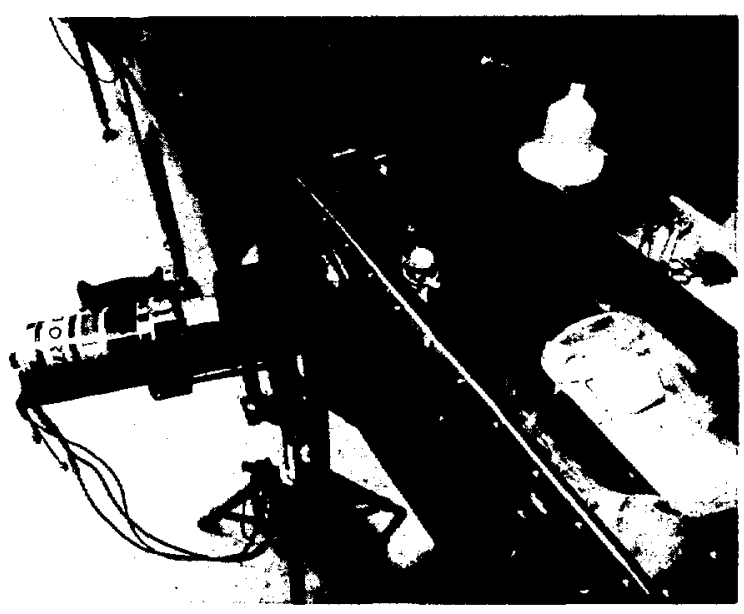

Fig. 58. A view of the compact $\mathrm{K}$-edge densitometer in its measurement position in a glove port. The fixture contaning a Tokaj-style vial is visible just inside the window.

tria. We delivered the instrument in October and trained the IAEA personnel to operate it using plutonium solutions fabricated by the Safeguards Analytical Laboratory. These tests indicated an average mean measurement precision of $0.45 \%$ (RSD). The IAEA has recommended hardware and software refinements that will be addressed in 1984 .

The Joint Research Centre (JRC) in Ispra, Italy, has expressed interest in field testing a similar compact densitometer during the coming year, and other IAEA applications are possible at nuclear facilities in Japan and India. The instrument also could be applied in US plutonium-handling facilities at Rocky Flats, Los Alamos, Richland, or Savannah River.

B. Dounreay Shuffer (G. W. Eccleston, H. O. Menlove, T. R. Van Lyssel, G. Walton, D. C. Garcia, and G. O. Ortiz, Q-1)

We are constructing a delayed-neutron shuffler to measure Prototype Fast Reactor (PFR) leached hulls and centrifuge sludge at the Dounreay Nuclear Power Establishment in Dounreay, Scotland. The shuffler will replace an existing deuterium-tritium neutron generator, ${ }^{54.55}$ and installation of this system will provide the first shuffler measurement of hot scrap and centrifuge sludge in a reprocessing facility.

The present assay system is located in the floor of the PFR fuel-disassembly hot cell and consists of a 250 mm-diam hole surrounded by a 150-mm-thick annulus 
of lead. The lead reduces the gamma dose rate at the detectors to less than $5 \mathrm{R} / \mathrm{h}$. It is surrounded by a 300 mm-thick polyethylene annulus containing nine $\mathrm{BF}_{3}$ detectors arranged in a semicircle. A sealed-tube neutron generator is contained in a port located adjacent to the sample assay hole and provides neutron irradiation of leached hull samples, which represent the majority of material to be assayed. The hulls are placed in a $20-\mathrm{cm}$ diam porous steel basket for measurement and are releached if they contain more than $20 \mathrm{~g}$ of plutonium. Measurements are performed by scanning the basket at five vertical positions.

The shuffler is designed to fit into the hot-cell port to replace the existing neutron generator system and permit the current detectors and sample positioning unit to be used. Electronics for the Dounreay assay system were developed by the Atomic Energy Research Establishment in Harwell, England, but the shufler is operated by a Los Alamos-designed Motorola microprocessor that is being interfaced to the Harwell electronic system. The Harweil electronics and the shuffler unit are shown in Fig. 59 mounted on a pedestal for testing at Los Alamos.

The mechanical design of the shuffler was completed in 1983, and the major mechanical components were manufactured and assembled for testing. The specialized electronics developed by Harwell were shipped by Dounreay personnel to Los Alamos and a PET Commodore computer system was purchased and interfaced with the Harwell electronics. Software development on the PET computer is in progress and scheduled for completion in February 1984 . A ${ }^{252} \mathrm{Cf}$ source will be purchased and shipped to Dounreay in May 1984 for the test and evaluation program. Installation and use of the

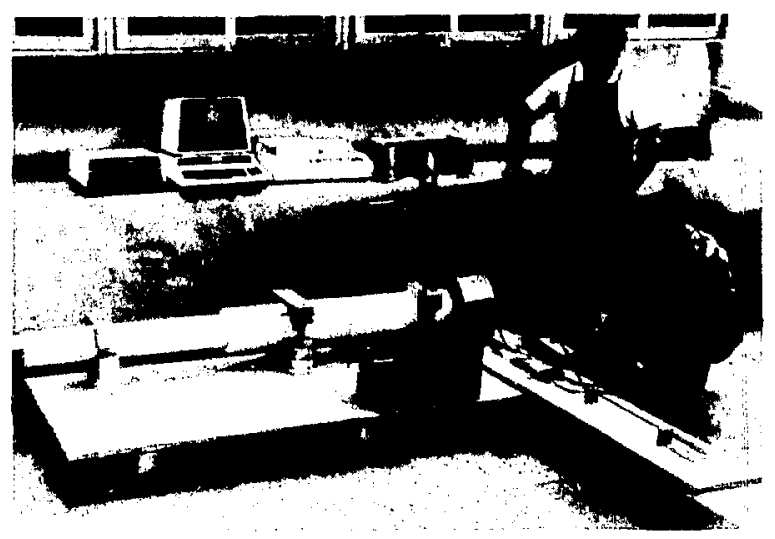

Fig. 59. Shuffler and control electronics mounted on a pedestal for testing at Los Alamos. shuffler by Dounreay personnel are scheduled for July 1984. The results of measurements from reprocessing campaigns will be used for materials accountability.

\section{CONTAINMENT AND SURVEILLANCE FOR INTERNATIONAL SAFEGUARDS}

A. The Interface Between Materials Accounting and Containment/Surveillance (J. T. Markin and R. G. Gutmacher, Q-4; J. P. Shipley, Q-DO/SG)

The IAEA performs inspections of nuclear facilities under the terms of the Treaty on Nonproliferation of Nuclear Weapons to verify that SNM is not diverted for unintended use. These inspections use materials accounting to confirm that facility records of material transfers and inventories are correct and complete and employ containment/surveillance $(\mathrm{C} / \mathrm{S})$ as a complementary measure, primarily to detect anomalies in facility operations. We examined the relationship between these two safeguards measures in a recent report that describes the integration of materials accounting and $\mathrm{C} / \mathrm{S}$ for improved international safeguards.

The report identifies relationships between $\mathrm{C} / \mathrm{S}$ and materials accounting that may be divided into two categories. First, measurement instruments, measurement data, and the material measured should be protected from tampering. Through devices such as seals, tamper resistant enclosures, and surveillance devices, $\mathrm{C} / \mathrm{S}$ provides a secure operating environment for materials accounting. Second, the IAEA must be assured that all significant materials pass through designated key measurement points. Containment/ surveillance provides assurance that key measurement points are not bypassed through a combination of containments that restrict material flows to designated points; surveillance of these points is with film cameras or closed-circuit television. The report summarizes the $\mathrm{C} / \mathrm{S}$ devices that support materials accounting activities and suggests system design principles for integrating materials accounting and $\mathrm{C} / \mathrm{S}$ activities. Examples of materials accounting and $\mathrm{C} / \mathrm{S}$ cooperation in a lightwater reactor and in a reprocessing plant are described.

\section{B. Optimizing the Design of International Safeguards Inspection Systems (J. T. Markin, C. A. Coulter, R. G. Gutmacher, and W. J. Whitty, Q-4)}

Inspections of nuclear facilities by the IAEA and the EURATOM Inspectorate use both materials accounting 
and $\mathrm{C} / \mathrm{S}$ to assure that no nuclear material is diverted for unintended uses. Because international safeguards resources for verifying State compliance with safeguards agreements are limited, an efficient method for allocating inspector verification activities, and their associated technology, is desirable. In effect, design of an inspector's safeguards system must address the tradeoff of resources between activities such as materials accounting and $\mathrm{C} / \mathrm{S}$ to attain the greatest return on the inspectorate resource investment. For example, inspector verification of facility activities may include sampling and measurement of material items, surveillance of storage areas, tamper protection of materials and equipment, and statistical analyses of accounting data. The safeguards design problem may be viewed as selecting the appropriate level of activity or technology for each of these possible inspector activities. However, where there are many potential inspector activities and each activity has several options, the choice of the best system configuration is not obvious. We have developed and reported ${ }^{56}$ a method for selecting a combination of inspector activities that maximizes the likelihood of detecting materials loss or unauthorized facility operations while observing a constraint on safeguards resources.

Our method for selecting an inspector's safeguards system for a facility consists of the following steps:

(1) identify system objectives,

(2) define candidate system designs by specifying fundamental activities that accomplish the objectives,

(3) define the adversary's characteristics and sequences of adversary actions,

(4) develop performance measures that evaluate how weli the system objectives have been accomplished, and

(5) select a most-preferred option from the candidate designs.

For each of these steps, we suggest lists, diagrams, and other tools for organizing the design and evaluation process. These methods are intended to supplement the judgment of the system designer by making explicit the assumptions and value judgments inherent in the design process.

A significant aspect of this design process is the use of optimization theory to select the most preferred design option from the large number of possible combinations of inspection activities. Future work will extend this optimization method from the single-facility application to optimal allocation of inspection resources among multiple States with multiple factlities.

\section{INTERNATIONAL TECHNICAL EX- CHANGES}

For more than a decade, the US safeguards R\&D programs have arranged technical exchanges with similar programs in other countries. Such exchanges improve safeguards technology for all participants. For example, Los Alamos has been active in developing instrumentation such as the Uranium Neutron Coincidence Collar (UNCC), while the EURATOM organization has access to light-water fuel fabrication facilities and reactors. The technical exchange program brings the Los Alamos instruments to operating EURATOM facilities, collecting in-field measurement data, as well as gaining valuable operating experience. Other exchange activities compare two instruments or measurement techniques developed at different laboratories. By comparing results obtained on the same set of materials during the same measurement campaign, instrument developers learn how to improve the accuracy, reliability, and operational ease of their systems. During the past year, Los Alamos has worked with the IAEA, EURATOM (both the Luxembourg inspectorate and the Ispra laboratory), the British Nuclear Materials Accounting and Control Team, the Netherlands ECN, and the Finnish Nuclear Inspectorate.

\section{A. Program of Technical Assistance to IAEA Safe- guards (POTAS) (R. H. Augustson, Q-1)}

The largest exchange activity is conducted with the IAEA and sponsored by POTAS. During 1983 Los Alamos worked on 15 tasks covering instrument development, field exercises, and training. Significant instrument developments included a major upgrade of the HLNCC, development of coincidence counters to measure fast breeder reactor fuel elements and large bottles of plutonium nitrate solution, development of a compact K-edge densitometer, and the commercialization of a portable multichannel analyzer. Twenty-four new inspectors completed the NDA Inspector Training Course at Los Alamos as part of the IAEA's Introductory Course on Agency Safeguards. An additional 20 inspectors were trained in the use of the K-edge densitometer installed at the Tokai reprocessing plant. Two inspection teams (four inspectors each) performed a physical inventory verification at the Hanford Engineering Development Laboratory. This exercise simulated an IAEA inspection as closely as possible but with less time pressure. Under these conditions, the IAEA was 
able to try new approaches and improve its standard procedures.

The IAEA also participated in field exercises organized by EURATOM and Los Alamos. These included measurements of fresh plutonium fuel at Karlsruhe, Federal Republic of Germany (FRG), and spent fuel at two FRG light-water reactors. Figure 60 shows Los Alamos, IAEA, EURATOM, and facility personnel measuring irradiated fuel assemblies with the ION-I electronics package and fork detector at the nuclear power station in Obrigheim, FRG. After these exercises Los Alamos loaned equipment to EURATOM to continue measurements at other facilities; the UNCC loaned to them has been used at 11 European facilities. Equipment has also been loaned to the Netherlands for uranium enrichment measurements and to Finland for measurements of spent fuel.

\section{B. Technical Support to the Power Reactor and Nuclear Fuel Development Corporation (PNC) TASTEX Instru- ments (S.-T. Hsue and P. A. Russo, Q-1)}

As part of the TASTEX program, Los Alamos installed a K-edge densitometer at the $\mathrm{PNC}$ reprocessing plant. We have continued to interact with users of this equipment since the completion of the TASTEX program in 1981, and PNC has expressed interest in minimizing the maintenance overhead involved with this instrument and a plutonium isotopic analyzer provided by LLNL under the TASTEX program. The PNC would like to combine the capabilities of these two instruments into one new assay system. The following summarizes the technical exchanges on this subject that have oc-

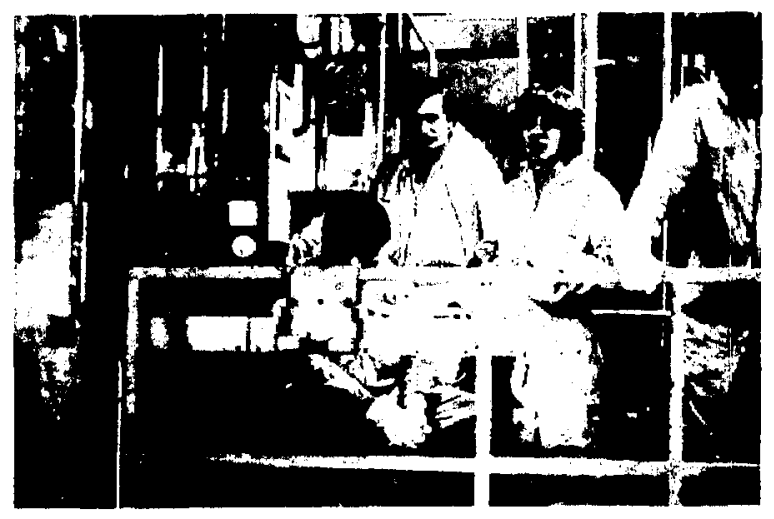

Fig. 60. EURATOM, IAEA, Los Alamos, and facility personnel operating the ION-I and "fork" from the spent-fuel pond bridge at the Obrigheim nuclear power station in the Federal Republic of Germany. curred between Los Alamos and PNC during the past 18 months.

- A meeting of PNC, LLNL, and Los Alamos representatives at Los Alamos in July 1982 produced written recommendations on the technical approach to a combined system. These included retaining separate concentration and isotopics measurement stations with separate analog electronics. A new computer system would be installed to control both measurements simultaneously. The major development effort required for this approach is the software for automation of the assay instruments. A substantial development effort would be required to tailor the isotopics measurement to a new geometry to satisfy a PNC desire to use a single measurement station and data acquisition system. Also, the quality of the isotopics measurement could not be guaranteed, so this additional PNC goal has not been pursued.

- PNC prepared the specifications on a new system for automation of data acquisition and control with multitasking capability. Los Alamos reviewed these specifications and responded with recommendations. PNC ordered the system.

- A meeting at Los Alamos of PNC, LLNL, and Los Alamos representatives was held in September 1983. During this meeting PNC reviewed specific desires for interactive capability, input-output requirements, and access to results for both the concentration and isotopics assay codes. Technical details on hardware requirements also were discussed.

Correspondence with PNC in support of these efforts is still in progress.

\section{Joint Ispra/Los Alamos Program (J. Markin and A. Baker, Q-4)}

1. Data Analysis Program for the IAEA. The JRC at Ispra, Italy, and Los Alamos are engaged in a joint project to provide the IAEA with a computer program for analysis of accounting data from nuclear facilities. This effort, which addresses the need to analyze the increased number of materials balances reported by facilities as they move toward a near-real-time accounting capability, will supply computerized sequential testing procedures that are more appropriate for analysis of large numbers of balances than the currently used fixedlength testing procedures.

Ispra has developed a computer program, ISADAM, that calculates a materials balance sequence and a 
materials balance covariance matrix from basic accounting data. This program will be joined with the Los Alamos program, DECANAL, which contains statistical decision procedures, for use by the Agency in its Statistical Evaluation Section.

\section{Participation in Ispra Course. One Los Alamos} staff member presented an invited lecture at the course, "Nuclear Materials Safeguards: Techniques, Procedures, and Prospects," which was given at the JRC May 24-June 2, 1983. This course addressed the present status of safeguards implementation, including techniques and procedures, and the interface between the operator and the safeguards authority. The Los Alamos lecture compared conventional and near-real-time accounting with emphasis on the verification of these systems by an inspecting agency.

\section{NUCLEAR NONPROLIFERATION ACT (NNPA) COURSE IN NUCLEAR MATERIALS ACCOUNTING (C. R. Hatcher, Q-1)}

The fourth International Training Course on Implementation of State Systems of Accounting for and Control of Nuclear Materials (SSAC) was held October 17. November 4, 1983 in Santa Fe and Los Alamos, New Mexico, and in Richland, Washington. The SSAC courses are given in response to the NNPA of 1978 to provide practical training in the design, implementation, and operation of national systems of nuclear material accounting and control. Los Alamos provides the planning and coordination of these courses, which are sponsored by DOE in cooperation with the IAEA. The Steering Committee and Advisory Group for the 1983 course included representatives of Los Alamos, DOE, IAEA, NRC, US Department of State, Arms Control and Disarmament Agency, and Exxon Nuclear Company, Inc. Nations represented at the course were Australia, Bangladesh, Brazil, Canada, Czechoslovakia, Egypt, France, Japan, Korea, India, Iraq, Malaysia, Pakistan, Philippines, Poland, South Africa, Sweden, Taiwan, and Thailand.

Since their inception in 1980, the SSAC courses have emphasized item facility safeguards in even-numbered years and bulk facility safeguards in odd-numbered years. Emphasis in the 1983 course was on safeguards methods for low-enriched uranium (LEU) conversion and fuel fabrication plants.

The first half of the course, conducted at Santa $\mathrm{Fe}$ and Los Alamos, covered background material, SSAC requirements, and operational safeguards experience at the IAEA, state, and facility levels. Allen Sessoms of the US Department of State spoke on "Development and Current Trends in the International Nuclear Nonproliferation Regime." Other lecturers who provided background information were Carlos Buechler of the IAEA, Mike Smith of the NRC, and Paul Ek of Sweden.

During the 3-week course, one-half of the time was spent in classroom lectures and the other half was spent in workshops, facility tours, and hands-on demonstration: of equipment. The first workshop addressed the IAEA/state systems interface. It began with a session chaired by $O$. B. Johnson of DOE in which each student made a brief presentation concerning nuclear facilities and safeguards implementation in his/her own country. Following these student presentations, questions regarding the IAEA/State systems interface were addressed by a panel of experts, composed of C. Buechler (IAEA), Paul Ek (Sweden), H. Kuroi (Japan), Paul Morrow (NRC), R. Schneider (Exxon Nuclear), and Allen Sessoms (US Department of State, Chairman).

Beginning the second week of the course, students spent 2 days in Los Alamos safeguards laboratories where they used NDA instruments to assay nuclear materials typical of what might be found in a LEU fuel fabrication plant (Fig. 61). Instruments included the neutron coincidence collar for measuring fresh fuel assemblies, the active well neutron coincidence counter for measuring scrap material such as broken pellets, the segmented gamma scanner for measuring low-level waste, and multichannel analyzers with sodium iodide

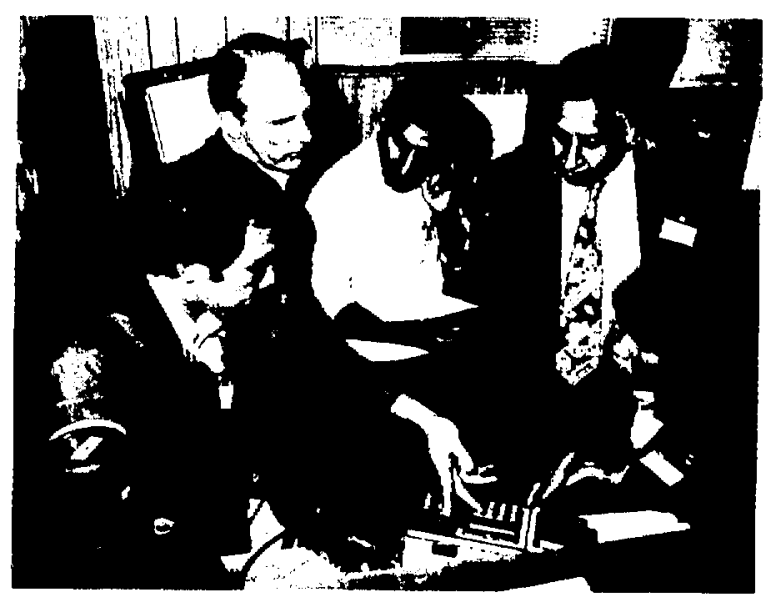

Fig. 61. During the 1983 NNPA training course, Phil Rinard of Los Alamos demonstrated a portable multichannel analyzer to Ahmad Bin Ali of Malaysia, Abdul Nabi of Iraq, Sheikh Mukhtar Ahmed of Pakistan. and Nancy Quey-Ling Lee of Taiwan. 
and germanium detectors for measuring enrichment of $\mathrm{UO}_{2}$ powder in cans.

Because the last half of the course dealt with the practical aspects of implementing safeguards at a LEU fuel fabrication plant, it was conducted at Richland, Washington, where the students had access to a commercial facility operated by Exxon Nuclear Company, Inc. Under contract to Los Alamos, the Exxon Nuclear staff conducted tours of their LEU fuel fabrication plant and provided lectures on the design and implementation of their materials accounting system. John Jaech, from Exxon's corporate office in Bellevue, Washington, described the statistical data analysis procedures associated with bulk processing facility safeguards. While in the Richland area, tours were made to WNP-1 [a Pressurized Water Reactor (PWR) facility that is $60 \%$ complete], the Fast Flux Test Facility . sitor Center operated by Westinghouse, and the NDA equipment at Battelle Pacific Northwest Laboratories.

The final lectures were presented by inspectors familiar with the Exxon plant, Leroy Norderhaug of NRC and Willie Theis of the IAEA. The course concluded with a 2-day workshop in which students were divided into four subgroups. Each subgroup was asked to design a safeguards system for a LEU fuel fabrication plant.

The 1983 SSAC course was longer, had more students (28), and involved more complex logistics than did previous SSAC courses. By holding the lectures in the hotels where the students were staying in Santa Fe and Richland, we encouraged dialogue among students and lecturers. Many of the students commented that they found the informal conversations a key factor in the overall success of the course. The next NNPA course will be presented in 1985 . 


\section{PART 5: RELATED SAFEGUARDS PROJECTS}

In this section, we highlight safeguards work that is related to our DOE/OSS programs but that is sponsored by others.

\section{ESTIMATION METHODS FOR THE PROCESS HOLDUP OF SPECIAL NUCLEAR MATERIALS (K. K. S. Pillay, Q-4, and R. R. Picard, S-1)}

For the past three years, in a research program supported by the NRC, we have been exploring the possibilities of developing statistical estimation models for the holdup of HEU at processing facilities. The program included detailed examination of historical data from processing facilities, nonintrusive in-plant measurements, and specially designed and controlled experiments to develop data and to evaluate the advantages and limitations of predictive models of residual inventories of SNM at processing facilities. We carried out controlled experiments in several processing facilities and used radioactive tracers and special calibration standards to improve the quality of measurements. The major results of this investigation will be used by the $\mathrm{NRC}$, to evaluate objectively the requirements of the proposed MC\&A Reform Amendments, and by the nuclear industry, in developing strategies to comply with these proposed regulations.

- Nondestructive assay of SNM holdup in large equipment and plants is a difficult problem and will remain so because of the inherent limitations of measurement technologies and the layout of plants and equipment.

- Statistical estimation models of holdup in processing equipment and in unit processes can be developed to assist plant operators in meeting reasonable regulatory requirements of holdup estimation as part of periodic inventory development.

- Paramount to developing estimation models is the gathering of good data on holdup as a function of material throughput and other relevant process parameters. After the initial development of holdup data for modeling, occasional updating of models can be done with a limited number of measurements without interfering with plant production schedules.

- To attempt development of holdup estimators for all the equipment in a facility is not prudent because the effort involved in developing estimation models for some equipment cannot justify the benefits derived. A judicious combination of estimation models combined with a limited number of cleanout measurements offers the best approach to developing residual inventories of SNM at processing facilities.

- Most of the historical data on holdup now available at processing facilities are not suitable for the development of predictive models of holdup. Innovative uses of available NDA measurement techniques combined with specially designed calibration standards can contribute significantly to good measurements and better estimation models of SNM holdup in large processing facilities.

These conclusions are ready for a full-scale test at a processing facility to further evaluate the advantages and limitations of statistical estimation methods for developing holdup inventories of SNM.

\section{HANDBOOK OF NUCLEAR SAFEGUARDS MEASUREMENT METHODS (R. G. Gutmacher, Q-4, and C. Thomas, Q-6)}

The Handbook of Nuclear Safeguards Measurement Methods (NUREG/CR-2078) was issued as a hardbound volume in September 1983 by the NRC and the National Technical Information Service. The 733 pages of the handbook contain a critical survey of bulk measurement methods, chemical assay methods, and passive and active NDA methods. Los Alamos contributed the chapter on chemical assay methods.

\section{SECONDARY REFERENCE MATERIALS} FOR THE NRC (J. K. Sprinkle, Jr., R. N. Likes, J. L. Parker, and H. A. Smith, Q-1)

During 1983 we completed a program for the NRC to fabricate calibration reference materials for calibration of HEU measurements made by the safeguards inspectors from the Region II field office in Atlanta. Region Il has a measurement van equipped with several NDA instruments, but lack of calibration standards has forced the inspectors to perform only relative measurements or 
to send samples off site for chemical assay in an attempt to establish absolute reference points. Because this was unsatisfactory, in 1981 we were asked to provide sets of stable reference materials for inspectors' use.

Los Alamos has constructed three sets of reference materials under this program: the first two were mixtures of uranium oxide and graphite, packaged in 2-l plastic bottles, to calibrate gamma-ray assay equipment used to measure high-enriched uranium scrap (Fig. 62), and the third is a series of thin uranium metal foils, enclosed in Petri dishes, to calibrate measurements made on small samples of high-density uranium compounds (Fig. 63). Sets 2 and 3 have been delivered to Region II and should be in use soon. The first set proved unsatisfactory because the physical properties of the powders used prevented complete mixing. Set 2 was fabricated from different feed materials and did mix properly. The experience gained from these efforts is fully documented ${ }^{57,58}$ and should help others to avoid our mistakes. Before shipment to NRC one of the inspectors came to Los Alamos for training in the proper use of the standards with the NRC assay equipment.

Now that fabrication difficulties are understood, future reference materials of these types will probably be

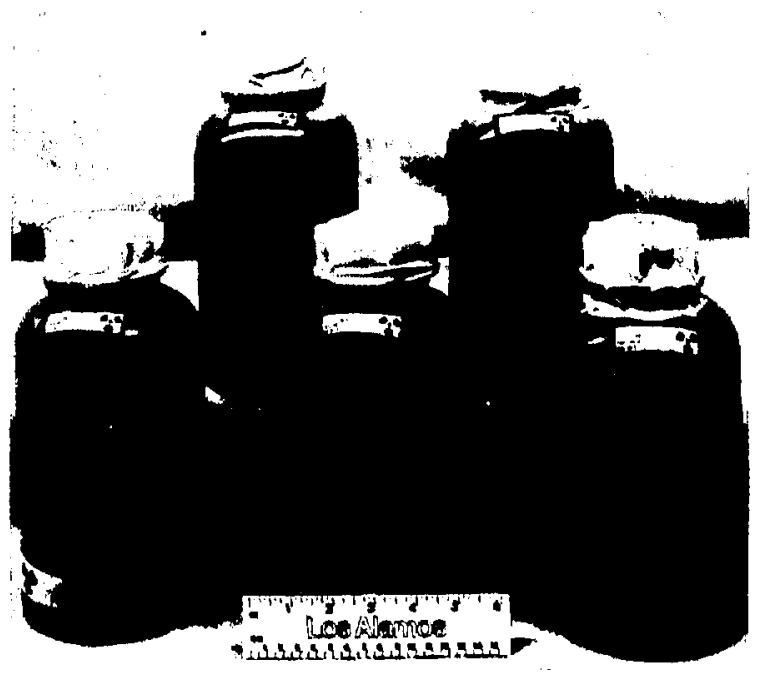

Fig. 62. The uranium oxide plus graphite sel of reference materials. fabricated by standards laboratories such as the DOE's NBL. The experience gained from this project also will be useful for the IAEA support program and several of the large instrumentation projects now under way at Los Alamos.

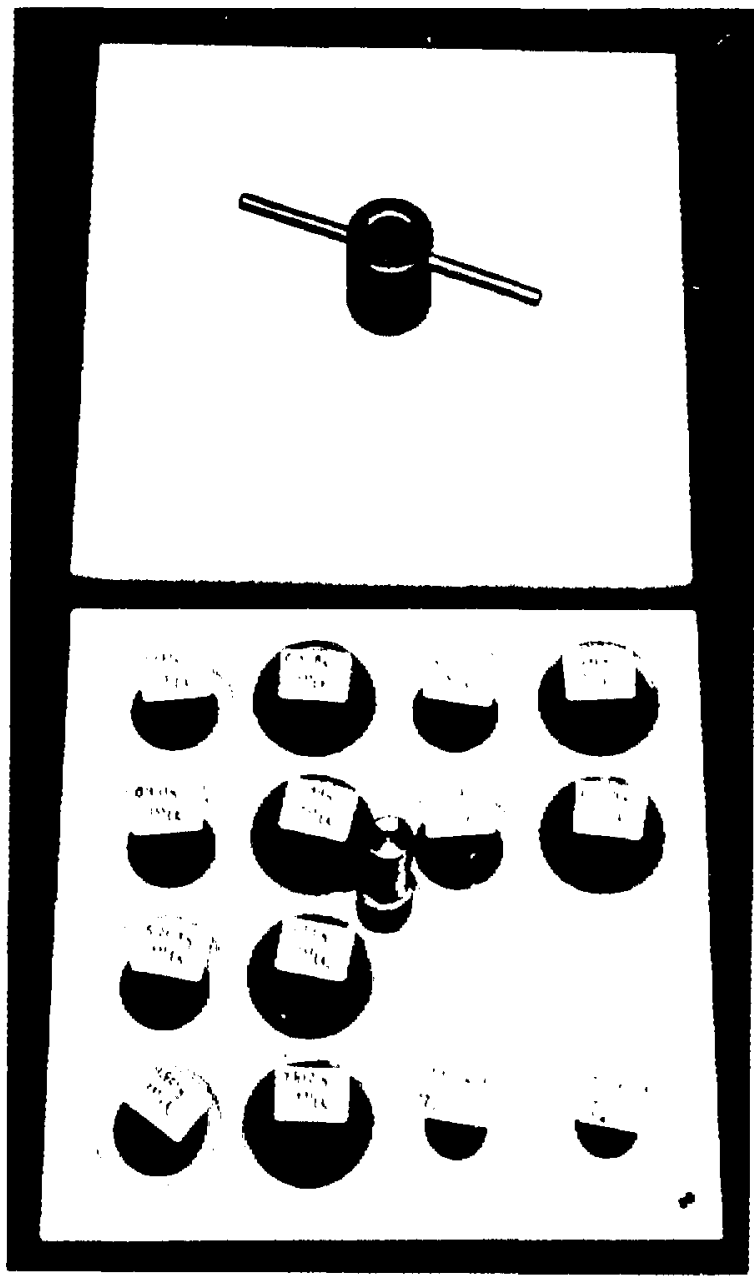

Fig. 63. The thin metal foils of a highly enriched urnnum set of reference materials. 


\section{PUBLICATIONS/PRESENTATIONS}

1. R. H. Augustson, R. B: Walton, W. Harbarger, J. Hicks, G. Timmons, D. Shissler, R. Tayloe, S. Jones, R. Harris, and L. Fields, "Measurements of Uranium Holdup in an Operating Gaseous Diffusion Enrichment Plant," "The ANS/INMM Conference on Safeguards Technology: The ProcessSafeguards Interface," Hilton Head Island, South Carolina, November 28-December 2. 1983 (to be published).

Holdup of nuclear material in process equipment is one of the major sources of uncertainty in materials balances, particularly for high-throughput facilities with large equipment and extensive piping, such as gaseous diffusion uranium-enrichment plants. Locating and measuring the holdup while the plant is operating is a challenging problem because of background from the process material and the neighboring equipment. This paper reports NDA measurements performed at the Goodyear Atomic Gaseous Diffusion Plant, Portsmouth, Ohio, on enrichment equipment at the higher enrichment end $\left(>10 \%{ }^{235} \mathrm{U}\right.$ isotopic abundance) of the cascade. Both neutron and gamma-ray measurements were made to locate anomalously large deposits in converters and compressors and, within the limitations of the techniques, to quantify the amount of the deposit.

2. A. L. Baker, W. C. Fienning, and P. W. Harris, "Computer Safeguards System for the IAEA Inspectors at GCEP," presented at "The ANS/INMM Conference on Safeguards Technology: The Process-Safeguards Interface," Hilton Head Island, South Carolina, November 28-December 2, 1983 (to be published).

The Safeguards Dala System (SDS), which is being developed to provide improved safeguards for the GCEP at Portsmouth, Ohio, is described. The SDS is an automated, integrated computer data acquisition and data management system. It is designed to operate in a continuous unattended mode to provide attributes measurements, summary materials accounting data, and on-sitc data analysis capability to the IAEA inspectors at the GCEP facility.

3. R. C. Bearse and R. M. Tisinger, "Development of a Nuclear Materials Data Base Management System Using PF/LASS Data," Los Alamos National Laboratory, Safeguards Systems Group document Q-4/83-115 (February 1983).

We describe the development of a nuclear materials data base management system using PF/LASS data and a demonstration of the use of that data base in material control and accountability.
(1) We have established that the use of data bases for materials control and accountability can provide significant improvements in programmer productivity while maintaining sufficient speed to support normal plant operations.

(2) We have established, by comparative tests of four data bases and studies of several more, that relational data bases provide the best combination of ease of use, flexibility, and speed.

(3) We have established, by trial and competitive bid, that the system of choice at the present time is INGRES, available 'rom Relational Technology.

(4) We have established a design for the data base and have shown that it provides the flexibility and speed that we will need in a real environmeni.

(5) We have established, by writing dozens of programs, that the INGRES query language (QUEL) and the INGRES-FORTRAN interface (EQUEL) provide about a factor of 10 increase in programmer productivity compared to other available systems.

(6) We have loaded the data base with all Los Alamos Plutonium Facility transactions made between 1 July 1979 and October 1981.

(7) We have developed a package of programs that allows the user to produce MIP and CUSUM graphs (with error-variance propagation) and to generate audit trails for items of interest. Thus, we have established that the data base will allow us to perform calculations and develop reports of potential interest to the operational safeguards group OS-2.

4. R. Beedgen, "Statistical Considerations Concerning Multiple Materials Balance Models," Los Alamos National Laboratory report LA-9645-MS (August 1983).

We explain how an inspector can statistically evaluate collected accounting data within a fixed time to decide if nuclear material has been diverted or not. The fixed time is divided into several accounting periods to enable an early detection of diversion. This report contains the mathematical model for a multiple balance situation. A saddle-point solution for diversion scenario and decision procedure is given only where the detection probability is the point of optimization. A sequential test based on the statistic of cumulative materials balances is presented where a truncation is made at the end of the fixed time. Some ideas are developed on how to quantify the timeliness of a decision. The theoretical considerations are illustrated with the example of a 1000 tonne/yr spent-fuel model reprocessing facility. We show how powerful the considered tests are under an ideal model of a bulk handling plant. 
5. J. T. Caldwell, D. A. Close, T. H. Kuckertz, W. E. Kunz, J. C. Pratt, K. W. Haff, and F. J. Schultz, "Test and Evaluation of A High-Sensivity Assay System for Bulk Transuranic Waste," in "Proceedings of the INMM 24th Annual Meeting on Nuclear Materials Management," Vail, Colorado, July 10-13, 1983, Vol. XII, pp. 75-79.

During the past year, we tested and evaluated the performance of an assay system that accommodates 55-gal. drums of transuranic waste at Oak Ridge $\mathrm{Na}$ tional Laboratory. This neutron assay system provides a routine assay of fissile transuranic isotopes to the l-mg sensitivity level with a pulsed active neutron interrogation based on the differential dieaway technique. A highly sensitive passive neutron measurement determines the content of spontaneous fission transuranic isotopes in each drum as well as an upper-bound estimate of the total alpha activity. All components of the combined, pulsed active and passive neutron assay system performed well on a routine basis during the T\&E period. We performed more than 400 combined passive and active assay measurements of waste drums at Oak Ridge National Laboratory. One-fifth of the initial set of waste drums measured contain less than $100 \mathrm{nCi} / \mathrm{g}$ of total transuranic isotopes and thus qualify legally as nonTRU waste.

6. D. A. Close, J. C. Pratt, J. T. Caldwell, W. E. Kunz, F. J. Schultz, and K. W. Haff, "Multi-Isotopic GammaRay Assay System for Alpha-Contaminated Waste," in "Proceedings of the INMM 24th Annual Meeting on Nuclear Materials Management," Vail, Colorado, July 10-13, 1983, Vol. XII, pp. 70-74.

The capability of an existing segmented gamma-ray system is being expanded to greatly enhance its usefulness. A multienergy gamma-ray scan/transmission measurement is a vital part of any waste assay system that must have the capability of quantifying a diverse list of isotopes within a single drum, including several transuranic species and long-lived fission products. Such a system is being developed for the analysis of alpha-contaminated waste drums at Oak Ridge National Laboratory. A cursory assay of 114 transuranic waste drums of 208- $\ell$ capacity has been made. A more thorough and systematic analysis is now being implemented. Analysis of these data indicates a detection limit better than $100 \mathrm{nCi} / \mathrm{g}$ of waste for the transuranic isotopes ${ }^{237} \mathrm{~Np} /{ }^{233} \mathrm{~Pa},{ }^{239} \mathrm{Pu},{ }^{241} \mathrm{Am}$, and ${ }^{243} \mathrm{Am} /{ }^{239} \mathrm{~Np}$, as well as the long-lived fission products ${ }^{60} \mathrm{Co}$, ${ }^{125} \mathrm{Sb}$, ${ }^{134,137} \mathrm{Cs}$, and ${ }^{154} \mathrm{Eu}$. A pending Code of Federal Regulation (10CFR61) stipulates that the nuclear industry quantify not only its transuranic waste, but aiso certain beta-and gamma-ray-emitting fission products. An assay system based on gamma-ray spectroscopy is the only system that can meet this require- ment for the fission products. Gamma-ray spectroscopy is also required to quantify and identify several transuranic isotopes.

7. T. Crane, "Calibration of a Neutron Coincidence Counter for Measurement of the Plutonium Content of Wet Oxalate Cakes," Los Alamos National Laboratory report LA-9744-MS (April 1983).

A novel calibration procedure has been applied to the dual-ring thermal neutron coincidence counter used at the Los Alamos Plutonium Processing Facility to measure the plutoniumrcontent of wet oxalate cakes. The calibration uses the measured content of the plutonium oxide product and the coincidence counter response to estimate the plutonium content in up to four wet plutonium oxalate cakes. These estimated mass values are then used to calibrate the counter. The calibration calculation is iterative in determining the calibration function coefficients and plutonium masses for oxalate material.

8. H. A. Dayem, A. L. Baker, D. D. Cobb, E. A. Hakkila, and C. A. Ostenak, "Demonstration of Near-Real-Time Accounting: The AGNS 1980-81 Mini-Runs," Los Alamos National Laboratory report LA-9942 (January 1984).

Near-real-time nuclear materials accounting was demonstrated in a series of experiments at AGNS Barnwell Nuclear Fuels Plant. For each experiment, the second and third plutonium cycles were operated continuously for I wk processing uranium solutions. Process data were collected in near-real time by the AGNS computerized nuclear materials control and accounting system and were analyzed for uranium removals using decision analysis techniques developed and implemented by Los Alamos National Laboratory.

Although the measurement system primarily consisted of process-monitoring measurements that were not optimized for near-real-time accounting, the results of uranium removal tests showed that removals and unexpected losses from the process area can be detected. Los Alamos used process-grade measurements to close hourly materials balances. Loss-detection sensitivities for 1 day of between 4 and $18 \mathrm{~kg}$ of uranium, at $50 \%$ detection probability and $2.5 \%$ falsealarm probability, were calculated for selected accounting areas. Using pulsed-column inventory estimators, we calculated a total four-column inventory that was within $10 \%$ of column dump measurements. Loss-detection sensitivity could be improved by incorporating on-line waste stream measurements, improving laboratory measurements for process streams, and refining the pulsed-column inventory estimates. 
9. H. A. Dayem, E. A. Hakkila, A. L. Baker, and D. D. Cobb, "Materials Loss-Detection Sensitivities Using Process Grade Measurements at AGNS BFNP," presented at "The ANS/INMM Conference on Safeguards Technology: The Process-Safeguards Interface," Hilton Head Island, South Carolina, November 28-December 2, 1983 (to be published).

Process quality measurement data from cold runs at AGNS BNFP are used to demonstrate near-real-time accounting by closing hourly materials balances and to evaluate contactor inventory estimation techniques. Loss-detection sensitivities for 1 day of between 4 and $18 \mathrm{~kg}$ uranium, at $50 \%$ detection probability and $2.5 \%$ false-alarm probability, are calculated for selected accounting areas. Pulsed-column inventory estimators are used to calculate an inventory that is generally within $10 \%$ of column dump measurements. Loss-detection sensitivity could be improved by incorporating on-line waste stream measurements, improving laboratory measurements for process streams, and refining the pulsed-column inventory estimates.

10. G. W. Eccleston, "Analytical Applications for Delayed Neutrons," invited paper presented at "Specialists Meeting on Yields and Decay Data of Fission Product Nuclides," Upton, New York, October 24-27, 1983.

Analytical formulations that describe the time dependence of neutron populations in nuclear materials contain delayed-neutron dependent terms. These terms are important because the delayed neutrons, even though their yields in fission are small, permit control of the fission chain reaction process. Analytical applications that use delayed neutrons range from simple problems that can be solved with the point reactor kinetics equations to complex problems that can only be solved with large codes that couple fluid calculations with the neutron dynamics. Reactor safety codes, such as SIMMER, model transients of the entire reactor core using coupled spacetime neutronics and comprehensive thermal-fluid dynamics. Nondestructive delayed-neutron assay instruments are designed and modeled using a threedimensional continuous-energy Monte Carlo code. Calculations on high-burnup spent fuels and other materials that contain a mix of uranium and plutonium isotopes require accurate and complete information on the delayed-neutron periods, yields, and energy spectra. A continuing need exists for delayedneutron parameters for all the fissioning isotopes.

11. G. W. Eccleston, S. S. Johnson, H. O. Menlove, T. Van Lyssel, D. Black, B. Carlson, L. Decker, and M. W. Echo, "FAST Facility Spent-Fuel and Waste Assay Instrument," presented at "The ANS/INMM Conference on Safeguards Technology: The Pro-
cess-Safeguards Interface," Hilton Head Island, South Carolina, November 28-December 2, 1983 (to be published).

A delayed-neutron assay instrument was installed in the Fluorinel Dissolution and Fuel Storage Facility at INEL. The dual-assay instrument is designed to measure both spent fuel and waste solids that are produced from fuel processing. A set of waste standards, fabricated by Los Alamos using uranium supplied by EXXON Nuclear Idaho Company, was used to calibrate the small-sample assay region of the instrument. Performance testing was completed before installation of the instrument to determine the effects of uranium enrichment, hydrogenous materials, and neutron poisons on assays. The unit was designed to measure high-enriched uranium samples in the presence of large neutron backgrounds. Measurements indicate that the system can assay low-enriched uranium samples with moderate backgrounds if calibrated with proper standards.

12. M. L. Evans, "Calculation of Terrestrial GammaRay Fields in Airborne Radiometric Surveys," Los Alamos National Laboratory report LA-9741-MS (July 1983).

Terrestrial gamma-ray fields have been calculated for points in air above semi-infinite rock formations containing known concentrations of the naturally occurring radionuclides ${ }^{40} \mathrm{~K},{ }^{235} \mathrm{U},{ }^{238} \mathrm{U},{ }^{232} \mathrm{Th}$, and their daughter products. Energy and angle-dependent gamma-ray fluxes were computed using a discrete ordinates transport code. The density and composition of the rock medium as well as the air density and survey height were varied to determine their effect on the observed gamma-ray flux spectrum. Variations in formation porosity or water saturation cause little spectral shape perturbation above $200 \mathrm{keV}$ and result chiefly in a scalar change that is related to the mass-density-weighted average Z/A of the rock formation. Corrections to the flux spectra for variations in air density and surface elevation can be made by simple scaling with density/altitude. However, the spectral shape of the observed gamma-ray flux depends strongly on the survey height above the rock/air interface, so that spectral stripping parameters must be determined as functions of survey height.

13. P. E. Fehlau, "Gamma-Ray Detectors for Intelligent, Hand-Held Radiation Monitors," in "Proceedings of the 1983 IEEE Transactions on Nuclear Science," San Francisco, California, October 19-21, 1983, Vol. NS-31, No. 1, pp. 664-671. Small radiation detectors based on $\mathrm{Hgl}_{2}$, bismuth germanate (BGO), plastic, or $\mathrm{NaI}(\mathrm{Tl}$ ) detector materials were evaluated for use in small, lightweight radiation monitors. The two denser materials, $\mathrm{Hgl}_{2}$ and $\mathrm{BGO}$, had poor resolution at low energy and thus 
performed less well than $\mathrm{NaI}(\mathrm{Tl})$ in detecting lowenergy gamma rays from bare, enriched uranium. The plastic scintillator, a Compton recoil detector, also performed less well at low gamma-ray energy. Two small $\mathrm{Nal}(\mathrm{TI})$ detectors were suitable for detecting bare uranium and shieli.ud plutonium. One became part of a new lightweight hand-held monitor and the other found use as a pole-mounted detector for monitoring hard-to-reach locations.

14. P. E. Fehlau, "Vehicle SNM Monitors: Results of an Evaluation," in "The Proceedings of the INMM 24th Annual Meeting on Nuclear Materials Management," Vail, Colorado, July 10-13, 1983, Vol. XII, pp. 294-300.

Several types of radiation monitors, designed to search motor vehicles for special nuclear materials at area exit gates, were constructed and evaluated at the Los Alamos National Laboratory. These monitors consist of stationary radiation detection equipment or portable, hand-held instruments. The stationary monitors have either a detector portal through which vehicles pass or an underground detector array to measure radiation intensity from below a parked vehicle. Portal monitors straddle a vehicle path where they monitor vehicles in motion (at an ideal speed of 8 $\mathrm{km} / \mathrm{h}$ ) as they approach the exit gate, whereas the underground detector array is located in the roadbed at an exit station where vehicles stop for inspection. Roadbed monitors require a monitoring period of about one minute; hand-held monitors require several minutes. The sensitivity of the monitors depends on the size of the vehicle being monitored, the position and type of the nuclear material inside the vehicle, and the type and position of the radiation detectors. For small vehicles, adequate sensitivity can be obtained with any of the monitors. For slow-moving vehicles up to $3.4 \mathrm{~m}$ high, the vehicle portal offers lowcost performance. The best performance, however, at a higher installation cost, is offered by the roadbed monitor supplemented with overhead detectors.

15. P. E. Fehlau, "Quantitative Radiation Monitors for Containment and Surveillance," in "The Proceedings of the ESARDA Fifth Annual Symposium on Safeguards and Nuclear Materials Management," Versailles, France, April 19-21, 1983, pp. 153-157.

Quantitative radiation monitors make it possible to differentiate between shielded and unshielded nuclear materials. The hardness of the gamma-ray spectrum is the attribute that characterizes bare or shielded material. Separate ligh- and low-energy gamma-ray regions are obtained from a single-channel analyzer through its window and discriminator outputs. The monitor counts both outputs and computes a ratio of the highand low-energy region counts whenever an alarm occurs. The ratio clearly differentiates between shielded and unshielded nuclear material so that the net alarm count may be identified with a small quantity of unshielded material or a large quantity of shielded material. Knowledge of the diverted quantity helps determine whether an inventory should be called to identify the loss.

16. P. E. Fehlau, C. Garcia, Jr., R. A. Payne, and E. R. Shunk, "Vehicle Monitors for Domestic Perimeter Safeguards," Los Alamos National Laboratory report LA-9633-MS (January 1983).

This report compares several types of sperial nuclear material monitors used to search motor ' ' 'nicles at area exit gates. These monitors use either portable, hand-held instruments or stationary radiation detection instruments. Stationary monitors have a detector portal or a detector array located in the roadbed at a vehicle exit. Static measurements and operational evaluation provide a performance comparison of three types of monitors: hand-held instruments, vehicle portals, and a high-sensitivity roadbed monitor. Still higher sensitivity is possible by placing detectors overhead as well as in the roadbed; such a combined monitor does not yet exist, but the report estimates its performance. The cost for a combined monitor increases significantly when both a canopy and a roadbed detector pit are constructed; however, the performance of the combined monitor exceeds that obtained with other monitor configurations.

17. P. E. Fehlau, J. C. Pratt, J. T. Markin, and T. Scurry, Jr., "Smarter Radiation Monitors for Safeguards and Security," in "The Proceedings of the INMM 24th Annual Meeting on Nuclear Materials Management," Vail, Colorado, July 10-13, 1983, Vol. XII, pp. 122-128.

Radiation monitors for nuclear safeguards and security depend on internal control circuits to determine when diversion of special nuclear materials is taking place. Early monitors depended on analog circuits for this purpose; subsequently, digital logic controllers made better monitoring methods possible. Now, versatile microprocessor systems permit new, more efficient, and more useful monitoring methods. One such method is simple stepwise monitoring, which has variable aiarm levels to expedite monitoring where extended monitoring periods are required. Another method, sequential probability ratio logic, tests data as it accumulates against two hypotheses-background, or background plus a transient diversion signal-and terminates monitoring as soon as a decision can be made that meets false-alarm and detection confidence requirements. A third method, quantitative monitoring for personnel, calculates count ratios of high- to low-energy gamma-ray regions to predict whether the material detected is a small quantity of bare material or a larger quantity of shielded material. In addition, microprocessor system subprograms can assist in detector calibration and trouble-shooting. Examples of 
subprograms are a variance analysis technique to set bias levels in plastic scintillators and a state-of-health routine for detecting malfunctions in digital circuit components.

18. W. Ford, "How to Talk to Your Computer-Literally," presented at "The 1983 Fall DECUS US Symposium," Las Vegas, Nevada, October 24-28, 1983.

Voice $1 / O$ is a rapidly growing technology. The techniques are still in the formative stage and thus are still somewhat primitive. However, useful tasks can be accomplished within the limitations of today's hardware. No reference to specific hardware will be given, but guidelines for selecting and using hardware will be provided. The guidelines include vocabulary. size, method of training, upload/sownload capabilities, user control of recognition parameters, package formfactor, and information returned to the user. Hints will be given for working with hardware limitations. Some current and proposed uses for voice $\mathrm{I} / \mathrm{O}$ at Los Alamos National Laboratory will be described. These applications include personnel identity verification, instrument control, data entry, alarm annunciation, and issuing operating system and editor commands.

19. A. S. Goldman, G. L. Anderson, and J. Weber, "Cumulative Error Models for the Tank Calibration Problem," in "The Proceedings of the INMM 24th Annual Meeting of Nuclear Materials Management," Vail, Colorado, July 10-13, 1983, Vol. XII, pp. 187-194.

The purpose of a tank calibration equation is to obtain an estimate of the liquid volume that corresponds to a liquid level measurement. Calibration experimental errors occur in both liquid level and liquid volume measurements. If one of the errors is relatively small, the calibration equation can be determined from wellknown regression and calibration methods. If both variables are assumed to be in error, then for linear cases a prototype model should be considered. Many investigators are not familiar with this model or do not have computing facilities capable of obtaining numerical solutions. This paper discusses and compare; three linear models that approximate the prototype model and have the advantage of much simpler computations. Comparisons among the four models and recommendations of suitability are made from simulations and from analyses of six sets of experimental data.

20. A. S. Goldman, "Fitting a Straight Line to Cumulative Data When Both Variables Are Subject to Error," presented at the 1983 Joint Statistical Meetings, Toronto, Canada, August 15-18, 1983.

Consider the model in which $X$ and $Y$ are related by $Y=\alpha+\beta \Sigma_{-j} X_{j}$, where $\alpha$ and $\beta$ are unknown, and we observe $y=Y+d$ and $x_{j}=X_{j}+e_{j}$, where $d$ and $e_{j}$ are random variables. We survey the procedures for estimating $\alpha$ and by considering various forms of the distributions of the errors ( $d$ and e). Applications are given for tank calibration data that previously have been analyzed under the assumption that either $\mathrm{d}$ or $\mathrm{e}$ is identically zero.

21. A. S. Goldman, "Improved Attribute Sampling Plans for Materials Accounting," Los Alamos National Laboratory report LA-9935-MS (November 1983).

The Safeguards Systems Group at the Los Alamos National Laboratory has been requested to study attribute sampling plans requiring minimal sample sizes for the purpose of reducing personnel time and radiation exposure in item inventories of nuclear materials. Results indicate that double sampling plans can generally reduce the work effort by at least $30 \%$ compared with the single sampling plans currently used at the Los Alamos plutonium facility. Such innovations should be applicable to any DOE facility engaged in processing nuclear materials, particularly in low-turnover storage areas. Future work includes the study of multiple sampling plans as a possible procedure for further reduction of sample sizes.

22. A. S. Goldman and R. S. Marshall, "Fine Tuning of a Measurement Control Program at Los Alamos National Laboratory," presented at "The ANS/INMM Conference on Safeguards Technology: The Process-Safeguards Interface," Hilton Head Island, South Carolina, November 28-December 2, 1983 (to be published).

This paper suggests a revised measurement control program (MCP) for balances at the Los Alamos National Laboratory plutonium facility. The revised MCP is based on an analysis of data taken from June 1981 through August 1983. The most important finding in our study is that significant measurement bias occurs in nearly every balance. An important cause of this bias has been traced to truncation errors, and a detailed discussion of the effects of truncation errors is presented. We also discuss other sources of bias and their resolution, and finally, we suggest methods for determining accuracy, precision, and randomness of measurements of weights and the response to failures of statistical tests.

23. R. G. Gutmacher, E. A. Kern, J. F. Hafer, M. J. Roybal, and S. T. Smith, "Modeling and Simulation of the Secure Automated Fabrication Line Boat Transport System," Los Alamos National Laboratory, Safeguards Systems document Q-4/83-423 (July 1983).

Computer modeling and simulation of the Boat Transport System in the Secure Automated Fabrication line is discussed. The operating times of Boat Inspection and Cleaning and the handling of empty 
boats were found to be crucial factors. Of the three scenarios investigated, two gave satisfactory results. One scenario is suitable if all boats must be inspected and cleaned before re-use. The other scenario is preferable if empty boats can be re-used without being inspected. In both of the satisfactory scenarios the conveyor loops are never more than $45 \%$ filled and are in operation less than $30 \%$ of the available time.

24. R. G. Gutmacher, E. A. Kern, D. R. Duncan, and M. W. Benecke, "Modeling and Simulation for Process and Safeguards System Design," presented at "The ANS/INMM Conference on Safeguards Technology: The Process-Safeguards Interface," Hilton Head Island, South Carolina, November 28December 2, 1983 (to be published).

A computer modeling and simulation approach that meets the needs of both the process and safeguards system designers is described. The results have been useful to Westinghouse Hanford Company process designers in optimizing the process scenario and operating scheme of the Secure Automated Fabrication line. The combined process/measurements model will serve as the basis for design of the safeguards system. Integration of the process design and the safeguards system design should result in a smoothly operating process that is easier to safeguard.

25. R. G. Gutmacher and C. C. Thomas, "Chemical Assay Methods," in Handbook of Nuclear Safeguards Measurements Methods, D. R. Rogers, Ed. (Nuclear Regulatory Commission, Washington, DC, and National Technical Information Service, Springfield, Virginia, September 1983), NUREG/CR-2078, Chap. IV.

Abstract unavailable.

26. H. H. Hsu, E. J. Dowdy, G. P. Estes, M. C. Lucas, J. M. Mack, C. E. Moss, and M. E. Hamm, "Efficiency of a Bismuth-Germanate Scintillator: Comparison of Monte Carlo Calculations With Measurements," in "The Proceedings of the 1983 IEEE Nuclear Science Symposium," San Francisco, California, October 19-21, I 983, Vol. NS31, No. 1.

Monte Carlo calculations of a bismuth-germanate scintillator's efficiency agree closely with experimental measurements. For this comparison, we studied the absolute gamma-ray photopeak efficiency of a scintillator ( $7.62 \mathrm{~cm}$ long by $7.62 \mathrm{~cm}$ in diameter) at several gamma-ray energies from 166 to $2615 \mathrm{keV}$ at distances from 0.6 to $152.4 \mathrm{~cm}$. Computer calculations were done in a two-dimensional cylindrical geometry with the Monte Carle coupled photon-electron code CYLTRAN. For the experiment we measured 11 sources with simple spectra and precisely known strengths. The average deviation between the calculations and the measurements is $3 \%$. Our calcu- lated results also closely agree with recently published calculated results.

27. S. -T. Hsue and T. Marks, "Measurement Control Program for NDA Instruments," in "The ANS/INMM Conference on Safeguards Technology: The Process-Safeguards Interface," Hilton Head Island, South Carolina, November 28-December 2, 1983 (to be published).

Measurement control checks for nondestructive assay instruments have been a constant and continuing concern at Los Alamos National Laboratory. This paper summarizes the evolution of the measurement control checks in the various high-resolution gamma systems we have developed. In-plant experiences with these systems and checks will be discussed. Based on these experiences, a set of measurement control checks is recommended for high--esolution gammaray systems.

28. S. -T. Hsue, S. F. Marsh, and T. Marks, "Hybrid Chemical and Nondestructive Analysis Technique," in "The Proceedings of the ESARDA Fifth Symposium on Safeguards and Nuclear Material Management," Versailles, France, April 19-2I, I983, pp. 209-212.

A hybrid chemical/NDA technique has been applied at the Los Alamos National Laboratory to the assay of plutonium in ion-exchange effluents. Typical effluent solutions contain low concentrations of plutonium and high concentrations of americium. A simple trioctylphosphine oxide (TOPO) separation can remove $99.9 \%$ of the americium. The organic phase that contains the separated plutonium can be accurately assayed by monitoring the uranium $\mathrm{L} x$-ray intensities.

29. K. J. Johnson, "Operation Manual: MiniSegmenied Gamma Scanner," Los Alamos National Laboratory report LA-9350-M (June 1983).

An operation and maintenance manual for a microcomputer-controlled segmented gamma scan table is presented. It has a high step and position resolution and a high degree of mechanical and electronic stability. The specifications are presented along with operating instructions, circuits, and software.

30. K. Johnson, "A Microcomputer-Based Stepping Motor Controller," Los Alamos Nitional Laboratory report LA-9731-MS (April 1983)

A microcomputer-controlled stepping motor is described. A Motorola MC68701 microcomputer unit is interfaced to a Cybernetic CY500 stored-program controller that outputs through Motorola input/output isolation modules to the stepping motor. A complex multifunction controller with enhanced capabilities is thus available with a minimum number of parts. 
31. E. A. Kern, "Systems Modeling and Performance Measures Study: Phase I Final Report," Los Alamos National Laboratory, Safeguards Systems Group preliminary document Q-4/83-272.

This report summarizes the activities under Phase I of the Systems Modeling and Performance Measures Study sponsored by the Transuranic Waste Systems Office, Rocky Flats Plant. The overall objective of the study is to determine the usefulness of simulation modeling in designing the Transuranic Waste Management System. In this Phase I Report (I) the transuranic waste system network is defined, (2) the systems performance measures are discussed, (3) simulation and analytical modeling are compared, (4) the preliminary simulaxion model is described, (5) input data requirements are defined, and (6) the feasibility of transferring the model to the Rocky Flats Plant is discussed.

32. M. S. Krick and J. E. Swansen, "Neutron Multiplicity and Multiplication Measurements," Los Alamos National Laboratory document LAUR-83-2123 (accepted for publication in NIM journal).

A neutron multiplicity analysis system has been developed to determine the neutron multiplication of nonmoderating plutonium samples measured with thermal neutron counters and shift-register coincidence electronics. The system also determines the $(\alpha, n)$ neutron source strength and the effective ${ }^{240} \mathrm{Pu}$ mass of the samples. The complete system is described and the measurement results are presented. Typical mass errors are $\sim 2 \%(l \sigma)$. The principal limitation of the system is the long measurement time required for a samiple with high plutonium mass or' large $(\alpha, n)$ component.

3. T. K. Li, T. E. Sampson, and S. S. Johnson, "Plutonium Isotopic Measurement for Small Product Samples," in "The Proceedings of the ESARDA Fifth Symposium on Safeguards and Nuclear Material Management," Versailles, France, April 19-21, 1983, pp. 289-291.

An automated at-line plutonium isotopic analysis system for small product plutonium samples in the range of 10 to $1100 \mu \mathrm{g}$ has been developed. The analysis is based on low-energy gamma rays at $43.48 \mathrm{keV}\left({ }^{238} \mathrm{Pu}\right)$, $45.23 \mathrm{keV}\left({ }^{240} \mathrm{Pu}\right), 51.63 \mathrm{keV}\left({ }^{239} \mathrm{Pu}\right), 64.83 \mathrm{keV}$ $\left.{ }^{24}\left(\mathrm{Pu}^{237} \mathrm{U}\right), 129.3 \mathrm{keV}{ }^{239} \mathrm{Pu}\right)$, and $148.6 \mathrm{keV}\left({ }^{241} \mathrm{Pu}\right)$. Within a 20-ks counting time, we demonstrated that for plutonium masses $>600 \mu \mathrm{g}$ the precision is $<0.7 \%$ for ${ }^{238} \mathrm{Pu} /{ }^{239} \mathrm{Pu}$ and ${ }^{240} \mathrm{Pu} /{ }^{239} \mathrm{Pu}$ ratios and $\sim 4 \%$ for the ${ }^{241} \mathrm{Pu} /{ }^{239} \mathrm{Pu}$ ratio. The agreement between the results measured by gamma-ray spectroscopy and those measured by mass spectrometry for seven samples is within the gamma-ray precision limits.
34. T. K. Li, "Determination of Plutonium Isotopic Ratios by Using Low-Energy Gamma-Ray Spectroscopy," in "The ANS/INMM Conference on Safeguards Technology: The Process-Safeguards Interface," Hilton Head Island, South Carolina, November 28-December 2, 1983 (to be published).

A nondestructive gamma-ray technique has been developed to determine plutonium isotopic ratios. The technique is based on the high-intensity, low-energy gamma rays at $43.48,45.23,51.63,59.54$, and 64.83 keV for ${ }^{238} \mathrm{Pu},{ }^{240} \mathrm{Pu},{ }^{239} \mathrm{Pu},{ }^{241} \mathrm{Am}$, and ${ }^{241} \mathrm{Pu}$, respectively. The results demonstrate that this technique can accurately measure plutonium samples in a timely manner and in a wide range of masses, isotopic contents, chemical forms, and ages from chemical processing.

35. T. K. Li, T. Marks, and J. L. Parker, "Solution Assay Instrument Operations Manual," Los Alamos National Laboratory report LA-9820-M (September 1983).

An at-line solution assay instrument (SAI) has been developed at the Los Alamos National Laboratory and installed in a plutonium purification and americium recovery process area in the Los Alamos Plutonium Processing Facility. The instrument was designed for accurate, timely, and simultaneous nondestructive analysis of plutonium and americium in process solutions that have a wide range of concentrations and americium/plutonium ratios, and for routine operation by process technicians who lack instrumentation background. The SAI, based on transmission-corrected, high-resnlution gamma-ray spectroscopy, has two measurement stations attached to a single multichannel analyzer/computer system. To ensure the quality of assay results, the SAl has an internal measurement control program, which requires daily and weekly check runs and monitors key aspects of all assay runs. For a 25 -me sample, the assay precision is $<1 \%$, both for plutonium and for americium having concentrations $>5 \mathrm{~g} / \mathrm{\ell}$ within a 2000-s count time.

36. J. T. Markin, C. A. Coulter, R. G. Gutmacher, and W. J. Whitty, "Optimizing the Design of International Safeguards Inspection Systems," in "The Proceedings of the ESARDA Fifth Annual Symposium on Safeguards and Nuclear Material Management," Versailles, France, April 19-21, 1983, pp. 5I-55.

Efficient implementation of international inspections for verifying the operation of a nuclear facility requires that available resources be allocated among inspection activities to maximize detection of misoperation. This report describes a design and evaluation method for selecting an inspection system that is 
optimal for accomplishing inspection objectives. The discussion includes methods for identifying system objectives, defining performance measures, and choosing between candidate systems. Optimization theory is applied in selecting the most preferred inspection design for a single nuclear facility, and an extension to optimal allocation of inspection resources among States containing multiple facilities is outlined.

37. J. T. Markin, C. A. Coulter, R. G. Gutmacher, C. C. Thomas, Jr., and W. J. Whitty, "Design and Evaluation Methods for an Integrated Safeguards System," Los Alamos National Laboratory report LA-9964, Vols. I and II (March 1984).

An integrated safeguards system is defined as a collection of safeguards activities in which system components are coordinated to accomplish safeguards objectives efficiently within constraints imposed by safeguards resources, facility operations, potential adversaries, and regulatory requirements. This report describes a method for designing and evaluating an integrated safeguards system that consists of four parts: (1) a problem definition phase that specifies resources and consiraints comprising the problem boundary values, (2) a system synthesis phase that describes how to select and integrate safeguards activities for efficient attainment of system objectives, (3) a system analysis/optimization phase that defines measures of safeguards performance and develops methods for evaluating them, and (4) a decisionmaking phase that develops principles for selecting admissible designs and preference ordering designs.

38. J. T. Markin and R. G. Gutmacher, "The Interface Between Materials Accounting and Containment/Surveillance," Los Alamos National Laboratory report LA-9976-MS (December 1983).

Inspections of nuclear facilities by the International Atomic Energy Agency to verify compliance with the terms of the Nuclear Nonproliferation Treaty combine both materials accounting (NA) and containment/surveillance $(\mathrm{C} / \mathrm{S})$ methods. Interfaces between these two safeguards measures are necessary to assure that the operator's and inspector's measurements of material are accurate and complete. This report describes those interfaces where $\mathrm{C} / \mathrm{S}$ can provide the necessary protection for measurement instruments and information used in MA. General considerations that influence the $\mathrm{MA}$ and $\mathrm{C} / \mathrm{S}$ interface are described. The report also includes specific examples of interactions between materials accounting and $\mathrm{C} / \mathrm{S}$ in a lightwater reactor and in a reprocessing facility.

39. J. T. Markin and J. P. Shipley, "A Comparison of Conventional and Near-Real-Time Accounting for International Safeguards," Los Alamos National Laboratory document LA-UR-83-1455, presented as a lecture at the "Nuclear Materials Safeguards Techniques, Procedures and Prospects Course," Ispra, Italy, May 24-June 2, 1983.

No abstract available.

40. H. O. Menlove, "Standardization of Portable Assay Instrumentation-The Neutron Coincidence Tree," in "The Proceedings of the ESARDA Fifth Symposium on Safeguards and Nuclear Material Management," Versailles, France, April 19-21, 1983, pp. 231-240.

Standardization of portable neutron assay instrumentation has been achieved by using the neutron coincidence technique as a common basis for a wide range of instruments and applications. The electronics originally developed for the High-Level Neutron Coincidence Counter has been adapted to both passiveand active-assay instrumentation for field verification of bulk plutonium, inventory samples, pellets, powders, nitrates, high-enriched uranium, and materials-testing-reactor, light-water-reactor, and mixed-oxide fuel assemblies. The family of detectors developed at Los Alamos National Laboratory and their performance under in-field conditions are described.

41. C. E. Moss, W. Bernard, E. J. Dowdy, C. Garcia, M. C. Lucas, and J. C. Pratt, "A Gamma-Ray Spectrometer System with High Efficiency and High Resolution," in "The Proceedings of the ESARDA Fifth Symposium on Safeguards and Nuclear Materials Management," Versailles, France, April 19-21, 1983, pp. 293-295.

Our gamma-ray spectrometer system, designed for field use, offers high efficiency and high resolution for safeguards applications. The system consists of three $40 \%$ high-purity germanium detectors and a LeCroy 3500 data acquisition system that calculates a composite spectrum for the three detectors. The LeCroy 3500 mainframe can be operated remotely from the detector array with control exercised through modems and the telephone system. System performance with a mixed source of ${ }^{125} \mathrm{Sb},{ }^{154} \mathrm{Eu}$, and ${ }^{155} \mathrm{Eu}$ confirms the expected efficiency of $120 \%$ with the overall resolution showing little degradation over that of the worst detector.

42. C. E. Moss, W. Bernard, E. J. Dowdy, C. Garcia, M. C. Lucas, and J. C. Pratt, "An Array of Germanium Detectors for Nuclear Safeguards," in "The Proceedings of the INMM 24th Annual Meeting," Vail, Colorado, July 10-13, 1983, Vol. XII, pp. 129-132.

Our gamma-ray spectrometer system, designed for field use, offers high efficiency and high resolution for safeguards applications. The system consists of three $40 \%$ high-purity germanium detectors and a LeCroy 
3500 data acquisition system that calculates a composite spectrum for the three detectors. The LeCroy 3500 mainframe can be operated remotely from the detector array with control exercised through modems and the telephone system. System performance with a mixed source of ${ }^{125} \mathrm{Sb},{ }^{154} \mathrm{Eu}$, and ${ }^{155} \mathrm{Eu}$ confirms the expected efficiency of $120 \%$ with an overall resolution that is between the resolution of the best detector and that of the worst.

43. C. E. Moss, E. J. Dowdy, A. E. Evans, M. E. Hamm, M. C. Lucas, and E. R. Shunk, "A Measurement System for Nuclear Safeguards Based on BismuthGermanate Scintillators," in "The Proceedings of the ESARDA Fifth Annual Symposium on Safeguards and Nuclear Material Management," Versailles, France, April 19-21, 1983, pp. 297-299.

To determine gamma-ray flux spectra, with high efficiency as the foremost objective, we constructed a system that uses bismuth-germanate scintillators as sensors. The system, consisting of eight scintillators and a LeCroy 3500 data acquisition system, has been calibrated and characterized from 0.06 to $8.29 \mathrm{MeV}$. By fitting the calibration spectra with a function containing 17 parameters, we were able to construct theoretical response functions, which we then used to obtain the gamma-ray flux spectra at multiple space points resulting from a variety of radioactive objects of interest in nuclear safeguards. The results of our procedure agree with calculated values to within less than $10 \%$.

44. N. Nicholson, J. D. Atencio, T. H. Kuckertz, and C. D. Ethridge, "A Real-Time Inventory System for Special Nuclear Material in Storage," Los Alamos National Laboratory report LA-9824-MS (June 1983).

Special nuclear material (SNM) in long-term storage is particularly vulnerable to diversion, hence it must be inventoried periodically. Inventory procedures are time consuming and expose personnel unnecessarily to radiation. Los Alamos scientists have developed an alternative method for taking inventory: a system that provides continuous surveillance of all SNM stored in a vault. The system features a network of shelf monitors, one monitor for each container in storage. A shelf monitor detects the gamma-ray emissions and weight of the container resting on it and transmits the data in real time to a minicomputer for analysis. Reliable and inexpensive, the shelf monitors are attractive for deployment in a storage facility where hundreds or thousands of them are required.

45. C. A. Ostenak and A. F. Cermak "Comparison of Predicted and Measured Pulsed-Column Profiles and Inventories," presented at "The ANS/INMM Conference on Safeguards Technology: The Pro-
cess-Safeguards Interface," Hilton Head Island, South Carolina, November 28-December 2, 1983 (to be published).

Nuclear materials accounting and process control in fuels reprocessing plants can be improved by nearreal-time estimation of the in-process inventory in solvent-extraction contactors. Experimental studies were conducted on pilot- and plant-scale pulsed columns by Allied-General Nuclear Services (AGNS), and the extensive uranium concentration-profile and inventory data were analyzed by Los Alamos and AGNS to develop and evaluate different predictive inventory techniques. Preliminary comparisons of predicted and measured pulsed-column profiles and inventories show promise for using these predictive techniques to improve nuclear materials accounting and process control in fuels reprocessing piants.

46. G. Papcun, W. Ford, and A. R. Davis, "Signal Detection and Classification Analyses of Voice Input Systems," presented at "The 1983 Voice Data Entry Systems Application Conference," Chicago, Illinois, September 27-29, 1983.

Decision tasks may be classified as recognition tasks, classification tasks, or as a combination of the two. In this paper we present an extension to the theories of detection and classification so that composite tasks are also included. We show that these differing tasks require differing techniques for their analysis, and we show how to use appropriate analyses to make them commeasurable. Detection tasks, such as talker verification, can be evaluated independently of threshold by the measures $d^{\prime}$ or $P(A)$. These measures can also be calculated for classification tasks such as word recognition, but the number of response alternatives, the distributions of their scores, and their intercorrelations must be taken into account. Composite detection and classification tasks, such as word recognition with a rejection threshold, can also be treated, but assumptions must be made or information gathered about the distributions underlying their behavior. Computer programs are provided for analyzing and interpreting data for composite detection and classification tasks, which are of particular importance because they are the basis for most practical computer voice input systems and they are also the tasks involved in ordinary human perception. The analyses discussed herein are applicable to computers and to other detection and classification systems such as people.

47. J. L. Parker, "Are 0.1\%-Accurate Gamma-Ray Assays Possible for ${ }^{235} \mathrm{U}$ Solutions?" in "The ANS/INMM Conference on Safeguards Technology: The Process-Safeguards Interface," Hilton Head Island, South Carolina, November 28-December 2, 1983 (to be published). 
The factors influencing the accuracy of passive gamma-ray assay of uniform, homogeneous solution sainples have been studied in some detail, particularly for the assay of ${ }^{235} \mathrm{U}$ in uranium solutions. Factors considered are the overall long-term electronic stability, the information losses caused by the rate-related electronic processes of pulse pileup and deadtime, and the self-attenuation of gamma rays within the samples. Both experimental and computational studies indicate that gamma-ray assay procedures for solution samples of moderate size (from $\sim 10$ to perhaps a few hundred milliliters) are now capable of accuracies approaching $0.1 \%$ in many practical cases.

48. J. R. Phillips and G. E. Bosler, "Calculated Response Contributions of Gamma Rays Emitted from Fuel Pins in an Irradiated PWR Fuel Assembly," Los Alamos National Laboratory report LA-9837-MS (August 1983).

The relative contributions of gamma rays originating in specific fuel pins to the total gamma-ray source strength were calculated as a function of initial gamma-ray energy for a pressurized-water-reactor fuel assembly. Gamma-ray energies used in the calculations ranged from 511 to $21 \mathrm{~s} 5 \mathrm{keV}$, the energy range in which most long-lived fission and activation products emit gamma rays. Nondestructive gamma-ray measurement techniques use specific signatures of ${ }^{137} \mathrm{Cs}$ or ${ }^{134} \mathrm{Cs} /{ }^{137} \mathrm{Cs}$ and ${ }^{154} \mathrm{Eu} /{ }^{137} \mathrm{Cs}$ isotopic ratios to determine the exposures of irradiated fuel assemblies. Nearly $92 \%$ of the measured ${ }^{137} \mathrm{Cs}$ signature (661.6 $\mathrm{keV}$ ) originates in the three outer rows of fuel pins. However, because only $78 \%$ of the ${ }^{154} \mathrm{E} u$ signature $(1275 \mathrm{keV}$ ) originates in these three outer rows, a ratio of ${ }^{154} \mathrm{Eu} /{ }^{137} \mathrm{Cs}$ does not sample exactly the same volume segment of the fuel assembly. Other examples are presented that evaluate the effect of using a collimator at different angular orientations on quantifying the contributions from specific fuel pins to the total source strength.

49. J. R. Phillips, G. E. Bosler, J. K. Halbig, S. F. Klosterbuer, H. O. Menlove, and P. M. Rinard, "Experience Using a Spent-Fuel Measurement System," in "The Proceedings of the INMM 24th Annual Meeting on Nuclear Materials Management," Vail, Colorado, July 10-13, 1983, Vol. XII, pp. 175-181.

A portable nondestructive measurement system has been used to measure neutron and gross gamma-ray emission from irradiated BWR and PWR fuel assemblies. The system consists of two principal components: a water-tight polyethylene detector head containing two sets of ion chambers and fission chambers for measuring simultaneously opposite sides of the fuel assembly, and a battery-powered electronics unit with a microprocessor for performing internal diagnostics and for assisting the operator in the collec- tion and analysis of the data. We have used the system to measure $52 \mathrm{BWR}$ and 19 PWR fuel assemblies in reactor spent-fuel storage pools. An average time of five minutes was required to (1) position the fuelhandling bridge, (2) vertically raise the fuel assembly, (3) perform the measurements, and (4) replace the fuel assembly in the storage rack. The neutron results with proper calibration can be used to determine the exposures of individual fuel assemblies with an average zccuracy of approximately 5\%. Consistency of operator-declared values for cooling time and exposure can be verified using the gross gamma-ray measurements with an average accuracy of about $10 \%$. Fuel assemblies with unusual irradiation histories or reconstituted fuel pins were easily identifiable.

50. J. R. Phillips, J. K. Halbig, P. M. Rinard, and G. E. Bosler, "NDA Measurement of the Demineralizers at TMI-2," Los Alamos National Laboratory report LA-9795-MS (August 1983).

Nondestructive measurements were performed to estimate the amount of fuel material deposited in the two demineralizers during the accident at TMI-2. Gross gamma-ray measurements were used to characterize the horizontal and vertical activity profiles of gamma-emitting fission products in Demineralizer A. A Be( $\gamma, n)$ detector, sensitive to gamma rays with energies above $1.667 \mathrm{MeV}$, was used to estimate the quantity of ${ }^{144} \mathrm{Ce}-{ }^{144} \mathrm{Pr}$ present, which was assumed to be directly proportional to the amount of fuel prcsent. A variety of source distributions was modeled using the Los Alamos Monte Carlo transport code to calculate the fraction of gamma rays that would strike the detector with energies above the $1.667-\mathrm{MeV}$ threshold. The estimated amount of uranium depends on the assumed source distribution and material density as well as on the specific orientation of the detector. We estimate an upper limit to be in the range of $7 \mathrm{~kg}$ and a lower limit to be $2 \mathrm{~kg}$ for Demineralizer A. Demineralizer B is estimated to contain approximately $0.7 \mathrm{~kg}$ of uranium.

51. R. R. Picard and R. S. Marshall, "Uranium Holdup Modeling," Los Alamos National Laboratory report LA-9853-MS (November 1983).

Statistical modeling of nuclear materials holdup in processing facilities can play an important role in materials accounting. Detailed knowledge of process operation, variability of process conditions, quality of measurements, and measurement standards impact the value of model-based estimates. Recognition of both the benefits and the limitations of model-based estimates and the periodic updating of such estimates are essential to maintaining a credible holdup estimation model.

52. K. K. S. Pillay, "Use of Tracers in Materials Holdup Study," in "The Proceedings of the INMM 24th 
Annual Meeting on Nuclear Materials Management," Vail, Colorado, July 10-13, 1983, Vol. XII, pp. 182-186.

Holdup measurements of special nuclear materials in large processing facilities offer considerable challenges to conventional nondestructive assay techniques. The use of judiciously chosen radioactive tracers offer a unique method of overcoming this difficulty. Three examples involving the use of ${ }^{46} \mathrm{Sc}$ and fission products from activated uranium in large-scale experimental studies of uranium holdup are discussed. $A$ justification for the method and its advantages along with examples of successful applications of this technique for large-scale experimental studies are presented.

53. P. Rinard, "A Spent-Fuel Cooling Curve for Safeguard Applications of Gross-Gamma Measurements," Los Alamos National Laboratory report LA-9757-MS (ISPO-195) (April 1983).

Gross-gamma detectors can be used to gather data from spent-fuel assemblies in a simple and rapid manner. Using these data, inspectors can generate a power-law curve to check the consistency of the declared values with the measured values; points outside the curve indicate erroneously declared values or removal of material. Simple types of erroneously declared values can be detected immediately, whereas subtle types may require a second measurement and more subtle types may escape detection. If measurements of passive emissions of neutrons from the assemblies are made in addition to the gamma measurements, the values of the exposures and cooling times can be estimated independent of the operator-declared values. Although not yet demonstrated, it may be possible to obtain crude estimates of the exposures and cooling times from the gamma measurements alone.

54. P. A. Russo, R. B. Strittmatter, E. L. Sandford, I. W. Jeter, E. McCullough, and G. L. Bowers, "Operation of Automated NDA Instruments for In-Line HEU Accounting at Y-12," in "The ANS/INMM Conference on Safeguards Technology: The Process-Safeguards Interface," Hilton Head Island, South Carolina, November 28-December 2, 1983 (to be published).

Two automated nondestructive assay instruments developed at Los Alamos in support of nuclear materials accounting needs are currently operating in-line at the Y-12 Plant for recovery of highly enriched uranium. One instrument provides the HEU inventory in the secondary solvent extraction system, and the other monitors HEU concentration in the secondary intermediate evaporator. Both instruments were installed in December 1982. Operational evaluation of these instruments has been a joint effort of Y-12 and Los Alamos. This has included comparison of the solvent extraction system inventories with direct measurement performed on the dumped solution components of the solvent extraction system, as well as comparisons of concentration assay results with the external assays of samples withdrawn from the process. The function, design, and preliminary results of the operational evaluation are reported.

55. T. E. Sampson and R. Gunnink, "The Propagat!on of Errors in the Measurement of Plutonium Isotopic Composition by Gamma-Ray Spectroscopy," Los Alamos National Laboratory document LA-UR-83-1520 (to be published in INMM journal).

We discuss methods for the propagation of errors in the determination of plutonium isotopic composition and specific power by gamma-ray spectroscopy techniques. The 1975 ANSI calorimetry standard is shown to be in error in this regard. The developed formulas are compared with measurements for applicable cases. Some examples of the sensitivity of the specific power to measurement biases are presented.

56. T. E. Sampson, "In-Plant Experience With Automated Gamma-Ray Spectroscopy Systems for Plutonium Isotopic Composition Measurements," in "The ANS/INMM Conference on Safeguards Technology: The Process-Safeguards Interface," Hilton Head Island, South Carolina, November 28-December 2, 1983 (to be published).

Two gamma-ray spectroscopy systems for the nondestructive measurement of the isotopic composition of plutonium are described. One system is at the Savannah River Plant and the other is at the Los Alamos Plutonium Facility. Results from a year's operation of the Los Alamos system are presented.

57. T. E. Sampson, E. L. Sandford, D. F. Bowersox, S. S. Johnson, S. -T. Hsue, J. L. Parker, K. Kroncke, and G. Walton, "In-Plant Experience with Automated Gamma-Ray Spectroscopy Systems for Plutonium Isotopic Composition," Los Alamos National Laboratory report LA-9789-MS (August 1983).

Two gamma-ray spectroscopy systems for the nondestructive measurement of the isotopic composition of plutonium are described. One system is at the Savannah River Plant and the other is at the Los Alamos Plutonium Facility. Results from a year's operation of the Los Alamos system are presented.

58. R. Sher, “Active Neutron Coincidence Counting for the Assay of MTR Fuel Elements," Los Alamos National Laboratory report LA-9665-MS (ISPO-186) (February 1983).

The Active Well Coincidence Counter (AWCC) and the neutron coincidence collar (CC) were investigated 
for their suitability to assay materials testing reactor (MTR) fuel elements. The AWCC was used with its special insert to hold the fuel element and interrogation source. The $\mathrm{CC}$ was modified by the addition of polyethylene liners $2.5 \mathrm{~cm}$ ( 1 in.) thick on the sides. For a typical MTR element $\left(\sim 220 \mathrm{~g}{ }^{235} \mathrm{U}\right.$ and $1000-\mathrm{s}$ count times), statistical errors were $\sim 1.6 \%$ for the $\mathrm{CC}$ and $\sim 0.6 \%$ for $A W C C$. For either instrument, the change in count rate corresponding to the removal or addition of one fuel plate (with an 18-plate element) was $\sim 3.8 \%$; thus, either instrument can detect removal of one pizte. The AWCC can also detect removal of one plate in count times that are considerably less than $1000 \mathrm{~s}$. Various functions were investigated to fit the coincidence count rate vs ${ }^{235} \mathrm{U}$ mass curve for the AWCC. Programs have been written for the Hewlett-Packard HP-97 calculator to calculate the calibration constants of these functions by a least squares technique. Coincidence count rates in the AWCC depend on the orientation of the plates of the fuel elements because of the counting efficiency variation in the insert. To lessen this dependence, the MTR element should be counted with its plates positioned vertically, that is, parallel to the radius of the device. For the collar, the effect of plate orientation is much smaller.

59. E. R. Shunk, J. D. Atencio, H. F. Atwater, W. Bernard, J. M. Bieri, J. T. Caldwell, S. W. France, R. D. Hastings, G. C. Herrera, and W. E. Kunz, "Assay System to Measure Crate-Size Bulk Transuranic Waste," Proceedings of the INMM 24th Annual Meeting on Nuclear Materials Management, Vail, Colorado, July 10-13, 1983, Vol. XII, pp. 80-84.

The Advanced Nuclear Technology Group of the Los Alamos National Laboratory recently designed and built a combined passive- and active-neutron assay system on which group members performed initial characterization measurements. The system is intended to provide sensitive assays at the $100-\mathrm{nCi} / \mathrm{g}$ level of both spontaneous fission and fissile transuranic isotopes in large crates of bulk waste. Such crates can be accommodated in the system's assay chamber whose internal dimensions are 64 by 64 by $96 \mathrm{in}$. Moderated ${ }^{3} \mathrm{He}$ proportional counters provide a measured total $4 \pi$ neutron detection efficiency of 13.5 $\pm 1 \%$ for bare- and moderated-fission spectrum sources placed anywhere within the volume of a standard empty crate. Initial passive neutron coincidence. measurements indicate ${ }^{240} \mathrm{Pu}$ detection sensitivity of about $10 \mathrm{mg}$. Enhancement of the fissile-isotope assay sensitivity results from lining the assay chamber walls with a thick layer of graphite and placing specially designed prompt-fission neutron detectors within the walls. Initial pulsed-neutron measurements indicate a fissile assay sensitivity of about $30 \mathrm{mg}$ of ${ }^{239} \mathrm{Pu}$. After completion of checkout and initial calibration measurements at Los Alamos, the assay system will be installed at a DOE facility for a 1-year test-and-evalua- tion program beginning in July 1983. During the period, actual TRU waste crates averaging nearly $1000 \mathrm{~kg}$ each will be assayed on a routine basis.

60. J. K. Sprinkle, Jr., "An End-Crop Box Counter," in "The ANS/INMM Conference on Safeguards Technology: The Process-Safeguards Interface," Hilton Head Island, South Carolina, November 28-December 2, 1983 (to be published).

A nondestructive assay instrument that measures large quantities of low-enriched uranium metal is described. The assay is based on the spontaneous totals neutron signal.

61. J. K. Sprinkle, R. N. Likes, J. L. Parker, and H. A. Smith, "Reference Materials for Nondestructive Assay of Special Nuclear Material - Volume 1: Uranium Oxide Plus Graphite Powder," Los Alamos National Laboratory report LA-9910-MS, Vol. 1, NUREG/CR-3522, Vol. I (October 1983).

This manual describes the fabrication of reference materials for use in gamma-ray-based no.idestructive assay of low-density uranium-bearing samples. The sample containers are $2-\ell$ bottles. The reference materials consist of small amounts of $\mathrm{UO}_{2}$ spread throughout a graphite matrix. The ${ }^{235} \mathrm{U}$ content ranges from 0 to $100 \mathrm{~g}$. The manual also describes the farfield assay procedure used with low-resolution detectors.

62. J. K. Sprinkle, R. N. Likes, J. L. Parker, and H. A. Smith, "Reference Materiais for Nondestructive Assay of Special Nuclear Material - Volume 2: Thin Metal Foils of Highly Enriched Uranium," Los Alamos National Laboratory report LA-9910-MS, Vol. 2, NUREG/CR-3522, Vol. 2 (October 1983).

This manual describes the fabrication of reference materials for use in gamma-ray-based nondestructive assay of small high-density uranium samples. The sample containers are small Petri dishes. The reference materials consist of thin circular discs of highly enriched uranium metal foil. The ${ }^{235} \mathrm{U}$ content ranges from 0.2 to $10 \mathrm{~g}$. The manual also describes the assay procedure used with low-resolution detectors.

63. J. K. Sprinkle, Jr., and M. M. Stephens, "End-Crop Box Counter Manual," Los Alamos National Laboratory report LA-9781-M (June 1983).

The end-crop box counter was designed at Los Alamos National Laboratory for assaying filled shipping boxes of end crops from the fuel extrusion process used in fuel rod fabrication at United Nuclear Corporation. This manual details the measurement technique and the hardware, software, and calibration. It also provides instructions for operation and troubleshooting. The section on operation can be used as a separate operations manual by the routine user. 
64. D. Stirpe, A. S. Goldman, and R. G. Gutmacher, "MC\&A Reform Amendment: Example System Beta Variance Estimates and Performance Evaluation," Los Alamos National Laboratory report LA-9708-MS, NUREG/CR-3221 (May 1983).

Variance estimates and tests for compliance with the loss-detection provisions of the proposed MC\&A Reform Amendment are given for each of the materials control and accounting (MC\&A) systems of the three process lines that make up Example System Beta. These lines are (1) $\mathrm{UF}_{6}$-to-oxide conversion, (2) hightemperature gas reactor (HTGR) coated-particle fabrication, and (3) uranium scrap recovery. For each material control unit (MCU) in the MC\&A systems for these lines, we identify measurement poinis and appropriate measurement techniques; we present the materials balance and variance equations; we calculate the associated standard deviations; and finally, we test the MC\&A systems for compliance with the Reform Amendment loss-detection requirements. Wherever potential problems in satisfying the requirements of the MC\&A Reform Amendment are recognized, we suggest alternative $\mathrm{MCU}$ structures that incorporate additional measurement capability or modifications to the process. With appropriate process/ instrumentation modifications of the conversion and scrap recovery processes, the prompt loss-detection provisions of the Pefurm Amendment can be satisfied without imposing administrative controls. Levels of performance against trend and cumulative loss requirements are also assessed. The reference HTGR coated-particle process satisfies some of these provisions if administrative controls are imposed.

65. J. E. Stewart, "Neutron Production by Alpha Particles in Thin Uranium Hexafluoride," Los Alamos National Laboratory report LA-9838-MS (July 1983).

Alpha-particle-induced neutrons from $\mathrm{UF}_{6}$ serve as an indicator of ${ }^{235} \mathrm{U}$ enrichment and may be exploited for safeguards purposes. If the $U F_{6}$ density is low enough, neutron production is reduced as a result of alpha-particle escape before $(a, n)$ reactions with ${ }^{19} \mathrm{~F}$. Calculational methods and results are presented that enable prediction of neutron production in low-density ("thin") $U_{6}$ as encountered in the gas centrifugation method of uranium enrichment. Neutron production is shown to be strongly dependent on average $\mathrm{UF}_{6}$ density and weakly dependent on rotational speed in an operating centrifuge.

66. J. E. Stewart and H. O. Menlove, "Direct Fissile Assay of Highly Enriched UF $_{6}$ Using Random SelfInterrogation and Neutron Coincidence Response," in "The ANS/INMM Conference on Safeguards Technology: The Process-Safeguards Interface," Hilton Head Island, South Carolina, November 28December 2, 1983 (to be published).
A new nondestructive method for direct assay of ${ }^{235} \mathrm{U}$ mass contained in Model 5A uranium hexafluoride $\left(U F_{6}\right)$ product storage cylinders has been successfully tested in the laboratory and under field conditions. The technique employs passive neutron self-interrogation and uses the ratio of coincidences-to-totals counts as a measure of bulk fissile mass. The accuracy of the method is $6.8 \%(1 \sigma)$ based on field measurements of 44 Model SA cylinders, 11 of which were either only partially filled or contained reactor return material. The cylinders contained $U_{6} F_{6}$ with enrichments from 5.96\% to 97.6\%. Count times were 3-6 min depending on ${ }^{235} \mathrm{U}$ mass. Samples ranged from below $1 \mathrm{~kg}$ to over $16 \mathrm{~kg}$ of ${ }^{235} \mathrm{U}$. Because the method relies primarily on fast neutron self-interrogation, complete sampling of the $\mathrm{UF}_{6}$ takes place. This feature alleviates inhomogeneity problems and offers increased assurance of the presence of stated amounts of bulk fissile material as compared with current verification methods.

67. R. B. Strittmatter, L. A. Stovall, and J. K. Sprinkle, "Development of an Enrichment Monitor for the Portsmouth GCEP," in "The ANS/INMM Conference on Safeguards Technology: The ProcessSafeguards Interface," Hilton Head Island, South Carolina, November 28-December 2, 1983 (to be published).

We have developed a gas-phase $U_{6}$ enrichment monitor for use by the International Atomic Energy Agency at the Portsmouth Gas Centrifuge Enrichment Plant. The enrichment monitoring system provides a method for effective nuclear materials accountability verification while reducing the effort for both the facility operator and the inspector. The experience with an in-plant prototype monitor, the facility and operational constraints, and the constraints related to international safeguards inspection are described in lerms of the impact on the monitor design.

68. J. Swansen and P. Collinsworth, "High-Level Neutron Coincidence Counter Maintenance Manual," Los Alamos National Laboratory report LA-9662M (ISPO-168) (May 1983).

High-Level Neutron Coincidence Counter operational (field) calibration and usage is well known. This manual makes explicit basic (shop) check-out, calibration, and testing of new units and is a guide for repair of failed in-service units. Operational criteria for the major electronic functions are detailed, as are adjustments and calibration procedures, and recurrent mechanical/electromechanical problems are addressed. Some systen tests are included for quality assurance. Data on nonstandard large-scale integrated (circuit) components and a schematic set are also included. 
69. J. W. Tape, R. B. Strittmatter, and A. L. Baker, "The Process-(International) Safeguards Interface at the Portsmouth GCEP From the Safeguards Technology Developer's Viewpoint," presented at "The ANS/INMM Conference on Safeguards Technology: The Process-Safeguards Interface," Hilton Head Island, South Carolina, November 28-December 2, 1983 (10 be published).

Tie design of a major portion of a safeguards system for possible use by the International Atomic Energy Agency (IAEA) at the Portsmouth Gas Centrifuge
Enrichment Plant is described from the viewpoint of the safeguards technology developer. The processsafeguards interface is reviewed, including safeguards, process, and operational constraints on the safeguards system. The conflicting requirements of the IAEA and the plant operator are minimized through the use of advanced technology that allows safeguards data to be acquired, analyzed, and reported in an automated, unattended mode. The proposed safeguards system will reduce the inpact of the inspections on both the operator and the IAEA. 


\section{REFERENCES}

I. S.-T. Hsue, T. E. Sampson, J. L. Parker, S. S. Johnson, and D. F. Bowersox, "Plutonium Isotopic Composition by Gamma-Ray Spectroscopy," Los Alamos Scientific Laboratory report LA-8603-MS (November 1980).

2. T. E. Sampson, S.-T. Hsue, J. L. Parker, S. S. Johnson, and D. F. Bowersox, "The Determination of Plutonium Isotopic Composition by Gamma-Ray Spectroscopy," Nuclear Instruments and Methods 193, 177 (1982).

3. T. E. Sampson, S.-T. Hsue, E. L. Sandford, J. L. Parker, D. F. Bowersox, K. Kronke, S. S. Johnson, and G. Walton, "In-Plant Experience with Automated Gamma-Ray Spectroscopy Systems for Plutonium Isotopic Composition Measurement," Los Alamos National Laboratory report LA-9789-MS (August 1983).

4. T. E. Sampson, "In-Plant Experience with Automated Gamma-Ray Spectroscopic Systems for Plutonium Isotopic Composition Measurements," Transactions of the American Nuclear Society 45, Supp 1, 17 (1983).

5. S.-T. Hsue, F. Hsue, and D. F. Bowersox, "Assay of Low-Level Plutonium Effluents," Journal of the Institute for Nuclear Materials Management X, 453 (1981).

6. N. Ensslin, E. Adams, D. F. Bowersox and J. Stewart, "Neutron Coincidence Counting of Plutonium Solutions." Transactions of the American Nuclear Society $39335(1981)$.

7. "Progress Report on FAST," :inerican Nuclear Society Nuclear News (July 1982), p.44.

8. H. O. Menlove, G. W. Eccleston, D. A. Close, and L. G. Speir, "Neutron Interrogator Assay System for the Idaho Chemical Processing Plant Waste Canisters and Spent Fuel-Preliminary Description and Operating Procedures," Los Alamos Scientific Laboratory report LA-7250-M (May 1978).

9. G. W. Eccleston, R. G. Schrandt, J. L. MacDonald, and F. H. Cverna, "Monte Carlo Calculational Design of an NDA Instrument for the Assay of Waste
Products from High Enriched Uranium Spent Fuels," in "Measurement Technology for Safeguards and Materials Control," Proceedings of American Nuclear Society Topical Meeting, Kiawah Island, South Carolina, November 26-30, 1979, NBS Special Publication 582.

10. G. W. Eccleston, H. O. Menlove, L. Decker, and M. W. Echo, "An Assay System to Measure High Enriched Uranium Spellt Fuel and Hot Solid Waste at the Idaho FAST Facility," American Institute of Chemical Engineers Annual Meeting, Orlando, Florida, February 28, 1982.

II. G. W. Eccleston, S. S. Johnson, H. O. Menlove, T. Van Lyssel, D. Black, B. Carlson, L. Decker, and M. W. Echo, "FAST Facility Spent-Fuel and Waste Assay Instrument," in "Proceedings of the ANS/INMM Conference on Safeguards Technology: The Process Safeguards Interface," Hilton Head lsland, South Carolina, November 28-December 2, 1983 (to be published).

12. P. E. Fehlau, "Vehicle SNM Monitors: Results of an Evaluation," in "Proceedings of the INMM 24th Annual Meeting on Nuclear Materials Management," Vail, Colorado, July 10-13. 1983, Vol. XII, pp. 294-300.

13. P. E. Fehlau, J. C. Pratt, J. T. Markin, and T. Scurry, Jr., "Smarter Radiation Monitors for Safeguards and Security," in "Proceedings of the INMM 24th Annual Meeting on Nuclear Materials Management," Vail, Colorado. July 10-13, 1983, Vol. XII, pp. 122-128.

14. P. A. Russo and R. B. Strittmatter, "On-Line Prototype NDA Instruments for Materia! Accounting and Monitoring of HEU Recovery Processes," Transactions of the American Nuclear Socicty 43, 274 (1982).

15. I. W. Jeter, S. E. Smith, H. H. Hogue, G. L. Bowers, P. A. Russo, and R. B. Strittmatter, "Applications of NDA Instrumentation in the Y-12 Highly Enriched Uranium Recovery Facility," in "Proceedings of Orlando International AlChE Meeting." Orlando, Florida (1982). 
16. P. A. Russo, R. B. Strittmatter, E. L. Sandford, I. W. Jeter, E. McCullough, and G. L. Bowers, "Operation of Automated NDA Instruments for In-Line HEU Accounting at Y-12," in "Proceedings of the ANS/INMM Conference on Safeguards Technology: The Process-Safeguards Interface," Hilton Head Island, South Carolina, November 28-December 2, 1983 (to be published).

17. R. H. Augustson, R. B. Walton, W. Harbarger, J. Hicks, G. Timmons, D. Shissler, R. Tayloe, S. Jones, R. Harris, and L. Fields, "Measurements of Uranium Holdup in an Operating Gaseous Diffusion Enrichment Plant," Los Alamos National Laboratory document LA-UR-83-3381, to be published in "Proceedings of the ANS/INMM Conference on Safeguards Technology: The ProcessSafeguards Interface," Hilton Head Island, South Carolina, November 28-December 2, 1983.

18. J. T. Caldwell, D. A. Close, T. H. Kuckertz, W. E. Kunz, J. C. Pratt, K. W. Haff, F. J. Schultz, "Test and Evaluation of a High Sensitivity Assay System for Bulk Transuranic Waste," Los Alamos National Laboratory document LA-UR-83-2084 (24th Annual Meeting of the INMM, Vail, Colorado, July 10-13, 1983).

19. E. R. Shunk, J. D. Atencio, H. F. Atwater, W. Bernard, J. M. Bieri, J. T. Caldwell, S. W. France, R. D. Hastings, G. C. Herrera, and W. E. Kunz, "Assay System to Measure Crate-Size Bulk Transuranic Waste," Los Alamos National Laboratory document LA-UR-83-1999 (24th Annual Meeting of the INMM, Vail, Colorado, July 10-13, 1983).

20. J. T. Caldwell, D. A. Close, T. W. Crane, T. H. Kuckertz, W. E. Kunz, R. Morgado, J. C. Pratt, E. R. Shunk, C. J. Umbarger, L. A. Franks, and S. M. Kocimski, "Measurement of Transuranic Content in Wastes," Nuclear and Chernical Waste Management 4, 11-17 (1983).

21. J. P. Shipley and D. B. Smith, "Safeguards and Security Status Report, August 1982-January 1983," Los Alamos National Laboratory report LA-9821-PR (September 1983).

22. J. L. Parker, "Are 0.1\%-Accurate Gamma-Ray Assays Possible for ${ }^{235} \mathrm{U}$ Solutions?," in "Proceedings of the ANS/INMM Conference on Safeguards Technology: The Process-Safeguards Interface,"
Hitton Head Island, South Carolina, November 28December 2, 1983(to be published).

23. M. P. Baker, T. Sampson, A. Baker, S. -T. Hsue, S. Johnson, and L. Speir, "A Conceptual Design for an Integrated Nondestructive Assay System at the SRP New Special Recovery Facility," Los Alamos National Laboratory, Group Q-1 document Q-1-83-328.

24. J. K. Sprinkle, Jr., and M. M. Stephens, "End-Crop Box Counter Manual," Los Alamos National Laboratory report LA-9781-M (June 1983).

25. J. K. Sprinkle, Jr., "An End-Crop Box Counter," Los Alamos National Laboratory document LAUR-83-3199 in "Proceedings of the ANS/INMM Conference on Safeguards Technology: The Process-Safeguards Interface," Hilton Head Island, South Carolina, November 28-December 2, 1983 (to be published).

26. H. Ottmar and E. Eberle, "Determination of Plutonium Isotopic Composition by Gamma Spectrometry: Results from Interlaboratory Comparison Measurements Organized by ESARDA," in Proceedings of the Ist Annual ESARDA Symposium on Safeguards and Nuclear Materials Management (European Safeguards Research and Development Association, 1979), ESARDA 10, pp. 366-373.

27. J. G. Fleissner, J. F. Lemming, and J. Y. Jarvis, "Study of a Two-Detector Method for Measuring Plutonium Isotopics," in Proccedings of the ANS Topical Conference on Measurement Technology for Safeguards and Materials Control (National Bureau of Standards, Washington, DC, 1980); T. E. Sampson, S. -T. Hsue, J. L. Parker, S. S. Johnson, and D. F. Bowersox, "The Determination of Plutonium Isotopic Composition by Gamma-Ray Spectroscopy." Nuclear Instruments and Methods 193, 117 (1982).

28. L. R. Cowder, S. -r. Hsue, S. S. Johnson, J. L. Parker, P. A. Russo, J. K. Sprinkle, Y. Asakura, T. Fukuda, and 1. Kondo, "Gamma-Ray NDA Assay System for Total Plutonium and Isotopics in Plutonium Product Solutions," in Proceedings of ANS Topical Conference on Measurement Technology for Safeguards and Matcrials Control (National Bureau of Standards, Washington, DC, 1980); R. Gunnink, A. L. Prindle, J. B. Niday, A. L. VanLehn, and 
Y. Asakura, "TASTEX Gamma Spectrometer System for Measuring Isotopic and Total Plutonium Concentrations in Solutions," in Journal Institute Nuclear Materials Management VIII, 429 (1979).

29. T. K. Li, T. E. Sampson, and S. S. Johnson, "Plutonium Isotopic Measurement for Small Product Samples," in Proceedings of the ESARDA Fifth Annual Symposium on Safeguards and Nuclear Material Management, Versailles, France, April 19-21, 1983 (ESARDA 1983), ESARDA 16, pp. 289-291.

30. T. K. Li, "Determination of Plutonium Isotopic Ratios by Using Low-Energy Gamma-Ray Spectroscopy," in "Proceedings of the ANS/INMM Topical Conference on Safeguards Technology: The Process-Safeguards Interface," Hilton Head Island, South Carolina, November 28-December 2, 1983 (to be published).

31. W. J. McGonnagle, M. K. Holland, C. S. Reynolds, N. M. Trahey, and A. C. Zook, "Evaluation and Calibration of a Los Alamos National Laboratory

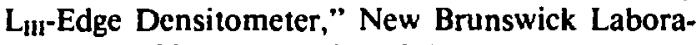
tory report NBL-307 (July 1983).

32. D. G. Langner, J. K. Sprinkle, Jr., and P. A. Russo, "Calibration Response of the $\mathrm{L}_{\mathrm{mI}}$-Edge Densitometer," in "Saieguards and Security Status Report, February-July 1982," Los Alamos National Laboratory report LA-9595-PR (February 1983).

33. R. B. Walton, T. D. Reilly, J. L. Parker, J. H. Menzel, E. D. Marshall, and L. W. Fields, "Measurements of Uraniun Hexafluoride Cylinders with Portable Instruments," Nuclear Technology' 21, 133-148 (1974).

34. H. O. Menlove, "Description and Performance Characteristics for the Neutron Coincidence Collar for the Verification of Reactor Fuel Assemblies," Los Alamos National Laboratory report LA-8939. MS (ISPO-142) (Augusi 1981).

35. H. O. Menlove, "Description and Operation Manual for the Active Well Coincidence Counter," Los Alamos National Laboratory manual LA-7823-M (May 1979).

36. N. Ensslin, A. Gibbs, D. Denard, and P. Deason, "Test and Evaluation of the Dual-Range Coincidence Counter at the Savannah River Plant," Los
Alamos National Laboratory report LA-8803-MS (April 1981).

37. M. S. Krick and H. O. Menlove, "The High-Level Neutron Coincidence Counter (HLNCC) Users" Manual," Los Alamos National Laboratory manual LA-7779-M (ISPO-53) (June 1979).

38. D. D. Cobb, J. R. Phillips, G. E. Bosler, G. W. Eccleston, J. K. Halbig, C. R. Hatcher, and S. -T. Hsue, "Nondestructive Verification and Assay Systems for Spent Fuels," Los Alamos National Lahoratory report LA-9041, Vol. I (April 1982).

39. D. D. Cobb, J. R. Phillips, M. P. Baker, G. E. Bosler, G. W. Eccleston, J. K. Halbig, S. L. Klein, S. F. Klosterbuer, H. O. Menlove, C. A. Ostenak, and C. C. Thomas, Jr., "Nondestructive Verification and Assay Systems for Spent Fuels: Technical Appendixes," Los Alamos National Laboratorv report LALA-9041, Vol. II (April 1982).

40. K. V. Nixon and C. Garcia, Jr., "Hand-Held PulseTrain-Analysis Instrument," IEEE Transactions in Nuclear Science NS-30, 331 (1983).

41. P. E. Fehlau, "Gamma-Ray Detectors for Intelligent, Hand-Held Radiation Monitors," in Proceedings of IEEE 1983 Nuclear Science Symposium, San Francisco, California, Octooer 19-21, 1983.

42. P. E. Fehlau, "Quantitative Radiation Monitors for Containment and Surveillance." in Procecdings of the ESARDA Figh Ammual Symposium on Safeguards and Nuclear Materials Management, Versailles, France, April 19-21, 1983, (ESARDA 1983) ESARDA 16, pp. 153-157.

43. S. F. Marsh, M. R. Betts, and J. E. Rein, "Determination of Submicromolar Amounts of Uranium (VI) by Complexometric Titration with Pyridine-2, 6-Dicarboxylic Acid." Analytica Chimica Acta 119, 401-404 (1980).

44. J. W. Tape, R. B. Strittmatter, and A. L. Baker, "The Process (International) Safeguards Interface at the Portsmouth GCEP from the Safeguards Technology Developer's Viewpoint." in "Proceedings of the ANS/INMM Conference on Safeguards Technology: The Process-Safeguards Interface," Hilten Head Island, South Carolina, November 28-December 2, 1983 (to be published). 
45. R. G. Cardwell, J. M. Younkin, and D. W. Swindle, Jr., "Gas-Phase Sampling of $U F_{6}$ Cylinders in International Safeguards," in "Proceedings of the ANS/INMM Conference on Safeguards Technology: The Process-Safeguards Interface," Hitton Head lsland, Soüth: Carolina, Novèmber 28-December $\dot{2}, 1983^{\circ}$ (to be püblished).

46. A. Fainberg and R. Mitchell, "- A Portable LoadCell-Based System for Weighing UF 6 Cylinders," in "Proceedings of the ANS/INMM Conference on Safeguards Technology: The Process-Safeguards Interface," Hilton Head Island, South Carolina, November 28-December 2, 1983 (to be published).

47. R. B. Strittmatter, "A Gas-Phase UF ${ }_{6}$ Enrichment Monitor," Nuclear Technology 59, 355 (1982).

48. R. B. Strittmatter, L. A. Stovall, and J. K. Sprinkle, "Development of An Enrichment Monitor for the Portsmouth GCEP," in "Proceedings of the ANS/INMM Conference on Safeguards Technology: The Process-Safeguards Interface," Hilton Head Island, South Carolina, November 28-December 2,1983 (to be published).

49. A. L. Baker, P. W. Harris, and W. C. Fienning, "Computer Safeguards System for the IAEA Inspectors at GCEP," in "Proceedings of the ANS/INMM Conference on Safeguards Technology: The Process-Safeguards Interface," Hilton Head Island, Eouth Carolina, November 28-December 2, 1983 (to be published).

50. J. E. Stewart, S. C. Bourtet, R. W. Slice, J. T. Markin, C. A. Coulter, and C. A. Spirio, “An Area Neutron-Monitoring Strategy for International Inspections at Centrifuge Enrichment Plants," in "Safeguards and Security Status Report, FebruaryJuly 1982," J. P. Shipley and D. B. Smith, Compilers, Los Alamos National Laboratory report LA-9595-PR, (February 1983).

51. J. T. Markin, J. E. Stewart, and A. S. Goldman, "Data Analysis for Neutron Monitoring in an Enrichment Facility," presented at the 4th Annual ESARDA Symposium on Harmonization and Standardization, Petten, the Netherlands, April 27-29, 1982 (to be published).
52. H. O. Menlove, O. R. Holbrooks, and A. Ramalho, "Inventory Sample Coincidence Counter Manual," Los Alamos National Laboratory report LA-9544M (ISPO-181) (November 1982).

53. J. E. Swansen, P. R. Collinsworth, and M. S. Krick, "Shift-Register Coincidence Electronics System for Thermal Neutron Counters," Los Alamos Scientific Laboratory report LA-8319-MS (April 1980); Nuclear Instruments and Methods 176 (1980).

54. R. H. Allardice, G. Bailey, W Brenmen, S. Hackney, E. Lillyman, O, Pugh, and J. Reekie, "Management of Radioactive Solid Waste Arisings from PFR Reprocessing," Fast Reactor Fuel Cycles, ICE, London, 1981, PWMWG paper 77.

55. G. W. Eccleston, H. O. Menlove, L. Decker, and M. W. Echo, "An Assay System to Measure HighEnriched Uranium spent Fuel and Hot solid Waste at the Idaho FAST Facility," American Institute of Chemical Engineers Annual Meeting, Orlande. Florida, February 28, 1982.

56. J. T. Markin, C. A. Coulter, R. G. Gutmacher, and W. J. Whitty, "Optimizing the Design of International Safeguards Inspection Systems," Proceedings of the ESARDA Fifth Annual Symposium on Safeguards and Nuclear Materials Managemen, Versailles, France, April 19-21, 1983 (ESARDA 1983), ESARDA 16, pp. 51-55.

57. J. K. Sprinkle, R. N. Likes, J. L. Parker, and H. A. Smith, "Reference Materials for Nondestructive Assay of Special Nuclear Matcrial, Vol. 1: Uranium Oxide Plus Graphite Powder," Los Alamos National Laboratory report LA-9910-MS, NUREG/CR-3522 (October 1983).

58. J. K. Sprinkle, R. N. Likes, and H. A. Smith, "Reference Materials for Nondestructive Assay of Special Nuclear Material, Vol. 2: Thin Metal Foils of Highly Enriched Uranium," Los Alamos National Laboratory report LA-9910-MS, NUREG/CR-3522 (October 1983). 